Supplementary material for

\title{
A New Convergent Strategy for the Synthesis of Calixarenes via a Triple Annulation reaction of Fischer Carbene Complexes
}

Vijayagopal Gopalsamuthiram and William D.Wulff

Department of Chemistry, Michigan State University, East Lansing, MI 48824

Contents:

1. Experimental Procedures and Spectral Data for All New Compounds S-2 - S-30.

2. ${ }^{1} \mathrm{H}$ NMR Spectra for Calixarenes $\mathbf{3 a} \mathbf{-} \mathbf{3 i} . \quad$ S-31-S-41

3. X-Ray Structural Data for 3a-I, 3a-II and 3c. S-42-S-73 


\section{General Experimental}

All reactions were run using either oven-dried or flame-dried glassware under an inert atmosphere of argon. Chemicals used were reagent grade and used as supplied except where noted. The following solvents were distilled from the listed drying agents: Tetrahydrofuran ( $\mathrm{Na}$, benzophenone), diethyl ether $\left(\mathrm{Na}\right.$, benzophenone), toluene $(\mathrm{Na})$, dichloromethane $\left(\mathrm{CaH}_{2}\right)$. Anhydrous 1,2 dichloroethane was purchased from Aldrich and used under atmosphere of argon. Silver nitrate $(99.9995 \%$ Ag) was purchased from Strem chemicals. Chromatographic purifications were performed on Merck silica gel grade (230-400 mesh) and TLC's were performed on silica coated plastic baked TLC plates from Silicycles. The general solvent systems used were either a combination of ethyl acetate / hexanes or dichloromethane / hexanes unless otherwise specified. Compounds were visualized by dipping in solution of $\mathrm{KMnO}_{4}$ followed by heating with a heat-gun. ${ }^{1} \mathrm{H}$ NMR data obtained either on a Varian $300 \mathrm{MHz}$ or $500 \mathrm{MHz}$ instrument are reported in parts per million $(\delta)$ relative to tetramethyl silane $(0.00 \mathrm{ppm})$ or chloroform (7.24 ppm) for spectra run in $\mathrm{CDCl}_{3}$ and multiplicities are indicated by s (singlet), $\mathrm{d}$ (doublet), t (triplet), q (quartet), dd (doublet of doublets), dt (doublet of triplets) and br (broad). ${ }^{13} \mathrm{C}$ NMR data were obtained on the Varian $300 \mathrm{MHz}$ or $500 \mathrm{MHz}$ instruments respectively and are reported in $\delta$ relative to $\mathrm{CDCl}_{3}(77 \mathrm{ppm})$. Infrared spectra were recorded on Perkin Elmer FT IR instrument and the peaks are reported in $\mathrm{cm}^{-1}$ with indicated relative intensities: $\mathrm{s}$ (strong), $\mathrm{m}$ (medium), w (weak), br (broad). 1-D NOE experiments and NOESY experiments were performed on a Varian $600 \mathrm{MHz}$ NMR instrument. Mass spectral data and elemental analysis were obtained from Michigan State University Biochemistry Mass spectrometry facility and in-house facility. The mass spectra were obtained using direct probe El (Electron impact) using chloroform as solvent on JEOL AX-505 double focusing mass spectometer, FAB (Fast atom bombardment) conditions using 2-Nitrobenzyl alcohol as solvent on JEOL HX-110 double focusing mass spectrometer in the positive ionization mode. Data are reported in the form of $\mathrm{m} / \mathrm{z}$ (intensity relative to base peak $=100$ ). Organ-lithium reagents were purchased from Aldrich and titrated by the Watson-Eastham procedure in benzene as the solvent whereas Grignard reagents purchased from strem chemicals and titrated to a known concentration following the procedure developed by Paquette et.al. ${ }^{1}$ The indicated reaction temperatures are of the oil bath temperature monitored by a digital temperature controller. The reactions were often monitored for completion by quenching an aliquot of the reaction mixture in ether/water and ethereal layer was subjected to GC/MS analyses on Saturn 2000R mass spectrometer and 3800GC using chrome-pack capillary column. Melting points (uncorrected) were recorded on Thomas Hoover capillary melting point apparatus using $1.5-1.8 \times 90 \mathrm{~mm}$ capillary tubes. Substrate $4 \mathrm{c}\left(\mathrm{R}_{4}=\mathrm{OMe}, \mathrm{R}_{2}=\mathrm{Me}\right)$ was prepared in two steps from 2,6 -bis-hydroxy-methyl-p-cresol in $73 \%$ overall yield following a literature procedure. ${ }^{2}$ 
Compound $4 \mathbf{d}\left(\mathrm{R}_{4}=\mathrm{OMe}, \mathrm{R}_{2}=\mathrm{Ph}\right)$ is also a known compound but a slightly different procedure was used for its preparation. ${ }^{3}$

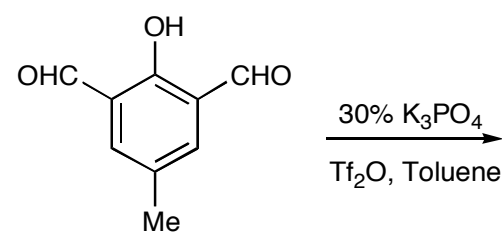<smiles>O=Cc1cc(C=O)c(OC(F)(F)F)c(OC(F)(F)F)c1</smiles>

9

\section{Trifluoromethanesulfonic acid 2,6-diformyl-4-methyl-phenyl ester 9:}

2-Hydroxy-5-methyl-benzene-1,3-dicarbaldehyde was either purchased from Trans-World Chemicals or obtained by manganese dioxide oxidation of 2,6-bis-hydroxymethyl- $p$-cresol just prior to use following the literature procedure. ${ }^{4}$ The following procedure for preparation of the aryl triflate 9 was modified from that reported recently. ${ }^{5}$ The phenol $8(6.1 \mathrm{~g}, 37.2 \mathrm{mmol})$ was dissolved in $30 \%$ aqueous solution of potassium phosphate $(150 \mathrm{~mL})$ and toluene $(75 \mathrm{~mL})$ was added. The resulting solution was red-orange in color and was stirred at ambient temperature for $15 \mathrm{~min}$. Triflic anhydride $(11.2 \mathrm{~mL})$ was added dropwise at $0{ }^{\circ} \mathrm{C}$ with vigorous stirring and the resulting solution was warmed to room temperature and was stirred overnight. The reaction was subjected to an aqueous workup by washing with water $(2 \times 100 \mathrm{~mL})$. The organic layer was dried over anhydrous magnesium sulfate and then concentrated under reduced pressure. Purification by column chromatography yielded $64 \%(7.05 \mathrm{~g})$ of the desired compound as a white flaky solid. The phenol 8 was recovered by eluting with ethyl acetate and re-subjected to the reaction conditions and the overall yield of the triflate 9 could be improved to $83 \%$. $R_{f}=0.15$ (10\% EtOAc / hexanes). $\mathrm{Mp}=57-59{ }^{\circ} \mathrm{C}$. Spectral data for 9: ${ }^{1} \mathrm{H} \mathrm{NMR}\left(\mathrm{CDCl}_{3}, 300 \mathrm{MHz}\right) \delta 2.49(\mathrm{~s}, 3 \mathrm{H}), 8.02(\mathrm{~s}$, 2H), 10.22 (s, 2H); ${ }^{13} \mathrm{C}$ NMR $\left(\mathrm{CDCl}_{3}, 75 \mathrm{MHz}\right) \delta 20.79,118.55$ (q, J = $319 \mathrm{~Hz}$ ), 129.53, 135.91, 140.26, 147.24, 185.55; IR $\left(\mathrm{CH}_{2} \mathrm{Cl}_{2}\right) 3063 w, 2893 w, 1699 s, 1597 s, 1466 \mathrm{~m}, 1435 \mathrm{~s}, 1389 \mathrm{~m}$, $1292 \mathrm{~m}, 1248 \mathrm{~s}, 1203 \mathrm{brs}, 1122 \mathrm{brs} \mathrm{cm} \mathrm{cm}^{-1}$. Anal calcd for $\mathrm{C}_{10} \mathrm{H}_{7} \mathrm{~F}_{3} \mathrm{O}_{5} \mathrm{~S}: \mathrm{C}, 40.55 ; \mathrm{H}, 2.38$. Found: $\mathrm{C}$, 40.64; H, 2.30 .<smiles>Cc1cc(C=O)c(OC(F)(F)F)c(OC(F)(F)F)c1</smiles>

9

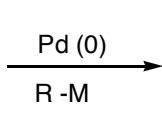<smiles>[R]c1c(C=O)cc(C)cc1C=O</smiles>

10 


\section{Palladium catalyzed Cross Coupling in the Preparation of the Dialdehyde 10.}

4- Methylbiphenyl-2,6-dicarbaldehyde $(R=P h)$ 10a: Aryl triflate $9(6.22 \mathrm{~g}, 21 \mathrm{mmol})$, phenyl boronic acid (282 mg, $23.1 \mathrm{mmol}$ ), tetrakis(triphenylphosphine)palladium (0) (606 mg, 2.5 $\mathrm{mol} \%)$, and anhydrous potassium phosphate $(6.69 \mathrm{~g}, 31.5 \mathrm{~mol})$ were placed in a $250 \mathrm{~mL}$ Schlenk flask under Argon atmosphere. Degassed 1,4-dioxane $(100 \mathrm{~mL})$ was added. The resulting mixture was deoxygenated by the freeze-thaw method (-196 to $25^{\circ} \mathrm{C}, 2$ cycles), backfilled with argon and subsequently stirred at $85^{\circ} \mathrm{C}$ till the conversion was $>95 \%$ as estimated by GC / MS. The mixture was diluted with benzene $(200 \mathrm{~mL})$ and treated with $30 \%$ aqueous basic hydrogen peroxide. The product was extracted with ether $(200 \mathrm{~mL})$ and subsequent removal of solvent under reduced pressure afforded the crude material. Purification by column chromatography on silica gel with $10 \%$ ethyl acetate/hexanes yielded ( $4 \mathrm{~g}, 85 \%$ ) the desired compound $10 \mathrm{a}$ as a white solid. $\mathrm{Mp}=124{ }^{\circ} \mathrm{C} ; \mathrm{R}_{\mathrm{f}}$ (hexanes/ethyl acetate 9/1) $=0.35$. Spectral data for 10a: ${ }^{1} \mathrm{H}$ NMR $\left(\mathrm{CDCl}_{3}, 300 \mathrm{MHz}\right) \delta 2.49(\mathrm{~s}, 3 \mathrm{H}), 7.30-7.49(\mathrm{~m}, 5 \mathrm{H}), 8.03(\mathrm{~s}, 2 \mathrm{H}), 9.77(\mathrm{~s}, 2 \mathrm{H}) ;{ }^{13} \mathrm{C} \mathrm{NMR}\left(\mathrm{CDCl}_{3}\right.$, $75 \mathrm{MHz}) \delta$ 20.84, 128.32, 128.74, 130.87, 132.42, 132.82, 134.56, 138.46, 145.56, 190.95; IR $\left(\mathrm{CH}_{2} \mathrm{Cl}_{2}\right) 3053 w, 3034 w, 2893 w, 2868 w, 2762 w, 1687 \mathrm{~s}, 1560 \mathrm{~m}, 1450 \mathrm{~m}, 1397 \mathrm{~s}, 1230 \mathrm{~s} \mathrm{~cm}^{-1}$; mass spectrum m/z (\% rel intensity) $224 \mathrm{M}^{+}$(100), 195 (93), 181 (43), 165 (60), 152 (55), calcd for $\mathrm{C}_{15} \mathrm{H}_{12} \mathrm{O}_{2} \mathrm{~m} / \mathrm{z}$ 224.0837, measd 224.0836. Anal Calcd for $\mathrm{C}_{15} \mathrm{H}_{12} \mathrm{O}_{2}: \mathrm{C}, 80.34 ; \mathrm{H}, 5.39$. Found: $\mathrm{C}$, 80.08; H, 5.20.

2-Hexyl-5-methyl-1,3-benzenedicarbaldehyde $\left(R=C_{6} H_{13}\right)$ 10b: Aryl triflate $9(4.13 \mathrm{~g}$, $15.18 \mathrm{mmol}$ ) and dichlorobis(triphenylphosphine)palladium II (490 mg, $5 \mathrm{~mol} \%$ ) were transferred to a $100 \mathrm{~mL}$ three necked round bottomed flask and $50 \mathrm{~mL}$ of 1,4-dioxane was added. To another flask was added indium (III) chloride (1.122 g, $5.06 \mathrm{mmol}$ ) which was dried under vacuum with a heat gun. Tetrahydrofuran $45 \mathrm{~mL}$ was then added. Hexyl lithium (2.3M, $15.18 \mathrm{mmol})$ was subsequently added to the flask at $-78{ }^{\circ} \mathrm{C}$. The reaction mixture was then stirred at this temperature for $30 \mathrm{~min}$ and then warmed up to ambient temperature. The resulting trihexyl indium reagent generated in situ was added to the reaction mixture containing aryl triflate 9 and the catalyst in refluxing tetrahydrofuran under argon. The reaction was continued till the disappearance of the starting material was indicated by GC/MS. A few drops of methanol was added to quench the reaction. The mixture was concentrated in vacuo and ether $(100 \mathrm{~mL})$ was added. The organic phase was washed with aqueous hydrochloric acid (10\%, $50 \times 2 \mathrm{~mL}$ ), saturated aqueous sodium bicarbonate $(60 \times 2 \mathrm{~mL})$, and saturated aqueous sodium chloride (60 $\times 2 \mathrm{~mL}$ ), and then dried over anhydrous $\mathrm{MgSO}_{4}$, filtered and concentrated in vacuo. The residue was purified by silica gel chromatography (5\% ethyl acetate / hexanes) to afford, after concentration and drying under high vacuum, $1.65 \mathrm{~g} \mathrm{(47 \% )} \mathrm{of} \mathrm{the} \mathrm{cross-coupled} \mathrm{product} \mathrm{as} \mathrm{an}$ yellow oil. $R_{f}$ (hexanes/ethyl acetate 19/1) $=0.25$. Spectral data for $10 b:{ }^{1} \mathrm{H}$ NMR $\left(\mathrm{CDCl}_{3}\right.$, $300 \mathrm{MHz}) \delta 0.90(\mathrm{t}, 3 \mathrm{H}, \mathrm{J}=6.3 \mathrm{~Hz}), 1.32-1.65(\mathrm{~m}, 8 \mathrm{H}), 2.46(\mathrm{~s}, 3 \mathrm{H}), 3.42(\mathrm{t}, 2 \mathrm{H}, \mathrm{J}=6.9 \mathrm{~Hz})$, 
7.90 (s, $2 \mathrm{H}), 10.39$ (s, $2 \mathrm{H}) ;{ }^{13} \mathrm{C}$ NMR $\left(\mathrm{CDCl}_{3}, 75 \mathrm{MHz}\right) \delta 13.95,20.54,22.49,25.64,29.29$, $31.45,33.49,134.69,136.40,136.56,145.15,191.16$; IR (neat) 2959s, 2928s, 2857s, 2756m, $2731 \mathrm{~m}, 1689 \mathrm{~s}, 1608 \mathrm{~m}, 1570 \mathrm{~s}, 1462 \mathrm{~s}, 1396 \mathrm{~s}, 1281 \mathrm{~m}, 1234 \mathrm{~s}, 1107 \mathrm{~m} \mathrm{~cm}^{-1}$; mass spectrum $\mathrm{m} / \mathrm{z}$ (\% rel. intensity) $232 \mathrm{M}^{+}(20), 175$ (25), 84 (100), calcd for $\mathrm{C}_{15} \mathrm{H}_{20} \mathrm{O}_{2} \mathrm{~m} / \mathrm{z}$ 232.1463, measd 232.1464. Anal Calcd for $\mathrm{C}_{15} \mathrm{H}_{20} \mathrm{O}_{2}: \mathrm{C}, 77.55 ; \mathrm{H}, 8.68$. Found: $\mathrm{C}, 77.10 ; \mathrm{H}, 8.75$.

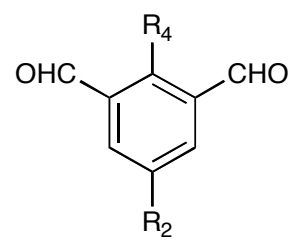

10
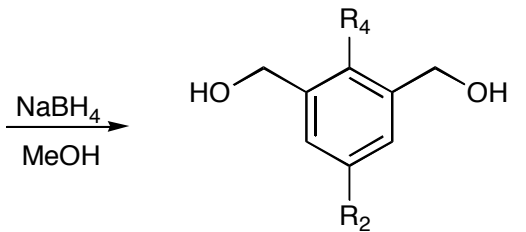

11

\section{Reduction of the Dialdehyde 10 to the bis-Hydroxymethylarene 11.}

(6-Hydroxymethyl-4-methylbiphenyl-2-yl)methanol $\left(R_{4}=P h, R_{2}=M e\right) 11$ a: The aldehyde 10a (3.68 g, $16.42 \mathrm{mmol}$ ) was dissolved in $55 \mathrm{~mL}$ of methanol and 2 eq. of sodium borohydride was added at $0{ }^{\circ} \mathrm{C}$ and subsequently stirred at this temperature for $30 \mathrm{~min}$. The reaction was warmed to room temperature and stirring continued for another hour. The reaction was quenched by addition of water and then the organic layer was extracted into ether. Drying over anhydrous magnesium sulfate followed by removal of the solvent under reduced pressure yielded the product 11a as a white solid in $95 \%$ yield $(3.55 \mathrm{~g})$. Mp $=131-133^{\circ} \mathrm{C}$. Spectral data for 11a: $R_{\mathrm{f}}=0.16$ (hexanes/ethyl acetate 3/1); ${ }^{1} \mathrm{H}$ NMR $\left(\mathrm{CDCl}_{3}, 300 \mathrm{MHz}\right) \delta 2.39(\mathrm{~s}, 3 \mathrm{H}), 3.85(\mathrm{t}$, $2 \mathrm{H}, \mathrm{J}=5.2 \mathrm{~Hz}$ ), 4.26 (d, $4 \mathrm{H}, \mathrm{J}=5.4 \mathrm{~Hz}$ ), 7.17 (d, $2 \mathrm{H}, \mathrm{J}=7.0 \mathrm{~Hz}$ ), $7.348(\mathrm{~s}, 2 \mathrm{H}), 7.352$ (t, $1 \mathrm{H}, \mathrm{J}$ $=7.5 \mathrm{~Hz}), 7.41(\mathrm{t}, 2 \mathrm{H}, \mathrm{J}=7.5 \mathrm{~Hz}) ;{ }^{13} \mathrm{C} \mathrm{NMR}\left(\mathrm{CDCl}_{3}, 75 \mathrm{MHz}\right) \delta 20.84,61.95,126.34,127.11$, 128.33, 129.67, 135.78, 136.65, 138.89, 139.70; IR $\left(\mathrm{CH}_{2} \mathrm{Cl}_{2}\right) 3424 \mathrm{br}, 2926 \mathrm{~m}, 1645 \mathrm{~s}, 1221 \mathrm{w} \mathrm{cm}^{-1}$; mass spectrum $m / z$ (\% rel. intensity) $228 \mathrm{M}^{+}$(100), 210 (97), 192 (93), 181 (97), 165 (97), calcd for $\mathrm{C}_{15} \mathrm{H}_{16} \mathrm{O}_{2} \mathrm{~m} / \mathrm{z}$ 228.1150, measd 228.1154. Anal calcd for $\mathrm{C}_{15} \mathrm{H}_{16} \mathrm{O}_{2}: \mathrm{C}, 78.92 ; \mathrm{H}, 7.06$. Found: C, 78.58; H, 6.99.

(2-Hexyl-3-hydroxymethyl-5-methylphenyl)methanol $\left(R_{4}=C_{6} H_{13}, \quad R_{2}=M e\right) 11$ b: Following the procedure described above for 11a, 11b was isolated in $86 \%$ yield as white powdery solid. $\mathrm{Mp}=78-80{ }^{\circ} \mathrm{C}$. Spectral data for $11 \mathrm{~b}: \mathrm{R}_{\mathrm{f}}=0.19$ (hexanes/ethyl acetate $3 / 1$ ); ${ }^{1} \mathrm{H}$ NMR (CDCl, $300 \mathrm{MHz}) \delta 0.85(\mathrm{t}, 3 \mathrm{H}, \mathrm{J}=6.9 \mathrm{~Hz}), 1.30-1.51(\mathrm{~m}, 10 \mathrm{H}), 2.32(\mathrm{~s}, 3 \mathrm{H}), 2.67(\mathrm{t}, 2 \mathrm{H}$, $\mathrm{J}=6.6 \mathrm{~Hz}), 4.70(\mathrm{~s}, 4 \mathrm{H}), 7.17(\mathrm{~s}, 2 \mathrm{H}) ;{ }^{13} \mathrm{C} \mathrm{NMR}\left(\mathrm{CDCl}_{3}, 75 \mathrm{MHz}\right) \delta 14.08,20.96,22.65,27.97$, $29.9431 .63,31.79,63.18,128.83,135.85,136.09,138.84$; IR $\left(\mathrm{CH}_{2} \mathrm{Cl}_{2}\right) 3350 \mathrm{br}, 3260 \mathrm{br}, 2953 \mathrm{~m}$, 2920s, 2870m, 1469m, 1265s cm $\mathrm{cm}^{-1}$. Anal calcd for $\mathrm{C}_{15} \mathrm{H}_{24} \mathrm{O}_{2}: \mathrm{C}, 76.23 ; \mathrm{H}, 10.24$. Found: $\mathrm{C}$, 75.89; H, 10.43. 
3,5-bis-hydroxymethyl-4-methoxybiphenyl $\left(R_{4}=\right.$ OMe, $\left.R_{2}=P h\right)$ 11d: 2,6-bis(hydroxymethyl)-4-phenylphenol was prepared in $90 \%$ yield by formylation of 4-phenyl phenol under basic conditions. ${ }^{5}$ The phenol was then converted to $11 \mathbf{d}$ following Cram's procedure for the methylation of 2,6-bis-(hydroxymethyl)-p-cresol in $87 \%$ yield. ${ }^{1} \mathrm{R}_{\mathrm{f}}=0.21$ (hexanes/ ethyl acetate $=1 / 1) . \mathrm{Mp}=96-98{ }^{\circ} \mathrm{C}$. Spectral data for 11d: ${ }^{1} \mathrm{H}$ NMR $\left(\mathrm{CDCl}_{3}, 500 \mathrm{MHz}\right) \delta 2.07(\mathrm{~s}, 2 \mathrm{H})$, 3.87 (s, $3 \mathrm{H}$ ), 4.78 (s, 4 H), $7.32(\mathrm{t}, 1 \mathrm{H}, \mathrm{J}=7.5 \mathrm{~Hz}), 7.41$ (t, $2 \mathrm{H}, \mathrm{J}=8.0 \mathrm{~Hz}$ ), 7.546 (s, $2 \mathrm{H}), 7.55$ $(\mathrm{d}, 2 \mathrm{H}, \mathrm{J}=8.0 \mathrm{~Hz}) ;{ }^{13} \mathrm{C}$ NMR $\left(\mathrm{CDCl}_{3}, 75 \mathrm{MHz}\right) 61.10,61.25,126.99,127.27,127.57,128.76$, $134.25,137.79,140.29,155.59$.

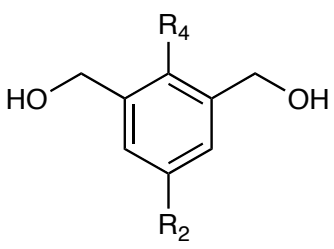

11

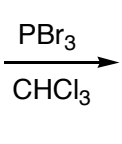<smiles>[R2]c1cc(CBr)c([R1])c(CBr)c1</smiles>

4

\section{Conversion of the bis-Hydroxymethylarenes 11 to the bis-Bromomethylarenes 4.}

2,6-bis-bromomethyl-4-methylbiphenyl $\left(R_{4}=P h, R_{2}=M e\right) 4 a: \quad T h e$ alcohol $(3.55 \mathrm{~g}$, $15.57 \mathrm{mmol}$ ) 11a was dissolved in $65 \mathrm{~mL}$ of chloroform and phosphorus tribromide (3.6 mL, 2.4 eq) was added dropwise and the mixture was stirred at ambient temperature until complete conversion to the product had occurred. The reaction was quenched by the addition of saturated aqueous sodium bicarbonate $(50 \mathrm{~mL})$ and the organic layer washed once with water $(100 \mathrm{~mL})$. Concentration of the solvent under vacuum gave the desired product as yellow oil. Purification by silica gel chromatography (10\% dichloromethane/hexanes) gave the bromide $\mathbf{4 a}$ as a white solid in $86 \%$ yield $\left(4.74 \mathrm{~g}\right.$ ). $\mathrm{Mp}=101-103{ }^{\circ} \mathrm{C} . \mathrm{R}_{\mathrm{f}}=0.34$ (hexanes/dichloromethane $=9 / 1$ ) Spectral data for 4a: ${ }^{1} \mathrm{H} \mathrm{NMR}\left(\mathrm{CDCl}_{3}, 300 \mathrm{MHz}\right) \delta 2.38(\mathrm{~s}, 3 \mathrm{H}), 4.16(\mathrm{~s}, 4 \mathrm{H}), 7.27(\mathrm{~s}, 2 \mathrm{H})$, 7.32-7.42 (m, $\left.5 \mathrm{H}\right)$; ${ }^{13} \mathrm{C}$ NMR $\left(\mathrm{CDCl}_{3}, 75 \mathrm{MHz}\right) \delta 20.96,31.96,127.87,128.27,129.49,131.32$, 136.54,136.56,138.27,139.02; IR $\left(\mathrm{CH}_{2} \mathrm{Cl}_{2}\right) 3053 \mathrm{~m}, 2982 \mathrm{~m}, 1614 \mathrm{w}, 1458 \mathrm{~m}, 1442 \mathrm{~m}, 1285 \mathrm{~s}, 1213 \mathrm{~s}$ $\mathrm{cm}^{-1}$; mass spectrum $\mathrm{m} / \mathrm{z}$ (\% rel intensity) $351.94 \mathrm{M}^{+}\left({ }^{79} \mathrm{Br},{ }^{79} \mathrm{Br}, 6.6\right), 353.94 \mathrm{M}^{+}\left({ }^{79} \mathrm{Br},{ }^{81} \mathrm{Br}, 13\right.$ ), $355.94 \mathrm{M}^{+}\left({ }^{81} \mathrm{Br},{ }^{81} \mathrm{Br}, 6.5\right), 193$ (100), 178 (35), 83 (60), calcd for $\mathrm{C}_{15} \mathrm{H}_{14} \mathrm{Br}_{2} \mathrm{~m} / \mathrm{z}$ 351.9462, measd 351.9441. Anal calcd for $\mathrm{C}_{15} \mathrm{H}_{14} \mathrm{Br}_{2}: \mathrm{C}, 50.88 ; \mathrm{H}, 3.99$. Found: $\mathrm{C}, 50.98 ; \mathrm{H}, 3.90$.

1,3- Bis-bromomethyl-2-hexyl-5-methylbenzene $\left(R_{4}=C_{6} H_{13}, R_{2}=M e\right) 4 \boldsymbol{b}:$ The procedure described above for $\mathbf{4 a}$ was followed and $\mathbf{4 b}$ was isolated as a white solid in $59 \%$ yield. $\mathrm{Mp}=47-49{ }^{\circ} \mathrm{C} . \mathrm{R}_{\mathrm{f}}=0.44$ (hexanes/dichloromethane $=9 / 1$ ). Spectral data for $4 \mathrm{~b}:{ }^{1} \mathrm{H}$ NMR $\left(\mathrm{CDCl}_{3}, 300 \mathrm{MHz}\right) \delta 0.92(\mathrm{t}, 3 \mathrm{H}, \mathrm{J}=7.1 \mathrm{~Hz}), 1.33(\mathrm{~m}, 4 \mathrm{H}), 1.52(\mathrm{~m}, 2 \mathrm{H}), 1.64(\mathrm{~m}, 2 \mathrm{H}), 2.28(\mathrm{~s}, 3$ $\mathrm{H}), 2.80(\mathrm{t}, 2 \mathrm{H}, \mathrm{J}=8.1 \mathrm{~Hz}), 4.5(\mathrm{~s}, 4 \mathrm{H}), 7.13(\mathrm{~s}, 2 \mathrm{H}) ;{ }^{13} \mathrm{C} \mathrm{NMR}\left(\mathrm{CDCl}_{3}, 75 \mathrm{MHz}\right) \delta$ 14.07, 20.62, 22.61, 28.45, 29.89, 31.33, 31.55, 31.69, 132.31, 136.31, 136.39, 137.91; IR $\left(\mathrm{CH}_{2} \mathrm{Cl}_{2}\right) 3049 \mathrm{w}$, 2955s, 2926s, 2860s, 1479m, 1462m, 1265s, $1209 \mathrm{~s} \mathrm{~cm}^{-1}$; mass spectrum $\mathrm{m} / \mathrm{z}$ (\% rel. intensity) 
$360.01 \mathrm{M}^{+}\left({ }^{79} \mathrm{Br},{ }^{79} \mathrm{Br}, 51\right), 362.01 \mathrm{M}^{+}\left({ }^{79} \mathrm{Br},{ }^{81} \mathrm{Br}, 100\right), 364.01 \mathrm{M}^{+}\left({ }^{81} \mathrm{Br},{ }^{81} \mathrm{Br}, 50\right), 281$ (15), 211 (100), 132 (43), 91 (13), calcd for $\mathrm{C}_{15} \mathrm{H}_{22} \mathrm{Br}_{2} \mathrm{~m} / \mathrm{z} 360.0088$, measd 360.0089. Anal calcd for $\mathrm{C}_{15} \mathrm{H}_{22} \mathrm{Br}_{2}: \mathrm{C}, 49.75 ; \mathrm{H}, 6.12$. Found: $\mathrm{C}, 49.86 ; \mathrm{H}, 6.44$.

3,5-Bis-bromomethyl-4-methoxybiphenyl $\left(R_{4}=\mathrm{OMe}, R_{2}=P h\right) 4 d$ : The procedure above for $\mathbf{4 a}$ was followed and $\mathbf{4 d}$ was isolated as white solid in $79 \%$ yield. $\mathrm{Mp}=100-103^{\circ} \mathrm{C}$. Spectral data for 4d: ${ }^{1} \mathrm{H}$ NMR $\left(\mathrm{CDCl}_{3}, 300 \mathrm{MHz}\right) \delta 3.93(\mathrm{~s}, 3 \mathrm{H}), 4.48(\mathrm{~s}, 4 \mathrm{H}), 7.23(\mathrm{t}, 1 \mathrm{H}, \mathrm{J}=6.9 \mathrm{~Hz}), 7.31$ (t, $2 \mathrm{H}, \mathrm{J}=7.2 \mathrm{~Hz}), 7.42(\mathrm{~d}, 2 \mathrm{H}, \mathrm{J}=7.2 \mathrm{~Hz}), 7.46(\mathrm{~s}, 2 \mathrm{H}) ;{ }^{13} \mathrm{C} \mathrm{NMR}\left(\mathrm{CDCl}_{3}, 75 \mathrm{MHz}\right) \delta 27.56$, 62.36, 126.94, 127.62, 128.86, 130.84, 132.19, 138.19, 139.45, 155.91; mass spectrum $\mathrm{m} / \mathrm{z}$ (\% rel intensity) $372 \mathrm{M}^{+}\left({ }^{81} \mathrm{Br},{ }^{81} \mathrm{Br}, 50\right), 370 \mathrm{M}^{+}\left({ }^{79} \mathrm{Br},{ }^{81} \mathrm{Br}, 100\right), 368 \mathrm{M}^{+}\left({ }^{79} \mathrm{Br},{ }^{79} \mathrm{Br}, 51\right), 291$ (93), 289 (094), 182 (45), 181 (100).

\section{Preparation of the Silyl Substituted Diynes 5.}

Method A<smiles>[R]c1cc(CBr)c([R2])c(CBr)c1</smiles>

4

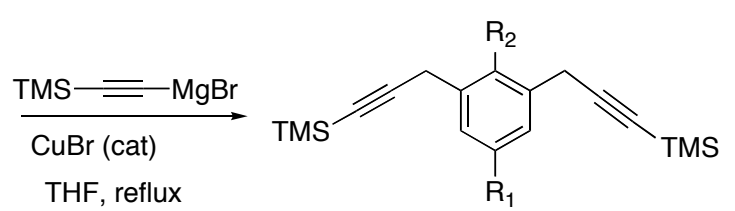

5

Trimethylsilylethynylmagnesium bromide ${ }^{6}(8 \mathrm{eq})$ was generated in situ by the addition of ethylmagnesium bromide ( $3 \mathrm{M}$ solution in THF, $8 \mathrm{eq}$ ) to ethynyltrimethylsilane $(8 \mathrm{eq})$ in tetrahydrofuran $(c=1.6-2.4 \mathrm{M})$ at $0{ }^{\circ} \mathrm{C}$ followed by stirring at this temperature for $30 \mathrm{~min}$. The resulting slurry was stirred at room temperature over $0.5 \mathrm{~h}$. Copper (I) bromide (15 mol \%) was added and stirred for another $0.5 \mathrm{~h}$. Benzyl halides $4(1 \mathrm{mmol})$ were subsequently added and then the reaction mixture was refluxed until disappearance of the starting material was indicated by thin-layer chromatography or GC / MS. Saturated ammonium chloride (40 mL) was then added to quench the reaction and the organic layer was separated and extracted with ether $(2 \mathrm{x}$ $50 \mathrm{~mL}$ ). The combined organic layer was washed once with water (equal volume) and then dried over anhydrous magnesium sulfate. The resultant organic layer was filtered through a silica gel pad and stripped of solvent under reduced pressure to give the crude compound $\mathbf{5}$. For consistency in yields, it is highly important that the starting benzyl halides are purified and the reagents are either freshly prepared or titrated to verify concentration. 


\section{Method B}

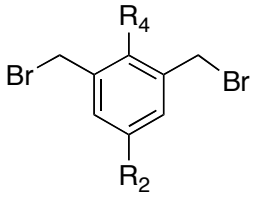

4

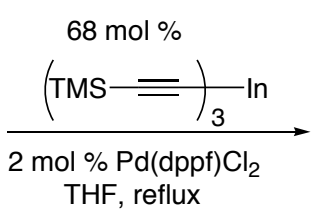

THF, reflux

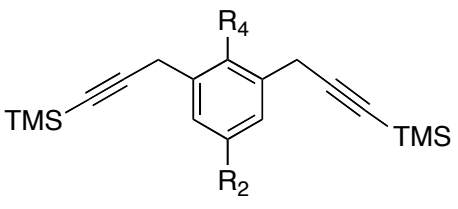

5

Trimethylsilylethynyllithium ${ }^{6}(19.2 \mathrm{mmol})$ was prepared by addition of $n$-butyllithium (12 $\mathrm{mL}, 19.2 \mathrm{mmol})$ to trimethylsilylacetylene $(3 \mathrm{~mL}, 21.22 \mathrm{mmol})$ in tetrahydrofuran $(20 \mathrm{~mL})$ in a 100 $\mathrm{mL}$ flame-dried round bottom flask under argon at $-78{ }^{\circ} \mathrm{C}$. The resulting solution was allowed to warm to room temperature for 10 to $15 \mathrm{~min}$. Indium (III) chloride (1.42 g, $6.4 \mathrm{mmol}$ ) was added to a three-necked $100 \mathrm{~mL}$ round bottom flask and dried under vacuum with a heat gun. Positive argon pressure was then established and THF $(30 \mathrm{~mL})$ was added. The resulting solution was cooled to $-78{ }^{\circ} \mathrm{C}$ and trimethylsilylethynyllithium was added drop-wise via syringe. The mixture was subsequently warmed to room temperature. The bis-benzyl halide 4 (8 $\mathrm{mmol})$ and $\mathrm{Pd}(\mathrm{dppf}) \mathrm{Cl}_{2}$ (131 mg, $0.16 \mathrm{mmol}$ ) were introduced into a flame-dried three-necked $200 \mathrm{~mL}$ roundbottomed flask and THF (32 $\mathrm{mL}$ ) was added. The solution of trialkynylindium reagent was added to this flask under refluxing conditions and the reaction was continued until the disappearance of the starting material was determined as monitored by $\mathrm{GC} /$ mass spec. The reaction was then quenched by the addition of $50 \mathrm{~mL}$ of methanol and the solvent was removed under vacuum. Ether $(200 \mathrm{~mL})$ was added and the organic layer was washed with $10 \%$ hydrochloric acid $(2 \mathrm{x}$ $100 \mathrm{~mL})$, saturated sodium bicarbonate $(2 \times 100 \mathrm{~mL})$ and saturated sodium chloride solution $(2 \mathrm{x}$ $100 \mathrm{~mL}$ ). The resulting solution was filtered through a pad of silica gel to remove any inorganic impurities and upon concentration under reduced pressure the crude product was obtained. Indium trichloride is extremely moisture sensitive and therefore needs to be handled carefully in order to obtain the best results.

4-Methyl-2,6-bis-[(3-(trimethylsilanyl)prop-2-ynyl]biphenyl $\left(R_{4}=P h, \quad R_{2}=M e\right) 5$ a : Following method $\mathrm{A}$, this compound was obtained in $71 \%$ yield as a yellow oil after purification by silica gel chromatography ( $15 \%$ dichloromethane/hexanes). Following method $\mathrm{B}$, an $81 \%$ yield of $5 \mathrm{a}$ was obtained. The resulting oil if left in the freezer at $-20^{\circ} \mathrm{C}$ became a light yellow solid. $\mathrm{Mp}$ $=55-58^{\circ} \mathrm{C} \cdot \mathrm{R}_{\mathrm{f}}$ (hexanes/dichloromethane 85/15) $=0.45$. Spectral data for 5a: ${ }^{1} \mathrm{H}$ NMR $\left(\mathrm{CDCl}_{3}\right.$, $300 \mathrm{MHz}) \delta 0.14(\mathrm{~s}, 18 \mathrm{H}), 2.41(\mathrm{~s}, 3 \mathrm{H}), 3.23(\mathrm{~s}, 4 \mathrm{H}), 7.12(\mathrm{~d}, 2 \mathrm{H}, \mathrm{J}=7.3 \mathrm{~Hz})$, 7.3-7.4 (m, $5 \mathrm{H})$; ${ }^{13} \mathrm{C} \mathrm{NMR}\left(\mathrm{CDCl}_{3}, 75 \mathrm{MHz}\right) \delta 0.14,21.32,24.90,86.69,104.94,127.22,127.59,128.54,129.39$, 134.42, 137.44, 137.59, 138.57; IR (neat) 2959s, 2899m, 2176s, $1466 \mathrm{~m}, 1410 \mathrm{~m}, 1250 \mathrm{~s} \mathrm{~cm}^{-1}$; mass spectrum $\mathrm{m} / \mathrm{z}$ (\% rel intensity) $388 \mathrm{M}^{+}$(8), 315 (18), 285 (15), 179 (17), 83 (100), calcd for $\mathrm{C}_{15} \mathrm{H}_{22} \mathrm{Si}_{2} \mathrm{~m} / \mathrm{z}$ 388.2043, measd 388.2044. 
2- Hexyl-5-methyl-1,3-bis-[3-(trimethylsilanyl)prop-2-ynyl]benzene $\left(R_{4}=C_{6} H_{13}, R_{2}=C_{3}\right)$ $5 \boldsymbol{b}$ : $\quad$ Following method $A$ and purification by silica gel chromatography with $15 \%$ dichloromethane/hexanes afforded the desired compound as a yellow oil in $73 \%$ yield. $R_{f}$ (dichloromethane/hexanes $=15 / 85)=0.49$. Spectral data for $5 \mathbf{b}:{ }^{1} \mathrm{H} \mathrm{NMR}\left(\mathrm{CDCl}_{3}, 300 \mathrm{MHz}\right) \delta$ $0.19(\mathrm{~s}, 18 \mathrm{H}), 0.95(\mathrm{t}, 3 \mathrm{H}, \mathrm{J}=6.5 \mathrm{~Hz}), 1.36-1.53(\mathrm{~m}, 8 \mathrm{H}), 2.35(\mathrm{~s}, 3 \mathrm{H}), 2.64(\mathrm{t}, 2 \mathrm{H}, \mathrm{J}=8.0 \mathrm{~Hz})$, 3.62 (s, $4 \mathrm{H}), 7.23$ (s, $2 \mathrm{H}) ;{ }^{13} \mathrm{C} \mathrm{NMR}\left(\mathrm{CDCl}_{3}, 75 \mathrm{MHz}\right) \delta$ 0.04, 14.10, 20.96, 22.72, 24.04, 28.55, 29.73, 29.97, 31.61, 86.79, 104.85, 128.56, 134.37, 135.45, 135.52; IR (neat) 2989s, 2176s, $1614 \mathrm{~m}, 1468 \mathrm{~s}, 1410 \mathrm{~s}, 1250 \mathrm{~s} \mathrm{~cm}{ }^{-1}$; mass spectrum, calcd for $\mathrm{C}_{25} \mathrm{H}_{40} \mathrm{Si}_{2} \mathrm{~m} / \mathrm{z} 396.2669$, measd 396.2664. Anal calcd for $\mathrm{C}_{25} \mathrm{H}_{40} \mathrm{Si}_{2}: \mathrm{C}, 75.68 ; \mathrm{H}, 10.16$. Found: $\mathrm{C}, 75.76 ; \mathrm{H}, 9.87$.

2-Methoxy-5-methyl-1,3-bis-[3-(trimethylsilanyl)prop-2-ynyl]benzene $\left(R_{4}=\mathrm{OCH}_{3}, \quad R_{2}=\right.$ $\mathrm{CH}_{3}$ ) 5c: Following method $\mathrm{B}$, the residue was purified by silica gel chromatography (20\% dichloromethane/hexanes) to afford the product as colorless oil in $80 \%$ yield. $R_{f}=0.36$ (hexanes/dichloromethane $=4 / 1)$. Spectral data for 5c: ${ }^{1} \mathrm{H}$ NMR $\left(\mathrm{CDCl}_{3}, 300 \mathrm{MHz}\right) \delta 0.22(\mathrm{~s}, 18$ $\mathrm{H}), 2.37$ (s, $3 \mathrm{H}), 3.65$ (s, $4 \mathrm{H}$ ), 3.79 (s, $3 \mathrm{H}), 7.23(\mathrm{~s}, 2 \mathrm{H}) ;{ }^{13} \mathrm{C} \mathrm{NMR}\left(\mathrm{CDCl}_{3}, 75 \mathrm{MHz}\right) \delta 0.04$, 20.47, 20.91, 61.02, 86.49, 104.64, 128.98, 129.16, 133.82, 153.05; IR (neat) 2961s, 2901m, $2832 \mathrm{w}, 2178 \mathrm{~s}, 1482 \mathrm{~s}, 1250 \mathrm{~s} \mathrm{~cm}^{-1}$; mass spectrum $\mathrm{m} / \mathrm{z}$ (\% rel intensity) $342 \mathrm{M}^{+}$(100), 327 (80), 239 (50), 156 (35), 73 (90), calcd for $\mathrm{C}_{20} \mathrm{H}_{30} \mathrm{OSi}_{2} \mathrm{~m} / \mathrm{z}$ 342.1835, measd 342.1835.

4-Methoxy-3,5-bis-[3-(trimethylsilanyl)prop-2-ynyl]biphenyl $\left(R_{4}=\mathrm{OCH}_{3}, R_{2}=P h\right) \mathbf{5}$ : Following method $B$, the crude product was purified by silica gel chromatography $(20 \%$ dichloromethane/hexanes) to give a $77 \%$ yield of $\mathbf{5 d}$ as a white solid. $\mathrm{Mp}=72-74{ }^{\circ} \mathrm{C} . \mathrm{R}_{\mathrm{f}}=0.31$ (hexanes/dichloromethane 4/1). Spectral data for 5d: ${ }^{1} \mathrm{H}$ NMR $\left(\mathrm{CDCl}_{3}, 300 \mathrm{MHz}\right) \delta 0.08(\mathrm{~s}, 18 \mathrm{H})$, 3.59 (s, 4 H), 3.69 (s, 3 H), 7.21 (t, $1 \mathrm{H}, \mathrm{J}=7.2 \mathrm{~Hz}$ ), 7.32 (t, $2 \mathrm{H}, \mathrm{J}=7.5 \mathrm{~Hz}$ ), 7.49 (d, $2 \mathrm{H}, \mathrm{J}=7.2$ $\mathrm{Hz}), 7.57$ (s, $2 \mathrm{H}) ;{ }^{13} \mathrm{C} \mathrm{NMR}\left(\mathrm{CDCl}_{3}, 75 \mathrm{MHz}\right) \delta 0.05,20.75,60.89,87.12,104.34,126.85,127.08$, 127.13, 128.73, 129.87, 137.19, 140.64, 154.86; IR (neat) 3130m, 3100m, 2957s, 2899s, 2178s, $1690 \mathrm{w}, 1590 \mathrm{w}, 1456 \mathrm{~s}, 1404 \mathrm{~s}, 1248 \mathrm{~s} \mathrm{~cm}^{-1}$. Anal calcd for $\mathrm{C}_{25} \mathrm{H}_{32} \mathrm{OSi}_{2}: \mathrm{C}, 74.20 ; \mathrm{H}, 7.97$. Found: C, $74.51 ; \mathrm{H}, 7.89$.

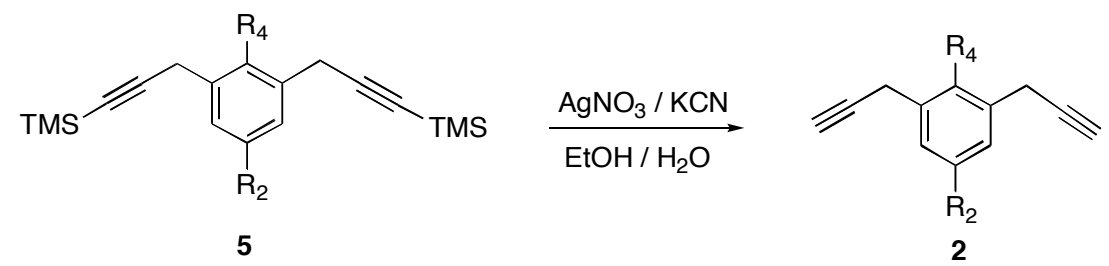

\section{Preparation of the Diynes 2 by Desilylation of 5.}

A solution of the diyne $5(1 \mathrm{mmol})$ in ethanol $(4.5 \mathrm{~mL})$ was added to a $12 \mathrm{~mL}$ aqueous ethanol solution (ethanol $/$ water $=2.3 / 1 \mathrm{v} / \mathrm{v}$ ) of silver nitrate $(3 \mathrm{eq})$ whereupon a milky white 
precipitate appeared immediately upon addition indicating the presence of silver acetylide. The resulting slurry was stirred shielded from light at ambient temperature for $5 \mathrm{~h}$ or overnight. A solution of potassium cyanide $(8 \mathrm{eq})$ in $1 \mathrm{~mL}$ of water was then added. The mixture was stirred for another hour and then the solution was diluted with $100 \mathrm{~mL}$ ether. The organic layer was washed with water $(4 \times 25 \mathrm{~mL})$, brine $(4 \times 25 \mathrm{~mL})$, dried over anhydrous magnesium sulfate and concentrated under vacuum. Purification was either achieved by column chromatography on silica gel or by crystallization.

4-Methyl-2,6-diprop-2-ynylbiphenyl $\left(R_{4}=P h, R_{2}=M e\right) 2 a$ : The product was purified by silica gel chromatography (10\% dichloromethane in hexanes) and obtained as a white solid in 77 $\%$ yield. [Note: This compound is light sensitive; the white solid turns orange over a few weeks]. $\mathrm{Mp}=36-39{ }^{\circ} \mathrm{C} . \mathrm{R}_{\mathrm{f}}=0.28$ (dichloromethane/hexanes = 1/9). Spectral data for $2 \mathrm{a}:{ }^{1} \mathrm{H}$ NMR $\left(\mathrm{CDCl}_{3}, 300 \mathrm{MHz}\right) \delta 2.16(\mathrm{~s}, 2 \mathrm{H}), 2.48(\mathrm{~s}, 3 \mathrm{H}), 3.26(\mathrm{~s}, 4 \mathrm{H}), 7.19(\mathrm{~d}, 2 \mathrm{H}, \mathrm{J}=7.8 \mathrm{~Hz}), 7.43-7.48$ $(\mathrm{m}, 5 \mathrm{H}) ;{ }^{13} \mathrm{C} \mathrm{NMR}\left(\mathrm{CDCl}_{3}, 75 \mathrm{MHz}\right) \delta 21.26,23.34,70.39,82.45,127.39,127.59,128.71$, 129.37, 134.33, 137.45, 137.77, 138.60; IR $\left(\mathrm{CH}_{2} \mathrm{Cl}_{2}\right) 3300 \mathrm{~m}, 2918 \mathrm{~s}, 2849 \mathrm{~s}, 2120 \mathrm{w}, 1736 \mathrm{~m}$, $1464 \mathrm{~m}, 1246 \mathrm{~m} \mathrm{~cm}^{-1}$; mass spectrum $\mathrm{m} / \mathrm{z}$ (\% rel intensity) $244 \mathrm{M}^{+}$(80), 229 (100), 205 (55), 189 (40), 101 (30), calcd for $\mathrm{C}_{19} \mathrm{H}_{16} \mathrm{~m} / \mathrm{z}$ 244.1252, measd 244.1256. Anal calcd for $\mathrm{C}_{19} \mathrm{H}_{16}: \mathrm{C}, 93.4 ; \mathrm{H}$, 6.60. Found: $\mathrm{C}, 92.95 ; \mathrm{H}, 6.54$.

2-Hexyl-5-methyl-1,3-diprop-2-ynylbenzene $\left(R_{4}=C_{6} H_{13}, R_{2}=M e\right) 2 \boldsymbol{b}$ : The crude product was purified by silica gel chromatography (hexanes) in $73 \%$ yield as colorless oil. $R_{f}$ (hexanes) $=0.32$. Spectral data for $\mathbf{2 b}$ : ${ }^{1} \mathrm{H}$ NMR $\left(\mathrm{CDCl}_{3}, 300 \mathrm{MHz}\right) \delta 0.95(\mathrm{t}, 3 \mathrm{H}, \mathrm{J}=6.6 \mathrm{~Hz})$, 1.37-1.49 (m, 8 H), 2.21-2.24 (m, 2 H), 2.37 (s, $3 \mathrm{H}), 2.67$ (t, $2 \mathrm{H}, \mathrm{J}=6.6 \mathrm{~Hz}), 3.59$ (s, $4 \mathrm{H}), 7.26$ (s, $2 \mathrm{H}) ;{ }^{13} \mathrm{C} \mathrm{NMR}\left(\mathrm{CDCl}_{3}, 75 \mathrm{MHz}\right) \delta 14.06,20.92,22.54,22.62,28.54,29.89,29.96,31.58$, 70.53, 82.36, 128.56, 134.24, 135.29, 135.82; IR (neat) 3304s, 2926s, 2856s, 2120w, 1684m, $1466 \mathrm{~m} \mathrm{~cm}^{-1}$; mass spectrum $\mathrm{m} / \mathrm{z}$ (\% rel intensity) $252 \mathrm{M}^{+}(70), 181$ (100), 165 (100), 141 (75), 128 (65), 115 (50), calcd for $\mathrm{C}_{19} \mathrm{H}_{24} \mathrm{~m} / \mathrm{z} 252.1878$, measd 252.1880. Anal calcd for $\mathrm{C}_{19} \mathrm{H}_{24}: \mathrm{C}$, 90.42; H, 9.58. Found: C, 90.03; H, 9.82 .

2-Methoxy-5-methyl-1,3-diprop-2-ynylbenzene $\left(R_{4}=O M e, R_{2}=M e\right) 2 c:$ The crude product was purified by crystallization from hexanes at $0{ }^{\circ} \mathrm{C}$ and obtained as a white solid in $91 \%$ yield. $\mathrm{Mp}=40-42{ }^{\circ} \mathrm{C} . \mathrm{R}_{\mathrm{f}}=0.25\left(20 \% \mathrm{CH}_{2} \mathrm{Cl}_{2} /\right.$ hexanes $)$. Spectral data for $2 \mathrm{c}:{ }^{1} \mathrm{H} \mathrm{NMR}\left(\mathrm{CDCl}_{3}\right.$,

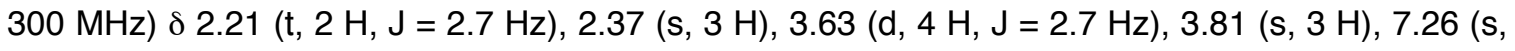
$2 \mathrm{H}) ;{ }^{13} \mathrm{C} \mathrm{NMR}\left(\mathrm{CDCl}_{3}, 75 \mathrm{MHz}\right) \delta 19.05,20.79,61.03,70.06,82.16,129.06,134.17,152.99$ (1 aryl carbon not observed); IR $\left(\mathrm{CH}_{2} \mathrm{Cl}_{2}\right) 3294 \mathrm{~s}, 2943 \mathrm{~m}, 2830 \mathrm{w}, 2150 \mathrm{w}, 1653 \mathrm{w}, 1479 \mathrm{~s}, 1223 \mathrm{~m} \mathrm{~cm}^{-}$ 1. Anal calcd for $\mathrm{C}_{14} \mathrm{H}_{14} \mathrm{O}: \mathrm{C}, 84.81 ; \mathrm{H}, 7.12$. Found: $\mathrm{C}, 84.84 ; \mathrm{H}, 6.94$.

4-Methoxy-3,5-prop-2-ynylbiphenyl $\left(R_{4}=O M e, R_{2}=P h\right) 2 d$ : The crude product was purified by crystallization from hexanes at $0{ }^{\circ} \mathrm{C}$ which gave $\mathbf{2 d}$ as a white solid in $90 \%$ yield. Mp 
$=64-67{ }^{\circ} \mathrm{C} . \mathrm{R}_{\mathrm{f}}=0.21\left(20 \% \mathrm{CH}_{2} \mathrm{Cl}_{2} /\right.$ hexanes $)$. Spectral data for $2 \mathrm{~d}:{ }^{1} \mathrm{H} \mathrm{NMR}\left(\mathrm{CDCl}_{3}, 300 \mathrm{MHz}\right)$

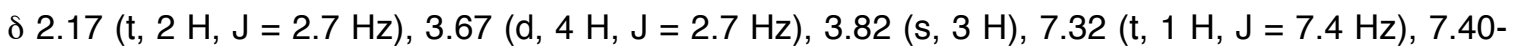
$7.45(\mathrm{t}, 2 \mathrm{H}, \mathrm{J}=7.4 \mathrm{~Hz}), 7.58(\mathrm{~d}, 2 \mathrm{H}, \mathrm{J}=7.4 \mathrm{~Hz}), 7.64(\mathrm{~s}, 2 \mathrm{H}) ;{ }^{13} \mathrm{C} \mathrm{NMR}\left(\mathrm{CDCl}_{3}, 75 \mathrm{MHz}\right) \delta$ $19.35,61.12,70.51,81.88,127.09,127.32$, 128.72, 129.77, 137.66, 140.54, 154.76, 156.99; IR $\left(\mathrm{CH}_{2} \mathrm{Cl}_{2}\right) 3294 \mathrm{~s}, 2941 \mathrm{w}, 2831 \mathrm{w}, 2120 \mathrm{w}, 1686 \mathrm{w}, 1473 \mathrm{~s}, 1240 \mathrm{~m} \mathrm{~cm}{ }^{-1}$. Anal calcd for $\mathrm{C}_{19} \mathrm{H}_{16} \mathrm{O}: \mathrm{C}$, 87.66; $\mathrm{H}, 6.19$. Found: $\mathrm{C}, 87.27 ; \mathrm{H}, 6.36$.

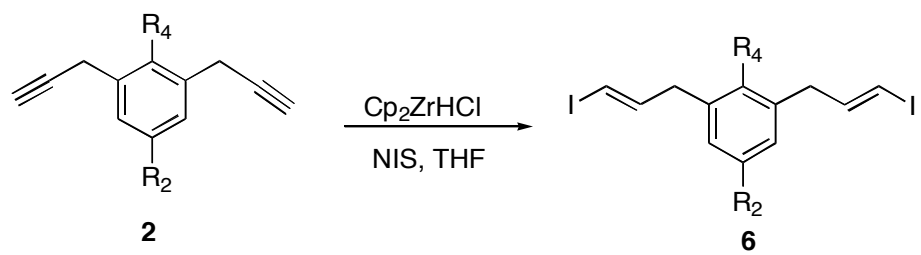

\section{Synthesis of the bis-(E)-Vinyl lodides 6 by Hydrozirconation.}

Schwartz's reagent was prepared following a literature procedure: ${ }^{7}$ a solution of biscyclopentadienylzirconium dichloride $(4 \mathrm{eq})$ in tetrahydrofuran $(c=0.12-0.15 \mathrm{M})$ was treated with Super Hydride (4 eq) and subsequently stirred for $1 \mathrm{~h}$ shielded from light. To this freshly prepared Schwartz reagent was added the diyne $(1 \mathrm{mmol})$ and the mixture was stirred at room temperature for $1 \mathrm{~h}$. N-lodosuccinimide (4 eq) was subsequently added and stirring continued for $4 \mathrm{~h}$. The reaction was quenched by pouring it into saturated sodium bicarbonate solution (40 $\mathrm{mL})$. A solution of $10 \%$ ethyl acetate/hexanes $(100 \mathrm{~mL})$ was added and the organic layer separated and washed with brine $(40 \mathrm{~mL})$, dried over anhydrous magnesium sulfate. After filtration through a bed of Celite atop a short plug of silica gel with $10 \%$ ethyl acetate/hexanes the solvents were removed to give the crude compound as a oil. Purification by column chromatography on silica gel afforded the desired product as a clear oily liquid.

2,6-Bis-(3-iodoallyl)-4-methylbiphenyl $\left(R_{4}=P h, R_{2}=M e\right) 6 a$ : The product was purified by silica gel chromatography (15\% dichloromethane/hexanes) and obtained in $73 \%$ yield as a colorless oil. $\mathrm{R}_{\mathrm{f}}$ (hexanes/dichloromethane 85/15) $=0.4$. Spectral data for $6 \mathrm{a}:{ }^{1} \mathrm{H}$ NMR $\left(\mathrm{CDCl}_{3}\right.$,

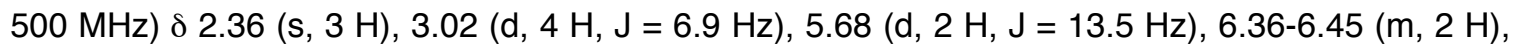
$6.95(\mathrm{~s}, 2 \mathrm{H}), 7.05$ (d, $2 \mathrm{H}, \mathrm{J}=6.5 \mathrm{~Hz}), 7.33(\mathrm{t}, 1 \mathrm{H}, \mathrm{J}=7.0 \mathrm{~Hz}), 7.38(\mathrm{t}, 2 \mathrm{H}, \mathrm{J}=6.5 \mathrm{~Hz}) ;{ }^{13} \mathrm{C} \mathrm{NMR}$ $\left(\mathrm{CDCl}_{3}, 75 \mathrm{MHz}\right) \delta 21.12,40.26,75.96,127.22,128.26,128.36,129.61,136.46,137.52,138.64$, 139.06, 144.59; IR (neat) 3053m, 3020m, 2918s, 2853m, 1611s, 1466s, 1441s, $1209 \mathrm{~s} \mathrm{~cm}^{-1}$; mass spectrum $m / z$ (\% rel intensity) $500 \mathrm{M}^{+}$(30), 374 (100), 247 (65), 206 (80), 85 (55), calcd for $\mathrm{C}_{19} \mathrm{H}_{18} \mathrm{l}_{2} \mathrm{~m} / \mathrm{z}$ 499.9498, measd 499.9499.

2-Hexyl-1,3-bis-(3-iodoallyl)-5-methylbenzene $\left(R_{4}=C_{6} H_{13}, R_{2}=M e\right) 6 b$ : The product was purified by silica gel chromatography (hexanes) and obtained in $77 \%$ yield as a colorless oil. $R_{f}$ (hexanes) $=0.24$. Spectral data for $6 \mathbf{b}:{ }^{1} \mathrm{H}$ NMR $\left(\mathrm{CDCl}_{3}, 300 \mathrm{MHz}\right) \delta 0.92-0.96(\mathrm{t}, 3 \mathrm{H}, \mathrm{J}=6.4$ 
$\mathrm{Hz}), 1.34-1.42(\mathrm{~m}, 8 \mathrm{H}), 2.31(\mathrm{~s}, 3 \mathrm{H}), 2.53(\mathrm{t}, 2 \mathrm{H}, \mathrm{J}=7.3 \mathrm{~Hz}), 3.38(\mathrm{~d}, 4 \mathrm{H}, \mathrm{J}=6.6 \mathrm{~Hz}), 6.02(\mathrm{~d}, 2$ $\mathrm{H}, \mathrm{J}=14.5 \mathrm{~Hz}), 6.63-6.78(\mathrm{~m}, 2 \mathrm{H}), 6.87(\mathrm{~s}, 2 \mathrm{H}) ;{ }^{13} \mathrm{C} \mathrm{NMR}\left(\mathrm{CDCl}_{3}, 75 \mathrm{MHz}\right) \delta 14.04,20.86$, 22.68, 28.77, 29.92, 30.94, 31.68, 39.55, 75.92, 129.25, 135.68, 136.18, 136.31, 145.08; IR (neat) $3045 \mathrm{~m}, 2955 \mathrm{~s}, 2924 \mathrm{~s}, 2870 \mathrm{~s}, 1610 \mathrm{~m}, 1466 \mathrm{~s}, 1202 \mathrm{~s} \mathrm{~cm}^{-1}$; mass spectrum $\mathrm{m} / \mathrm{z}(\% \mathrm{rel}$ intensity) $508 \mathrm{M}^{+}$(100), 381 (8), 310 (8), 297 (5), 254 (55), 183 (40), 155 (20), 143 (50), 128 (30).

1,3-Bis-(3-iodoallyl)-2-methoxy-5-methylbenzene $\left(R_{4}=O M e, R_{2}=M e\right) 6 c$ : The pure compound was isolated by silica gel chromatography (20\% dichloromethane/hexanes) in $86 \%$ yield as colorless oil. $R_{f}=0.43$ (hexanes/dichloromethane 4/1). Spectral data for $6 \mathrm{c}:{ }^{1} \mathrm{H}$ NMR $\left(\mathrm{CDCl}_{3}, 300 \mathrm{MHz}\right) \delta 2.26(\mathrm{~s}, 3 \mathrm{H}), 3.35(\mathrm{~d}, 4 \mathrm{H}, \mathrm{J}=6.9 \mathrm{~Hz}), 3.65(\mathrm{~s}, 3 \mathrm{H}), 6.06(\mathrm{dt}, 2 \mathrm{H}, \mathrm{J}=14.0$, $1.5 \mathrm{~Hz}), 6.62-6.67$ (m, $2 \mathrm{H}), 6.88$ (s, $2 \mathrm{H}) ;{ }^{13} \mathrm{C} \mathrm{NMR}\left(\mathrm{CDCl}_{3}, 75 \mathrm{MHz}\right) \delta 20.76,36.16,61.48,76.14$, 129.53, 131.02, 133.94, 144.49, 153.88; IR (neat) 3045m, 2931m, 2828w, 1604m, 1478s, 1232s, 1209 $\mathrm{cm}^{-1}$; mass spectrum $\mathrm{m} / \mathrm{z}$ (\% rel intensity) $454 \mathrm{M}^{+}$(85), 307 (36), 200 (30), 154 (100), calcd for $\mathrm{C}_{14} \mathrm{H}_{16} \mathrm{l}_{2} \mathrm{O} \mathrm{m} / \mathrm{z}$ 453.9291, measd 453.9291 .

3,5-Bis-(3-iodoallyl)-4-methoxybiphenyl $\left(R_{4}=\right.$ OMe, $\left.R_{2}=P h\right) 6 d$ : The pure compound was isolated by silica gel chromatography (20\% dichloromethane/hexanes) in $78 \%$ yield as white solid. $M p=69-71{ }^{\circ} \mathrm{C} . \quad R_{f}=0.33$ (hexanes/dichloromethane 4/1). Spectral data for $6 \mathrm{~d}:{ }^{1} \mathrm{H}$ $\operatorname{NMR}\left(\mathrm{CDCl}_{3}, 300 \mathrm{MHz}\right) 3.32$ (d, $4 \mathrm{H}, \mathrm{J}=6.9 \mathrm{~Hz}$ ), 3.59 (s, $\left.3 \mathrm{H}\right), 5.98$ (d, $2 \mathrm{H}, \mathrm{J}=14.5 \mathrm{~Hz}$ ), $6.52-$ $6.59(\mathrm{~m}, 2 \mathrm{H}), 7.11$ (s, $2 \mathrm{H}), 7.19(\mathrm{t}, 1 \mathrm{H}, \mathrm{J}=6.9 \mathrm{~Hz}), 7.28(\mathrm{t}, 2 \mathrm{H}, \mathrm{J}=7.5 \mathrm{~Hz}), 7.37$ (d, $2 \mathrm{H}, \mathrm{J}=7.2$ $\mathrm{Hz}) ;{ }^{13} \mathrm{C} \mathrm{NMR}\left(\mathrm{CDCl}_{3}, 125 \mathrm{MHz}\right) \delta 36.46,61.59,76.35,127.04,127.26,127.81,128.78,131.82$, 137.71, 140.49, 144.29, 155.79; IR $\left(\mathrm{CH}_{2} \mathrm{Cl}_{2}\right) 3053 \mathrm{w}, 2943 \mathrm{w}, 2828 \mathrm{w}, 1603 \mathrm{w}, 1472 \mathrm{~s}, 1242 \mathrm{~m} \mathrm{~cm}^{-1}$; mass spectrum $m / z$ (\% rel intensity) $516 \mathrm{M}^{+}$(100), 404 (8), 390 (5), 373 (20), 262 (35), 222 (100), 207 (30). Anal. Calcd for $\mathrm{C}_{19} \mathrm{H}_{18} \mathrm{l}_{2} \mathrm{O}: \mathrm{C}, 44.21 ; \mathrm{H}, 3.52$. Found: $\mathrm{C}, 44.51 ; \mathrm{H}, 3.79$.

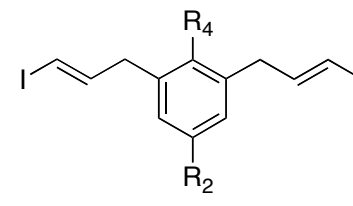

6

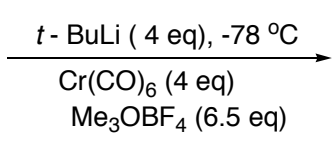

(6.5

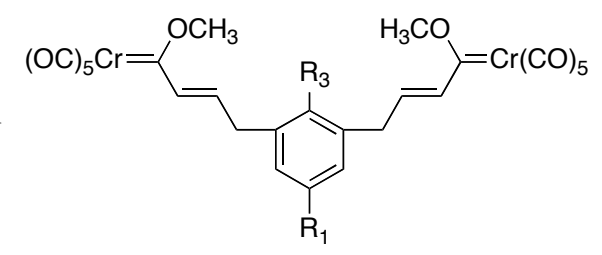

1

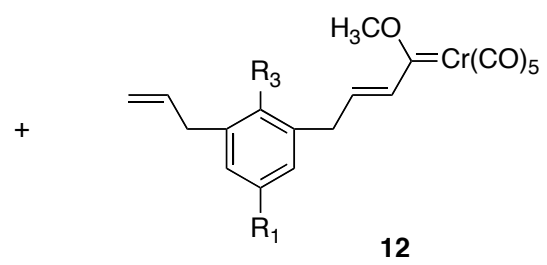

\section{Preparation of the Bis-Carbene complexes 1.}

To a solution of vinyl iodide $6(1 \mathrm{mmol})$ in tetrahydrofuran $(c=0.05 \mathrm{M})$ at $-78{ }^{\circ} \mathrm{C}$ was added $t$-Butyllithium (4 eq) and the reaction mixture was stirred at $-78{ }^{\circ} \mathrm{C}$ for $30 \mathrm{~min}$. Chromium hexacarbonyl (4 eq) was dissolved in $45 \mathrm{~mL}$ of tetrahydrofuran and then transferred via cannula 
to the organolithium solution under argon at $-78{ }^{\circ} \mathrm{C}$. The resulting deep red solution was warmed to room temperature and stirred for $3 \mathrm{~h}$. The solvent was evaporated under vacuum and water/ dichloromethane $(1: 1,50 \mathrm{~mL})$ was added and then trimethyl oxonium tetrafluoroborate (6.5 eq) was added and the mixture stirred for $30 \mathrm{~min}$. The organic layer $(150 \mathrm{~mL})$ was washed with water $(2 \times 50 \mathrm{~mL})$ and dried over anhydrous magnesium sulfate. After filtration the solvent was removed and crude product was purified by silica gel chromatography to give carbene complex as a red oil. In each case a small amount of a less polar product is formed that is tentatively identified by ${ }^{1} \mathrm{H}$ NMR as the mono-carbene complex 12 (5-10\%).

2,6-bis-[2'-propenyl(methoxy)methylene pentacarbonylchromium (0)]-4-methylbiphenyl $\left(R_{3}=P h, R_{1}=M e\right)$ 1a: Following the above procedure, the carbene complex 1a was prepared and purified by silica gel chromatography (hexanes) to give $1 \mathrm{a}$ in $47 \%$ yield as a reddish oil. $R_{f}$ (hexanes) $=0.24$. Spectral data for $1 \mathrm{a}:{ }^{1} \mathrm{H}$ NMR $\left(\mathrm{CDCl}_{3}, 500 \mathrm{MHz}\right) \delta 2.33(\mathrm{~s}, 3 \mathrm{H}), 3.15-3.17(\mathrm{~d}$, $4 \mathrm{H}, \mathrm{J}=6.3 \mathrm{~Hz}$ ), $4.69(\mathrm{~s}, 6 \mathrm{H}), 6.15(\mathrm{dt}, 2 \mathrm{H}, \mathrm{J}=15.0,7.0 \mathrm{~Hz}), 6.97(\mathrm{~s}, 2 \mathrm{H}), 7.05(\mathrm{~d}, 2 \mathrm{H}, \mathrm{J}=15.0$ $\mathrm{Hz}), 7.09$ (d, $2 \mathrm{H}, \mathrm{J}=6.5 \mathrm{~Hz}), 7.33-7.38(\mathrm{~m}, 3 \mathrm{H}) ;{ }^{13} \mathrm{C} \mathrm{NMR}\left(\mathrm{CDCl}_{3}, 75 \mathrm{MHz}\right) \delta 20.93,36.95$, 55.36, 127.48, 128.50, 128.95, 129.46, 134.05, 136.27, 137.87, 138.92, 139.08, 144.45, 216.62, 223.92, 335.86; IR $\left(\mathrm{CH}_{2} \mathrm{Cl}_{2}\right) 3022 \mathrm{w}, 2959 \mathrm{w}, 2924 \mathrm{w}, 2058 \mathrm{~s}, 1921 \mathrm{~s}, 1599 \mathrm{~m}, 1452 \mathrm{~m}, 1226 \mathrm{~m} \mathrm{~cm}^{-1}$; mass spectrum (FAB) $m / z$ (\% rel intensity) $716 \mathrm{M}^{+}$(60), 660 (2), 632 (3), 604 (30), 576 (8), 548 (4), 492 (20), 464 (25), 436 (100), calcd for $\mathrm{C}_{33} \mathrm{H}_{24} \mathrm{Cr}_{2} \mathrm{O}_{12} \mathrm{~m} / \mathrm{z} 716.0078$, measd 716.0077.

1,3-bis-[2'-propenyl(methoxy)methylene pentacarbonylchromium (0)]-2-hexyl-5methylbenzene $\left(R_{3}=C_{6} H_{13}, R_{1}=M e\right) 1 b$ : The general procedure described above was followed and carbene complex $\mathbf{1 b}$ was obtained in $44 \%$ yield as a reddish oil after purification by silica gel chromatography (hexanes). $\mathrm{R}_{\mathrm{f}}$ (hexanes) $=0.3$. Spectral data for $\mathbf{1 b}:{ }^{1} \mathrm{H} \mathrm{NMR}\left(\mathrm{CDCl}_{3}, 500 \mathrm{MHz}\right)$ $\delta 0.87(\mathrm{t}, 3 \mathrm{H}, \mathrm{J}=7.0 \mathrm{~Hz}), 1.34(\mathrm{~m}, 8 \mathrm{H}), 2.23(\mathrm{~s}, 3 \mathrm{H}), 2.49(\mathrm{t}, 2 \mathrm{H}, \mathrm{J}=7.2 \mathrm{~Hz}), 3.44(\mathrm{~d}, 4 \mathrm{H}, \mathrm{J}=$ $6.0 \mathrm{~Hz}$ ), 4.75 (s, $6 \mathrm{H}), 6.31$ (dt, $2 \mathrm{H}, \mathrm{J}=15.5,6.5 \mathrm{~Hz}$ ), $6.84(\mathrm{~s}, 2 \mathrm{H}), 7.23(\mathrm{~d}, 2 \mathrm{H}, \mathrm{J}=15.0 \mathrm{~Hz}$ ); ${ }^{13} \mathrm{C} \mathrm{NMR}\left(\mathrm{CDCl}_{3}, 75 \mathrm{MHz}\right) \delta 14.02,22.62,28.86,29.78,31.21,31.58,35.96,66.44,129.87$, 133.56, 136.07, 136.14, 136.59, 144.63, 216.59, 223.93, 336.02; IR $\left(\mathrm{CH}_{2} \mathrm{Cl}_{2}\right) 2959 w, 2928 w$, $2856 \mathrm{w}, 2060 \mathrm{~s}, 1925 \mathrm{~s}, 1599 \mathrm{~m}, 1425 \mathrm{~m}, 1228 \mathrm{~m} \mathrm{~cm}^{-1}$; mass spectrum (FAB) m/z (\% rel intensity) $724 \mathrm{M}^{+}(3), 668$ (1), 640 (15), 584 (7), 500 (3), 472 (2), 444 (100), calcd for $\mathrm{C}_{33} \mathrm{H}_{32} \mathrm{Cr}_{2} \mathrm{O}_{12} \mathrm{~m} / \mathrm{z}$ 724.0704, measd 724.0707.

1,3-bis-[2'-propenyl(methoxy)methylene pentacarbonylchromium (0)]-2-methoxy-5methylbenzene $\left(R_{3}=\mathrm{OMe}, R_{1}=\mathrm{Me}\right) 1 \mathrm{c}$ : The general procedure as described above was followed and resulting crude carbene complex 1c was purified by silica gel chromatography $(20 \%$ dichloromethane/hexanes) to give $1 \mathrm{c}$ in $36 \%$ yield as a reddish oil. $R_{f}=0.34$ (hexanes/dichloromethane 4/1). Spectral data for 1c: ${ }^{1} \mathrm{H}$ NMR $\left(\mathrm{CDCl}_{3}, 300 \mathrm{MHz}\right) \delta 2.23(\mathrm{~s}, 3 \mathrm{H})$, 3.47 (d, $4 \mathrm{H}, \mathrm{J}=6.0 \mathrm{~Hz}$ ), 3.67 (s, 3 H), $4.72(\mathrm{~s}, 6 \mathrm{H}), 6.33$ (dt, $2 \mathrm{H}, \mathrm{J}=14.0,6.5 \mathrm{~Hz}), 6.85$ (s, $2 \mathrm{H}$ ), $7.32(\mathrm{~d}, 2 \mathrm{H}, \mathrm{J}=14.5 \mathrm{~Hz}) ;{ }^{13} \mathrm{C} \mathrm{NMR}\left(\mathrm{CDCl}_{3}, 75 \mathrm{MHz}\right) \delta 20.60,32.59,61.59,66.47,130.22$, 
$130.74,133.26,134.39,144.77,154.25,216.61,223.92$, 335.96; IR $\left(\mathrm{CH}_{2} \mathrm{Cl}_{2}\right) 2959 \mathrm{w}, 2255 \mathrm{w}$, 2088s, 1934brs, 1603m, 1479w, 1452m, 1228 cm $\mathrm{cm}^{-1}$; mass spectrum (FAB) $\mathrm{m} / \mathrm{z}$ (\% rel intensity) $670 \mathrm{M}^{+}$(1), 530 (8), 446 (7), 418 (8), 390 (14), calcd for $\mathrm{C}_{28} \mathrm{H}_{22} \mathrm{Cr}_{2} \mathrm{O}_{13} \mathrm{~m} / \mathrm{z}$ 669.9871, measd 669.9874.

3,5-bis-[2'-propenyl(methoxy)methylene pentacarbonylchromium (0)]-4-methoxybiphenyl $\left(R_{3}=\mathrm{OMe}, R_{1}=\mathrm{Ph}\right) 1 \mathrm{~d}$ : The general procedure as described above was followed and carbene complex 1d was obtained in $32 \%$ yield as a reddish oil after purification by silica gel chromatography (20\% dichloromethane/hexanes). $R_{f}=0.23$ (hexanes/dichloromethane $=4 / 1$ ). Spectral data for 1d: ${ }^{1} \mathrm{H} \mathrm{NMR}\left(\mathrm{CDCl}_{3}, 500 \mathrm{MHz}\right) \delta 3.56(\mathrm{~d}, 4 \mathrm{H}, \mathrm{J}=7.0 \mathrm{~Hz}), 3.61(\mathrm{~s}, 3 \mathrm{H}), 4.66$ (s, $6 \mathrm{H}), 6.37$ (dt, $2 \mathrm{H}, \mathrm{J}=15.0,7.0 \mathrm{~Hz}$ ), 7.26 (s, $2 \mathrm{H}), 7.31$ (t, $1 \mathrm{H}, \mathrm{J}=7.5 \mathrm{~Hz}), 7.36$ (d, $2 \mathrm{H}, \mathrm{J}=15.0$ $\mathrm{Hz}), 7.39$ (t, $2 \mathrm{H}, \mathrm{J}=7.5 \mathrm{~Hz}), 7.48(\mathrm{~d}, 2 \mathrm{H}, \mathrm{J}=7.5 \mathrm{~Hz}) ;{ }^{13} \mathrm{C} \mathrm{NMR}\left(\mathrm{CDCl}_{3}, 75 \mathrm{MHz}\right) \delta 32.86,61.64$, $66.49,126.99,127.31,128.38,128.75,131.56,132.98,138.03,140.21,144.77,156.01,216.61$, 223.89, 336.02; IR $\left(\mathrm{CH}_{2} \mathrm{Cl}_{2}\right) 2959 \mathrm{w}, 2926 \mathrm{w}, 2060 \mathrm{~s}, 1921 \mathrm{~s}, 1603 \mathrm{~m}, 1473 \mathrm{~m}, 1425 \mathrm{~m}, 1233 \mathrm{~m} \mathrm{~cm}^{-1}$; mass spectrum (FAB) $\mathrm{m} / \mathrm{z}$ (\% rel intensity) $732 \mathrm{M}^{+}$(8), 648 (25), 592 (1), 460 (6), 452 (2), calcd for 732.0027 , measd 732.0038 .

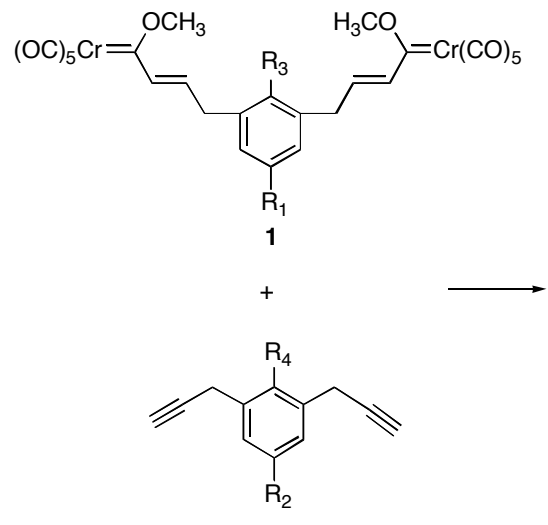

2

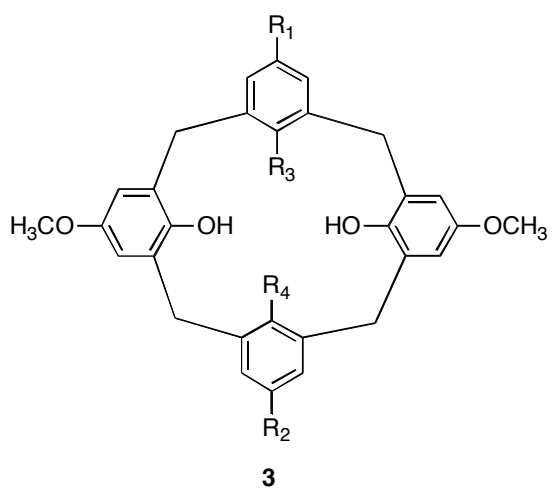

Calixarene Formation by the Triple Annulation of Bis-Carbene Complex 1 with diyne 2.

The bis-carbene complex 1 and the diyne (1:1 molar ratio) were dissolved in 1,2dichloroethane $(2.5 \mathrm{mM})$ in a flame dried $100 \mathrm{~mL}$ or $250 \mathrm{~mL}$ Schlenk flask under Argon. The solution was deoxygenated by freeze pump thaw method in three cycles $\left(-196\right.$ to $\left.25^{\circ} \mathrm{C}\right)$ and then backfilled with argon at ambient temperature. The flask was sealed with a threaded high-vacuum Teflon stopcock and heated to $100{ }^{\circ} \mathrm{C}$ for $20-40$ min during which time the deep red solution turned yellow. The yellow solution was stirred overnight exposed to air to facilitate de-metalation of the arenechromium tricarbonyl complex. The solvent was removed under vacuum and the residue dissolved in ethyl acetate $(50 \mathrm{~mL})$ and then filtered through a short pad of silica gel. 
Further washing of the $\mathrm{SiO}_{2}$ pad with ethyl acetate and evaporation of the solvent gave the crude calixarene which was purified by flash chromatography on silica gel.

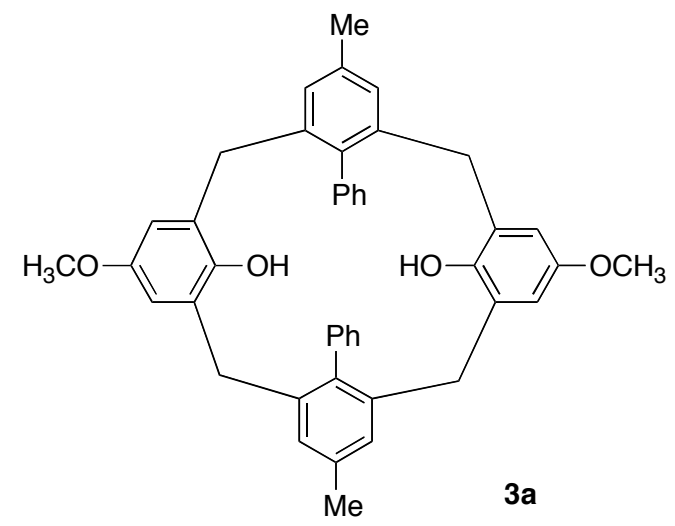

5,17-dimethyl-11,23-dimethoxy-25,27-dihydroxy-26,28-diphenylcalix(4)arene 3a: The bis-carbene complex 1a $(0.121 \mathrm{~g}, 0.165 \mathrm{mmol})$ and diyne $2 \mathrm{a}(0.0403 \mathrm{~g}, 0.165 \mathrm{mmol})$ were subjected to the benzannulation reaction in $66 \mathrm{~mL}$ of 1,2-dichloroethane following the general procedure and afforded a separable $1.0: 1.7$ mixture of two conformers 3a-I and 3a-II which were separated by silica gel chromatography ( $10 \%$ ethyl acetate / hexanes) and obtained as solids in $13 \%$ and $22 \%$ yields, respectively. Each was crystallized from dichloromethane/hexanes to give single crystals, which upon X-ray diffraction analysis, revealed that the minor isomer 3a-I exists as a pinched cone conformation and the major isomer 3a-II exists as a pinched 1,2-alternate conformation. The following physical and spectral data were collected for the two conformers.

Conformer 3a-I: $R_{\mathrm{f}}=0.37$ (hexanes/ ethyl acetate $\left.=9 / 1\right) \mathrm{Mp}=221-224{ }^{\circ} \mathrm{C} .{ }^{1} \mathrm{H}$ NMR $\left(\mathrm{CDCl}_{3}\right.$, $500 \mathrm{MHz}) \delta 2.01$ (s, $6 \mathrm{H}$ ), 3.33 (d, $4 \mathrm{H}, \mathrm{J}=14.5 \mathrm{~Hz}$ ), 3.74 (d, $4 \mathrm{H}, \mathrm{J}=15.5 \mathrm{~Hz}$ ), 3.77 (s, $6 \mathrm{H}$ ), 4.24 (s, $2 \mathrm{H}, \mathrm{OH}), 6.51(\mathrm{~s}, 4 \mathrm{H}), 6.55$ (s, $4 \mathrm{H}), 6.98(\mathrm{~d}, 2 \mathrm{H}, \mathrm{J}=6.5 \mathrm{~Hz}), 7.35-7.38(\mathrm{~m}, 4 \mathrm{H}), 7.50-7.52$ $(\mathrm{m}, 2 \mathrm{H}), 8.14(\mathrm{~d}, 2 \mathrm{H}, \mathrm{J}=8.0 \mathrm{~Hz}) ;{ }^{13} \mathrm{C} \mathrm{NMR}\left(\mathrm{CDCl}_{3}, 75 \mathrm{MHz}\right) \delta 20.89$, 36.12, 55.68, 114.44, $126.99,128.14,130.32,131.73,132.15,136.89,138.15,138.87,139.27,146.78,152.72$; IR $\left(\mathrm{CH}_{2} \mathrm{Cl}_{2}\right) 3472 \mathrm{br}, 3056 \mathrm{~s}, 2988 \mathrm{~m}, 1635 \mathrm{~m}, 1610 \mathrm{~m}, 1479 \mathrm{~m}, 1412 \mathrm{~m}, 12656 \mathrm{~s}, 1145 \mathrm{w} \mathrm{cm}{ }^{-1}$; mass spectrum $\mathrm{m} / \mathrm{z}$ (\% rel intensity) $632 \mathrm{M}^{+}$(100), 315 (30), 193 (10), 179 (15), 149 (20), calcd for $\mathrm{C}_{44} \mathrm{H}_{40} \mathrm{O}_{4} \mathrm{~m} / \mathrm{z}$ 632.2927, measd 632.2924. Conformer 3a-II: $\mathrm{R}_{\mathrm{f}}=0.45$ (hexanes/ ethyl acetate $=$ 9/1) $\mathrm{Mp}=260-262{ }^{\circ} \mathrm{C} .{ }^{1} \mathrm{H}$ NMR $\left(\mathrm{CDCl}_{3}, 500 \mathrm{MHz}\right) \delta 2.36(\mathrm{~s}, 6 \mathrm{H}), 3.53(\mathrm{~d}, 4 \mathrm{H}, \mathrm{J}=15.0 \mathrm{~Hz}), 3.61$ (d, $4 \mathrm{H}, \mathrm{J}=14.5 \mathrm{~Hz}$ ), 3.66 (s, $6 \mathrm{H}$ ), $4.12(\mathrm{~s}, 2 \mathrm{H}, \mathrm{OH}), 6.26$ (d, $2 \mathrm{H}, \mathrm{J}=7.5 \mathrm{~Hz}), 6.35$ (s, $4 \mathrm{H}), 6.82$ (t, $2 \mathrm{H}, \mathrm{J}=7.8 \mathrm{~Hz}$ ), 6.97 (d, $2 \mathrm{H}, \mathrm{J}=7.5 \mathrm{~Hz}$ ), 7.10 (s, $4 \mathrm{H}), 7.16(\mathrm{t}, 2 \mathrm{H}, \mathrm{J}=7.3 \mathrm{~Hz}), 7.25(\mathrm{~m}, 2 \mathrm{H})$; ${ }^{13} \mathrm{C}$ NMR $\left(\mathrm{CDCl}_{3}, 75 \mathrm{MHz}\right) \delta 21.02,36.95,55.59,113.76,126.48,127.15,128.87,129.24$, 130.00, 130.78, 137.28, 138.83, 139.70, 147.34, 152.16; IR $\left(\mathrm{CH}_{2} \mathrm{Cl}_{2}\right) 3509 \mathrm{~s}, 3052 \mathrm{w}, 3005 \mathrm{w}$, 2918w, 2837w, 1590w, 1481s, 1441m, 1244m, 1149m, 1057m cm $\mathrm{cm}^{-1}$. 


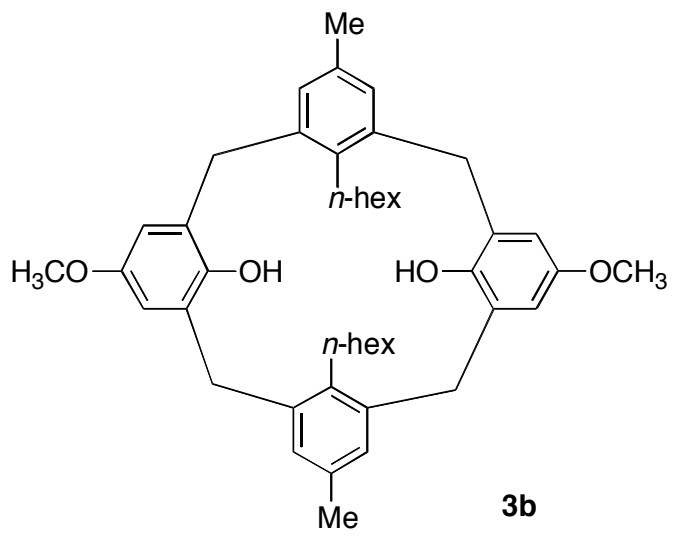

5,17-dimethyl-11,23-dimethoxy-25,27-dihydroxy-26,28-dihexylcalix(4)arene $3 \mathrm{~b}$ : $\mathrm{A}$ solution of the bis-carbene complex $\mathbf{1 b}(0.221 \mathrm{~g}, 0.306 \mathrm{mmol})$ and diyne $\mathbf{2 b}(0.082 \mathrm{~g}, 0.32 \mathrm{mmol})$ in $120 \mathrm{~mL}$ of 1,2-dichloroethane was subjected to the reaction conditions described above and the resulting calixarene $\mathbf{3 b}$ was purified by silica gel chromatography (5\% ethyl acetate/hexanes) to give a $22 \%$ yield of $\mathbf{3 b}$ as white solid and as a single conformation, whose structure was not assigned. $R_{f}=0.39$ (hexanes / ethyl acetate $=19 / 1$ ) $M p=158-160{ }^{\circ} \mathrm{C}$. Spectral data for $\mathbf{3 b}:{ }^{1} \mathrm{H}$ NMR $\left(\mathrm{CDCl}_{3}, 500 \mathrm{MHz}\right) \delta 0.82(\mathrm{t}, 6 \mathrm{H}, \mathrm{J}=7.3 \mathrm{~Hz}), 1.15-1.23(\mathrm{~m}, 16 \mathrm{H}), 2.21(\mathrm{~s}, 6 \mathrm{H}), 2.52(\mathrm{t}, 4 \mathrm{H}$, $\mathrm{J}=7.5 \mathrm{~Hz}$ ), $3.62(\mathrm{~d}, 4 \mathrm{H}, \mathrm{J}=15.0 \mathrm{~Hz}), 3.81(\mathrm{~s}, 6 \mathrm{H}), 3.91(\mathrm{~s}, 2 \mathrm{H}), 4.05(\mathrm{~d}, 4 \mathrm{H}, \mathrm{J}=15.0 \mathrm{~Hz}), 6.66$ (s, $4 \mathrm{H}), 6.96$ (s, $4 \mathrm{H}) ;{ }^{13} \mathrm{C}$ NMR $\left(\mathrm{CDCl}_{3}, 75 \mathrm{MHz}\right) \delta 14.04,20.89,22.59,27.73,29.29,31.67$, $31.81,36.86,55.69,113.86,128.53,130.32,135.57,137.87,138.63,147.49,152.66$; IR $\left(\mathrm{CH}_{2} \mathrm{Cl}_{2}\right) 3499 \mathrm{~m}, 2924 \mathrm{~s}, 2855 \mathrm{~s}, 1606 \mathrm{w}, 1466 \mathrm{~m}, 1377 \mathrm{w}, 1246 \mathrm{w}, 1147 \mathrm{w}, 1059 \mathrm{w} \mathrm{cm}{ }^{-1}$.

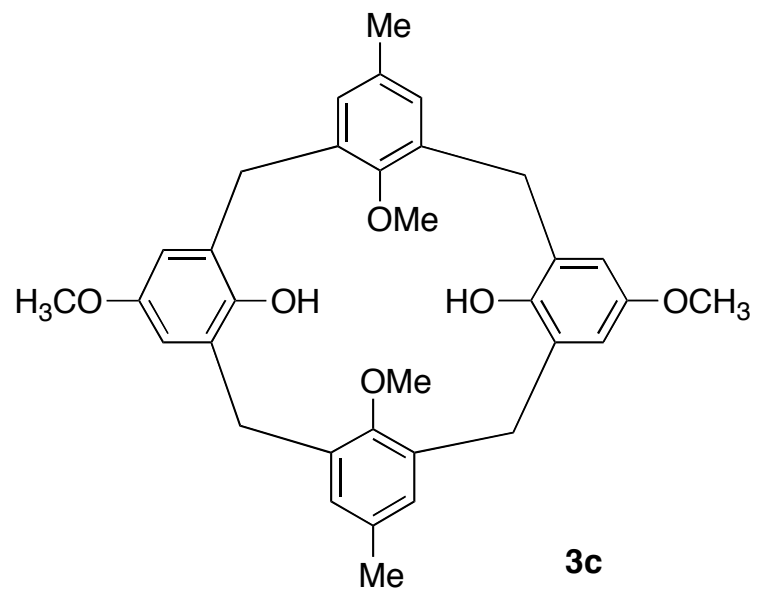

5,17-dimethyl-11,23,26,28-dimethoxy-25,27-dihydroxycalix(4)arene 3c: The bis-carbene complex 1c $(0.188 \mathrm{~g}, 0.28 \mathrm{mmol})$ and diyne $1 \mathrm{c}(0.055 \mathrm{~g}, 0.28 \mathrm{mmol})$ were dissolved in $112 \mathrm{~mL}$ of 1,2-dichloroethane and subjected to the reaction conditions described above which gave the calixarene $\mathbf{3 c}$ in $36 \%$ yield as a white solid and as a single conformer after purification by silica 
gel chromatography (25\% ethyl acetate/hexanes). This compound was crystallized from acetonitrile and subjected to a single crystal X-ray diffraction analyses which revealed it to be the cone conformer of $3 \mathbf{c}$ (see below). $\mathrm{Mp}>298^{\circ} \mathrm{C}$ with decomposition. $\mathrm{R}_{\mathrm{f}}=0.32$ (hexanes/ ethyl acetate = 3/1). Spectral data for 3c: ${ }^{1} \mathrm{H} \mathrm{NMR}\left(\mathrm{CDCl}_{3}, 300 \mathrm{MHz}\right) \delta 2.03(\mathrm{~s}, 6 \mathrm{H}), 3.27(\mathrm{~d}, 4 \mathrm{H}, \mathrm{J}=$ $13.2 \mathrm{~Hz}$ ), 3.74 (s, $6 \mathrm{H}$ ), 3.93 (s, $6 \mathrm{H}$ ), 4.27 (d, $4 \mathrm{H}, \mathrm{J}=12.9 \mathrm{~Hz}$ ), 6.61 (s, $4 \mathrm{H}), 6.72(\mathrm{~s}, 4 \mathrm{H}), 7.59$ $(\mathrm{s}, 2 \mathrm{H}, \mathrm{OH}) ;{ }^{13} \mathrm{C} \mathrm{NMR}\left(\mathrm{CDCl}_{3}, 75 \mathrm{MHz}\right) \delta 20.86,31.53,55.78,63.49,113.72,129.12,129.69$, 132.74, 134.32, 146.91, 151.30, 152.19; IR $\left(\mathrm{CH}_{2} \mathrm{Cl}_{2}\right) 3297 \mathrm{br}, 3055 \mathrm{w}, 2988 \mathrm{w}, 2937 \mathrm{w}, 2835 \mathrm{w}$, $1600 \mathrm{w}, 1481 \mathrm{~s}, 1433 \mathrm{~m}, 1285 \mathrm{~s}, 1228 \mathrm{~m}, 1124 \mathrm{~m}, 1055 \mathrm{~m}, 1009 \mathrm{~m} \mathrm{~cm}^{-1}$; Mass spectrum, calcd for $\mathrm{C}_{34} \mathrm{H}_{36} \mathrm{O}_{6} \mathrm{~m} / \mathrm{z} 540.2512$, measd 540.2512. Anal calcd for $\mathrm{C}_{34} \mathrm{H}_{36} \mathrm{O}_{6}: \mathrm{C}, 75.53 ; \mathrm{H}, 6.71$. Found: $\mathrm{C}$, $75.62 ; \mathrm{H}, 6.60$.

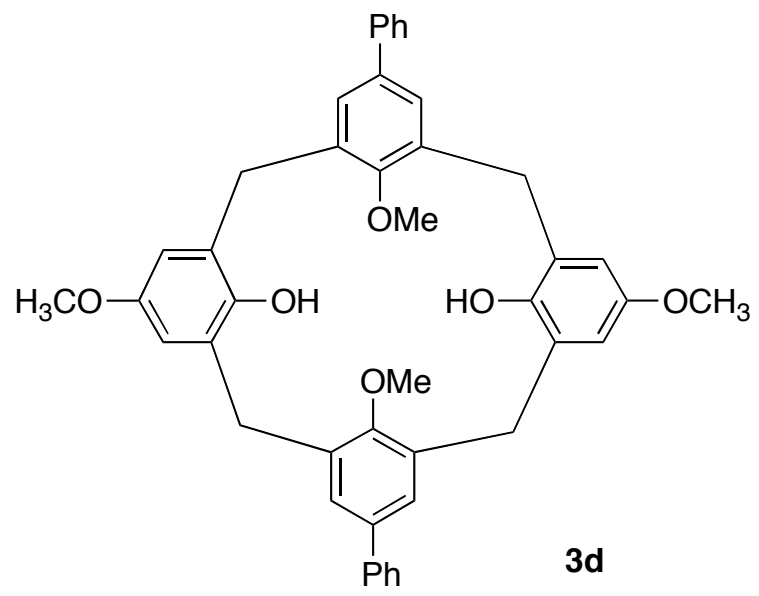

5,17-diphenyl-11,23,26,28-tetramethoxy-25,27-dihydroxycalix(4)arene $3 \boldsymbol{d}$ : A solution of the bis-carbene complex $1 \mathbf{d}(0.105 \mathrm{~g}, 0.145 \mathrm{mmol})$ and diyne $2 \mathrm{~d}(0.038 \mathrm{~g}, 0.145 \mathrm{mmol})$ in $58 \mathrm{~mL}$ of 1,2-dichloroethane was subjected to the benzannulation conditions described above and the calixarene 3d was isolated as a white solid in $41 \%$ yield as a single conformation after purification by silica gel chromatography (25\% EtOAc/hexanes). $\mathrm{Mp}=276-279{ }^{\circ} \mathrm{C}$ with decomposition. $R_{f}=0.25$ (hexanes/ethylacetate $=3 / 1$ ). The conformation of $\mathbf{3 d}$ was tentatively assigned as the cone conformation based on the chemical shift of the phenol hydrogens $(\delta=$ 7.36). The chemical shift of the phenol protons of $3 c$ (cone conformer) is $\delta=7.59$, whereas, the chemical shift of the phenol protons of the partial cone of $3 e$ is $\delta=5.75$. The chemical shifts of the phenol hydrogens of a 1,3 -alternate conformer of a related calixarene is $\delta=4.01 .^{8}$ Spectral data for 3d: ${ }^{1} \mathrm{H}$ NMR $\left(\mathrm{CDCl}_{3}, 500 \mathrm{MHz}\right) \delta 3.39(\mathrm{~d}, 4 \mathrm{H}, \mathrm{J}=13.0 \mathrm{~Hz}), 3.73(\mathrm{~s}, 6 \mathrm{H}), 4.01(\mathrm{~s}, 6 \mathrm{H})$, 4.38 (d, $4 \mathrm{H}, \mathrm{J}=13.0 \mathrm{~Hz}$ ), 5.28 (s, $2 \mathrm{H}$ ), 6.66 (s, $4 \mathrm{H}$ ), 7.09 (s, $4 \mathrm{H})$, 7.16-7.21 (m, $3 \mathrm{H})$, 7.24-7.25 $(\mathrm{m}, 5 \mathrm{H}), 7.36(\mathrm{~s}, 2 \mathrm{H}, \mathrm{OH}) ;{ }^{13} \mathrm{C} \mathrm{NMR}\left(\mathrm{CDCl}_{3}, 125 \mathrm{MHz}\right) \delta 31.73,55.85,63.54,113.93,126.86$, $126.95,127.85,128.45,129.09,133.41,137.96,140.56,146.9,152.48,153.25 ;$ IR $\left(\mathrm{CH}_{2} \mathrm{Cl}_{2}\right)$ $3327 \mathrm{br}, 2934 \mathrm{w}, 2829 \mathrm{w}, 1483 \mathrm{~s}, 1431 \mathrm{~s}, 1234 \mathrm{~m}, 1138 \mathrm{~m}, 1003 \mathrm{~m}, 906 \mathrm{~m} \mathrm{~cm}^{-1}$; mass spectrum $\mathrm{m} / \mathrm{z}$ 
(\% rel intensity) 664 (100), 633 (5), 602 (40), 332 (15), 301 (10); calcd for $\mathrm{C}_{44} \mathrm{H}_{40} \mathrm{O}_{6} \mathrm{~m} / \mathrm{z}$ 664.2825 , measd 664.2825.

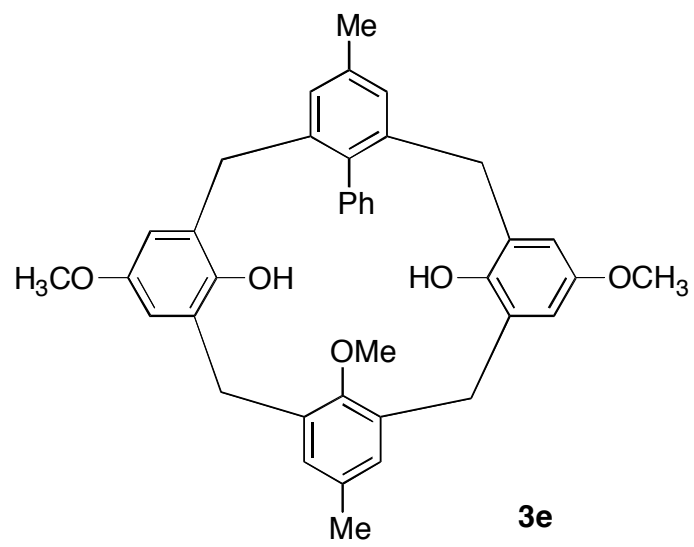

5,17-dimethyl-11,23,26-trimethoxy-25,27-dihydroxy-28 phenylcalix(4)arene $3 \mathbf{e}$ : The biscarbene complex 1c $(0.124 \mathrm{~g}, 0.185 \mathrm{mmol})$ and diyne $2 \mathrm{a}(0.045 \mathrm{~g}, 0.185 \mathrm{mmol})$ in $74 \mathrm{~mL}$ of $1,2-$ dichloroethane were reacted according to the procedure described above to give the calixarene 3e as a 3.8:1 mixture of inseparable conformers in $31 \%$ yield as a white solid after purification by silica gel chromatography (25\% EtOAc/hexanes). $M p=240-242{ }^{\circ} \mathrm{C} . \mathrm{R}_{\mathrm{f}}$ (hexanes/ethylacetate $3 / 1)=0.44$. HPLC analysis showed the presence of a single peak at 10.19 min upon gradient elution with a hexane/iso-propanol mixture of that was varied from $99.5 / 0.5$ to $97 / 3$ over $40 \mathrm{~min}$ at a flow rate of $1 \mathrm{~mL} / \mathrm{min}$ using a silica gel column (R0086100C5). The ${ }^{1} \mathrm{H} N M R$ in $\mathrm{CDCl}_{3}$ showed the presence of two conformers in a ratio of 3.8:1 as measured by integration of the peaks at $\delta=$ 2.02 and 2.45. The different ratio of isomers in different solvents shows that these conformers do equilibrate rapidly but not on the NMR time scale (Table 3). Also, variable temperature ${ }^{1} \mathrm{H}$ NMR was recorded from 25 to $95^{\circ} \mathrm{C}$ in DMSO- $\mathrm{d}_{6}$ but no coalescence of peaks was observed. EXSY experiments at $50{ }^{\circ} \mathrm{C}(\mathrm{t} \mathrm{mix}=0.7 \mathrm{~s}, \mathrm{ni}=64, \mathrm{nt}=256$, threefold forward linear prediction along $\mathrm{F} 1$ dimension) reveal that rotation about the phenyl group and interconversion between conformers is rapid but not on the NMR time scale (Figure 1). On the basis of NOE experiments the major conformer was assigned as a partial cone and the minor as the cone conformation (see below). The mass spectrum of $3 e$ shows a trace peak at $\mathrm{m} / \mathrm{z}=1172(0.13)$. This is attributed to a trace of a calix[8]arene that can not be detected by ${ }^{1} \mathrm{H}$ NMR. The two conformers observed by ${ }^{1} \mathrm{H}$ NMR are shown to be interconverting by the solvent experiments and EXSY experiments shown below. A calix[8]arene would not be expected to exists as conformers that could be observed on the ${ }^{1} \mathrm{H}$ NMR time scale. The following spectral data were obtained on a mixture of the two conformers. Spectral data for $3 e:{ }^{1} \mathrm{H}$ NMR $\left(\mathrm{CDCl}_{3}, 500 \mathrm{MHz}\right)$ major; $\delta 2.25(\mathrm{~s}, 3 \mathrm{H}), 2.43(\mathrm{~s}, 3 \mathrm{H}), 3.25(\mathrm{~d}, 2 \mathrm{H}$, $\mathrm{J}=13.5 \mathrm{~Hz}) 3.46(\mathrm{~s}, 6 \mathrm{H}), 3.49(\mathrm{~d}, 2 \mathrm{H}, \mathrm{J}=13.0 \mathrm{~Hz}), 3.84(\mathrm{~s}, 3 \mathrm{H}), 3.88(\mathrm{~d}, 2 \mathrm{H}, \mathrm{J}=13 \mathrm{~Hz}$ 
overlapping with peak at $\delta$ 3.84), $4.11(\mathrm{~d}, 2 \mathrm{H}, \mathrm{J}=13.0 \mathrm{~Hz}), 4.78(\mathrm{~d}, 1 \mathrm{H}, \mathrm{J}=7.5 \mathrm{~Hz}), 5.51(\mathrm{t}, 1 \mathrm{H}$, $\mathrm{J}=7.5 \mathrm{~Hz}$ ), $5.64(\mathrm{~d}, 2 \mathrm{H}, \mathrm{J}=3.0 \mathrm{~Hz}), 5.75(\mathrm{~s}, 2 \mathrm{H}, \mathrm{OH}), 6.48(\mathrm{~d}, 2 \mathrm{H}, \mathrm{J}=3.5 \mathrm{~Hz}), 6.67(\mathrm{t}, 2 \mathrm{H}, \mathrm{J}=$ $6.5 \mathrm{~Hz}$ ), $6.83(\mathrm{~d}, 1 \mathrm{H}, \mathrm{J}=7.5 \mathrm{~Hz}), 6.90(\mathrm{~s}, 2 \mathrm{H}), 7.03(\mathrm{t}, 1 \mathrm{H}, \mathrm{J}=7.5 \mathrm{~Hz}), 7.23(\mathrm{~s}, 2 \mathrm{H})$; minor; $\delta$ $1.81(\mathrm{~s}, 3 \mathrm{H}), 2.02(\mathrm{~s}, 3 \mathrm{H}), 3.29(\mathrm{~d}, 2 \mathrm{H}, \mathrm{J}=13.5 \mathrm{~Hz}$ overlapping with peak at $\delta 3.26$ of major isomer), $3.35(\mathrm{~d}, 2 \mathrm{H}, \mathrm{J}=14.0 \mathrm{~Hz}), 3.73(\mathrm{~d}, 2 \mathrm{H}, \mathrm{J}=14.0 \mathrm{~Hz}), 3.78(\mathrm{~s}, 6 \mathrm{H}), 3.86(\mathrm{~s}, 3 \mathrm{H}), 4.11(\mathrm{~d}$, $2 \mathrm{H}, \mathrm{J}=14.0 \mathrm{~Hz}), 5.01(\mathrm{~s}, 2 \mathrm{H}), 6.34(\mathrm{~s}, 2 \mathrm{H}), 6.51(\mathrm{~d}, 2 \mathrm{H}, \mathrm{J}=2.5 \mathrm{~Hz}), 6.58(\mathrm{~s}, 2 \mathrm{H}), 6.65(\mathrm{~d}, 2 \mathrm{H}$, $\mathrm{J}=3 \mathrm{~Hz}), 7.29-7.33(\mathrm{~m}, 1 \mathrm{H}), 7.41(\mathrm{t}, 2 \mathrm{H}, \mathrm{J}=7.5 \mathrm{~Hz}), 7.92(\mathrm{~d}, 2 \mathrm{H}, \mathrm{J}=8.0 \mathrm{~Hz}) ;{ }^{13} \mathrm{C} \mathrm{NMR}$ $\left(\mathrm{CDCl}_{3}, 125 \mathrm{MHz}\right)$ major; $\delta$ 20.86, 21.15, 31.12, 40.10, 55.32, 63.16, 113.03, 113.99, 125.19, 125.97, 126.61, 126.90, 128.12, 129.61, 129.96, 130.06, 130.40, 133.54, 134.78, 137.51, 138.59, 139.34, 146.61, 152.56, minor; $\delta 25.60,29.50$, 32.04, 36.24, 55.69, 63.02, 113.78, 114.50, 127.52, 127.70, 127.83, 129.49, 130.92, 131.15, 132.06, 132.11, 134.10, 136.20, 138.90, 139.01, 139.60, 147.11, 151.81, 152.40; IR $\left(\mathrm{CH}_{2} \mathrm{Cl}_{2}\right) 3499 \mathrm{br}, 3055 \mathrm{~m}, 2988 \mathrm{w}, 1606 \mathrm{w}, 1481 \mathrm{~m}, 1468 \mathrm{w}$, 1421w, 1265s, 1055 $\mathrm{cm}^{-1}$; mass spectrum (FAB MS in 4-nitrobenzyl alcohol] $\mathrm{m} / \mathrm{z}$ (\% rel intensity) $586 \mathrm{M}^{+}$(100), 307 (10), 154 (37), 137 (26), 77 (6), calcd for $\mathrm{C}_{39} \mathrm{H}_{38} \mathrm{O}_{5} \mathrm{~m} / z$ 586.2719, measd 586.2715. Anal Calcd for $\mathrm{C}_{39} \mathrm{H}_{38} \mathrm{O}_{5}$ : $\mathrm{C}, 79.84 ; \mathrm{H}, 6.53$. Found: $\mathrm{C}, 80.04 ; \mathrm{H}, 6.22$.

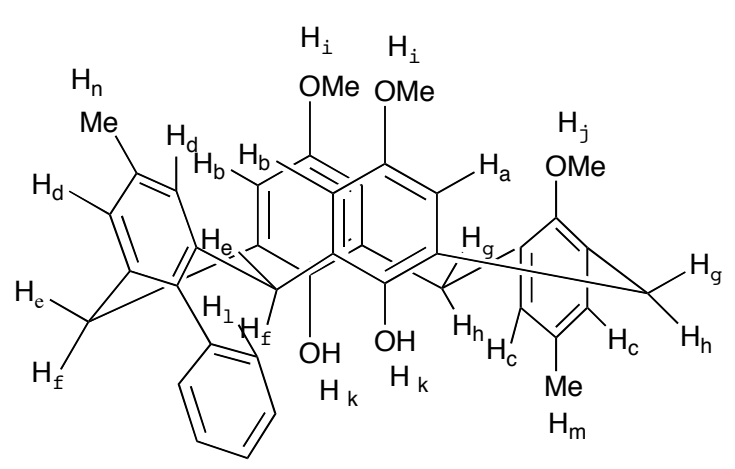

3e Partial Cone conformation

\begin{tabular}{|cc|}
\hline $\begin{array}{c}\text { Chemical Shifts For } \mathbf{H}_{\mathrm{a}}-\mathbf{H}_{\mathbf{n}} \text { Major } \\
\delta \text { ppm }\end{array}$ \\
\hline $\mathrm{H}_{\mathrm{a}}=5.64(\mathrm{~d})$ & $\mathrm{H}_{\mathrm{j}}=3.84(\mathrm{~s})$ \\
$\mathrm{H}_{\mathrm{b}}=6.49(\mathrm{~d})$ & $\mathrm{H}_{\mathrm{k}}=5.75(\mathrm{~s})$ \\
$\mathrm{H}_{\mathrm{c}}=7.23(\mathrm{~s})$ & $\mathrm{H}_{\mathrm{l}}=4.78(\mathrm{~d})$ \\
$\mathrm{H}_{\mathrm{d}}=6.90(\mathrm{~s})$ & $\mathrm{H}_{\mathrm{m}}=2.43(\mathrm{~s})$ \\
$\mathrm{H}_{\mathrm{e}}=3.25(\mathrm{~d})$ & $\mathrm{H}_{\mathrm{n}}=2.25(\mathrm{~s})$ \\
$\mathrm{H}_{\mathrm{f}}=4.11(\mathrm{~d})$ & \\
$\mathrm{H}_{\mathrm{g}}=3.49(\mathrm{~d})$ & \\
$\mathrm{H}_{\mathrm{h}}=3.88(\mathrm{~d})$ & \\
$\mathrm{H}_{\mathrm{i}}=3.46(\mathrm{~s})$ & \\
\hline
\end{tabular}


Table 1 Results of Homonuclear ${ }^{1} \mathrm{H}-{ }^{1} \mathrm{H}$ NOE experiments on Major isomer of $3 \mathbf{e}$ (Partial cone).

\begin{tabular}{|c|c|c|c|c|c|}
\hline Nuclei Irradiated & Adjacent $\mathrm{H}$ & Enhancement & Nuclei irradiated & Adjacent $\mathrm{H}$ & Enhancement \\
\hline \multirow[t]{2}{*}{$5.64\left(\mathrm{H}_{\mathrm{a}}\right)$} & $\mathrm{H}_{\mathrm{g}}$ & $y$ & $4.11\left(\mathrm{H}_{\mathrm{f}}\right)$ & $\mathrm{H}_{\mathrm{e}}$ & y \\
\hline & $\mathrm{H}_{\mathrm{i}}$ & y & & $\mathrm{H}_{\mathrm{k}}$ & $y$ \\
\hline \multirow[t]{3}{*}{$6.49\left(\mathrm{H}_{\mathrm{b}}\right)$} & $\mathrm{H}_{\mathrm{d}}$ & $y$ & $3.49\left(\mathrm{H}_{\mathrm{g}}\right)$ & $\mathrm{H}_{\mathrm{h}}$ & $\mathrm{y}$ \\
\hline & $\mathrm{H}_{\mathrm{i}}$ & $y$ & & $\mathrm{H}_{\mathrm{a}}$ & $\mathrm{y}$ \\
\hline & $\mathrm{H}_{\mathrm{e}}$ & y & & $\mathrm{H}_{\mathrm{j}}$ & $y$ \\
\hline \multirow[t]{3}{*}{ 7.23( $\left.\mathrm{H}_{\mathrm{c}}\right)$} & $\mathrm{H}_{\mathrm{h}}$ & $\mathrm{y}$ & $3.88\left(\mathrm{H}_{h}\right)$ & $\mathrm{H}_{\mathrm{g}}$ & $y$ \\
\hline & $\mathrm{H}_{\mathrm{m}}$ & $y$ & & $\mathrm{H}_{\mathrm{c}}$ & y \\
\hline & $\mathrm{H}_{\mathrm{k}}$ & y & $3.46\left(\mathrm{H}_{\mathrm{i}}\right)$ & $\mathrm{H}_{\mathrm{a}}$ & $\mathrm{y}$ \\
\hline \multirow[t]{3}{*}{$6.90\left(\mathrm{H}_{\mathrm{d}}\right)$} & $\mathrm{H}_{\mathrm{b}}$ & y & $3.84\left(\mathrm{H}_{j}\right)$ & $\mathrm{H}_{\mathrm{g}}$ & $\mathrm{y}$ \\
\hline & $\mathrm{H}_{\mathrm{e}}$ & $y$ & $5.75\left(\mathrm{H}_{\mathrm{k}}\right)$ & $\mathrm{H}_{\mathrm{f}}$ & $y$ \\
\hline & $\mathrm{H}_{\mathrm{n}}$ & y & & $\mathrm{H}_{\mathrm{h}}$ & $y$ \\
\hline \multirow[t]{4}{*}{$3.25\left(\mathrm{H}_{\mathrm{e}}\right)$} & $\mathrm{H}_{\mathrm{f}}$ & $y$ & & $\mathrm{H}_{1}$ & $\mathrm{y}$ \\
\hline & $\mathrm{H}_{\mathrm{d}}$ & $y$ & & $\mathrm{H}_{\mathrm{c}}$ & $y$ \\
\hline & $\mathrm{H}_{\mathrm{b}}$ & y & $4.78\left(\mathrm{H}_{1}\right)$ & $\mathrm{H}_{\mathrm{k}}$ & $y$ \\
\hline & & & & Meta $(\mathrm{Ar}-\mathrm{H})$ & $y$ \\
\hline
\end{tabular}




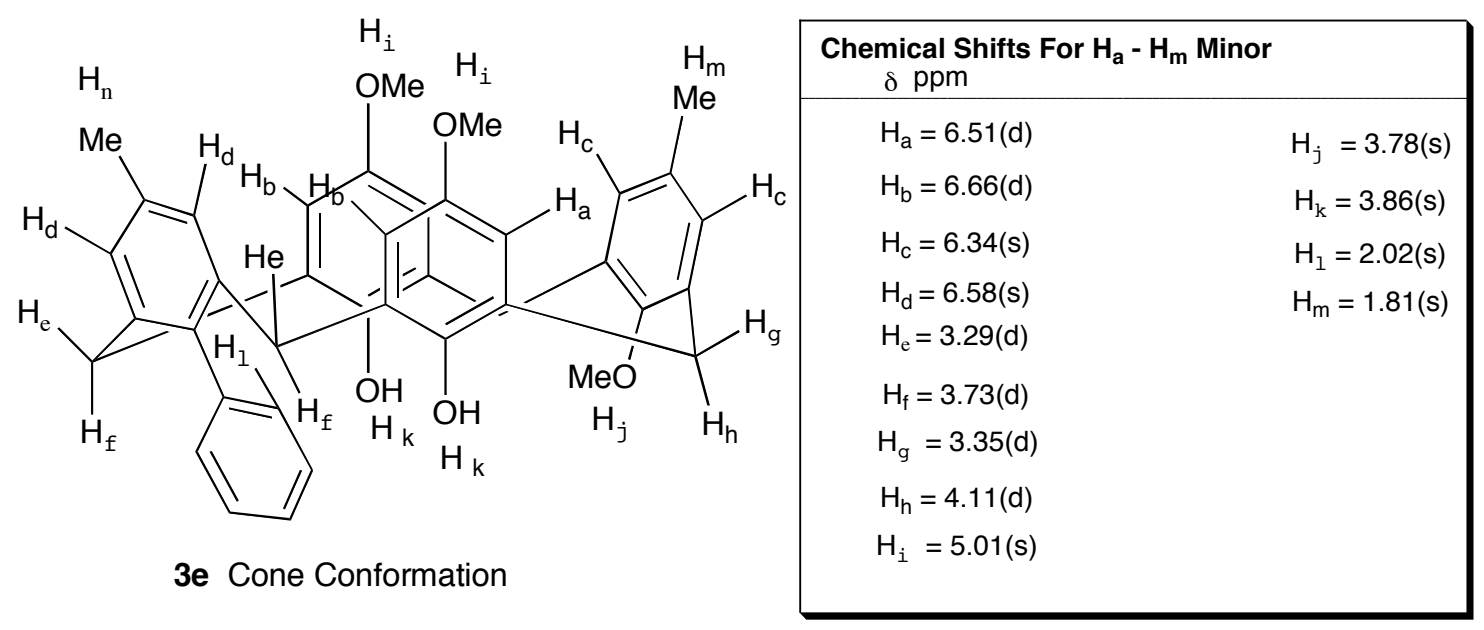

Table 2 Results of Homonuclear ${ }^{1} \mathrm{H}-{ }^{1} \mathrm{H}$ NOE experiments on Minor Isomer of $3 \mathbf{e}$ (Cone).

\begin{tabular}{|c|c|c|c|c|c|}
\hline Nuclei irradiated & Adjacent $\mathrm{H}$ & Enhancement & Nuclei irradiated & Adjacent $\mathrm{H}$ & Enhancement \\
\hline \multirow[t]{3}{*}{$6.51\left(\mathrm{H}_{\mathrm{a}}\right)$} & $\mathrm{H}_{\mathrm{j}}$ & $y$ & & $\mathrm{H}_{\mathrm{d}}$ & $y$ \\
\hline & $\mathrm{H}_{\mathrm{e}}$ & $y$ & $4.11\left(\mathrm{H}_{\mathrm{h}}\right)$ & $\mathrm{H}_{\mathrm{g}}$ & $y$ \\
\hline & $\mathrm{H}_{\mathrm{c}}$ & $y$ & & $\mathrm{H}_{\mathrm{k}}$ & $y$ \\
\hline \multirow[t]{2}{*}{$6.65\left(\mathrm{H}_{\mathrm{b}}\right)$} & $\mathrm{H}_{\mathrm{j}}$ & $y$ & & $\mathrm{H}_{\mathrm{i}}$ & $\mathrm{y}$ \\
\hline & $\mathrm{H}_{\mathrm{g}}$ & $y$ & $5.01\left(\mathrm{H}_{\mathrm{i})}\right.$ & $\mathrm{H}_{\mathrm{f}}$ & y \\
\hline \multirow[t]{3}{*}{$6.34\left(\mathrm{H}_{\mathrm{c}}\right)$} & $\mathrm{H}_{\mathrm{a}}$ & $\mathrm{y}$ & $3.78\left(\mathrm{H}_{j}\right)$ & $\mathrm{Ha}$ & $y$ \\
\hline & $\mathrm{H}_{\mathrm{e}}$ & $y$ & & $\mathrm{H}_{\mathrm{b}}$ & $\mathrm{y}$ \\
\hline & $\mathrm{H}_{\mathrm{m}}$ & $y$ & & & \\
\hline \multirow[t]{3}{*}{$6.58\left(\mathrm{H}_{\mathrm{d}}\right)$} & $\mathrm{H}_{\mathrm{b}}$ & $\mathrm{y}$ & $3.86\left(\mathrm{H}_{\mathrm{k}}\right)$ obsc & major isomer & \\
\hline & $\mathrm{H}_{\mathrm{g}}$ & $y$ & $2.02\left(\mathrm{H}_{1}\right)$ & $\mathrm{H}_{\mathrm{d}}$ & $\mathrm{y}$ \\
\hline & $\mathrm{H}_{1}$ & $\mathrm{y}$ & & & \\
\hline \multirow[t]{3}{*}{$3.29\left(\mathrm{H}_{\mathrm{e}}\right)$} & $\mathrm{H}_{\mathrm{f}}$ & $y$ & $1.81\left(\mathrm{H}_{\mathrm{m}}\right)$ & $\mathrm{H}_{\mathrm{c}}$ & $\mathrm{y}$ \\
\hline & $\mathrm{H}_{\mathrm{a}}$ & $y$ & & & \\
\hline & $\mathrm{H}_{\mathrm{c}}$ & $y$ & & & \\
\hline \multirow[t]{2}{*}{$3.73\left(\mathrm{H}_{\mathrm{f}}\right)$} & $\mathrm{H}_{\mathrm{e}}$ & $\mathrm{y}$ & & & \\
\hline & $\mathrm{H}_{\mathrm{i}}$ & $y$ & & & \\
\hline \multirow[t]{2}{*}{$3.35\left(\mathrm{H}_{\mathrm{g}}\right)$} & $\mathrm{H}_{\mathrm{h}}$ & $y$ & & & \\
\hline & $\mathrm{H}_{\mathrm{b}}$ & $y$ & & & \\
\hline
\end{tabular}


Table $3^{\mathbf{a}}$ Solvent Effect of Conformer Distrbiution in $\mathbf{3 e .}$

\begin{tabular}{|c|c|c|c|c|}
\hline Entry & Solvent & $\begin{array}{c}\text { Temperature } \\
{ }^{\circ} \mathrm{C} \quad \mathrm{p}\end{array}$ & $\begin{array}{l}\text { e Ratio } \\
\text { partialcone: cone }\end{array}$ & $\begin{array}{l}\text { Dielectric constants } \\
\text { of non-deuterated } \\
\text { solvents }\end{array}$ \\
\hline 1 & toluene-d 8 & 25 & $2.63: 1$ & 2.38 \\
\hline \multirow[t]{3}{*}{2} & $\mathrm{CDCl}_{3}$ & -30 & $3.94: 1$ & 4.81 \\
\hline & & 25 & $3.9: 1$ & \\
\hline & & 50 & $3.4: 1$ & \\
\hline 3 & Acetone- $\mathrm{d}_{6}$ & 25 & $2.7: 1$ & 20.7 \\
\hline \multirow[t]{2}{*}{4} & $\mathrm{DMSO}^{-\mathrm{d}_{6}}$ & 25 & $1.7: 1$ & 46.7 \\
\hline & & $40-90$ & $"$ & \\
\hline
\end{tabular}

a) Ratio determined from the relative integral values of methyl hydrogens in major and minor isomer

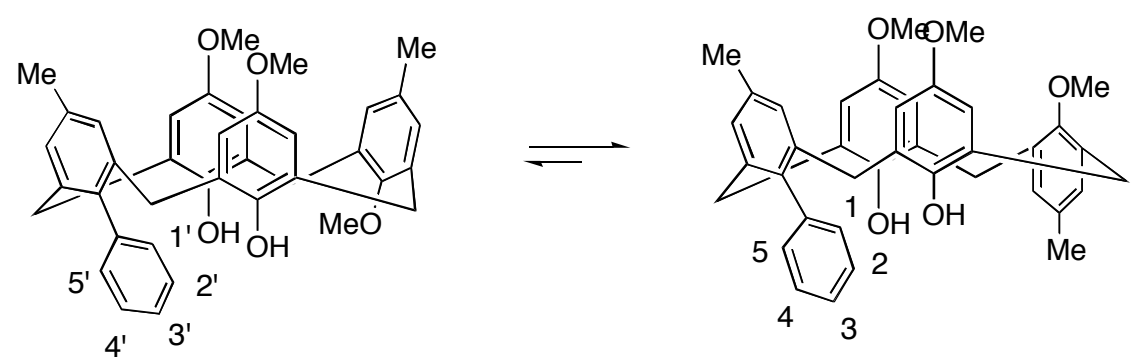

Table 4 Chemical shift of Aromatic hydrogens of $\mathbf{3 e}$ in $\mathrm{DMSO}_{-} \mathrm{d}_{6}$ as solvent

\begin{tabular}{ccc}
\hline Proton & Chemical shift & Multiplicity \\
\hline $\mathrm{H}(1)$ & 4.68 & $\mathrm{~d}$ \\
$\mathrm{H}(2)$ & 5.27 & $\mathrm{t}$ \\
$\mathrm{H}(3)$ & $\mathrm{ND}$ & $\mathrm{t}$ \\
$\mathrm{H}(4)$ & 6.99 & $\mathrm{t}$ \\
$\mathrm{H}(5)$ & $6.74-6.79$ & $\mathrm{~d}$ \\
$\mathrm{H}\left(1^{\prime}\right)$ & 7.73 & $\mathrm{~d}$ \\
$\mathrm{H}\left(2^{\prime}\right)$ & $7.26-7.29$ & $\mathrm{t}$ \\
$\mathrm{H}\left(3^{\prime}\right)$ & $\mathrm{ND}$ & $\mathrm{t}$ \\
$\mathrm{H}\left(4^{\prime}\right)$ & $6.58-6.62$ & $\mathrm{t}$ \\
$\mathrm{H}\left(5^{\prime}\right)$ & $6.74-6.79$ & $\mathrm{~d}$ \\
\hline
\end{tabular}




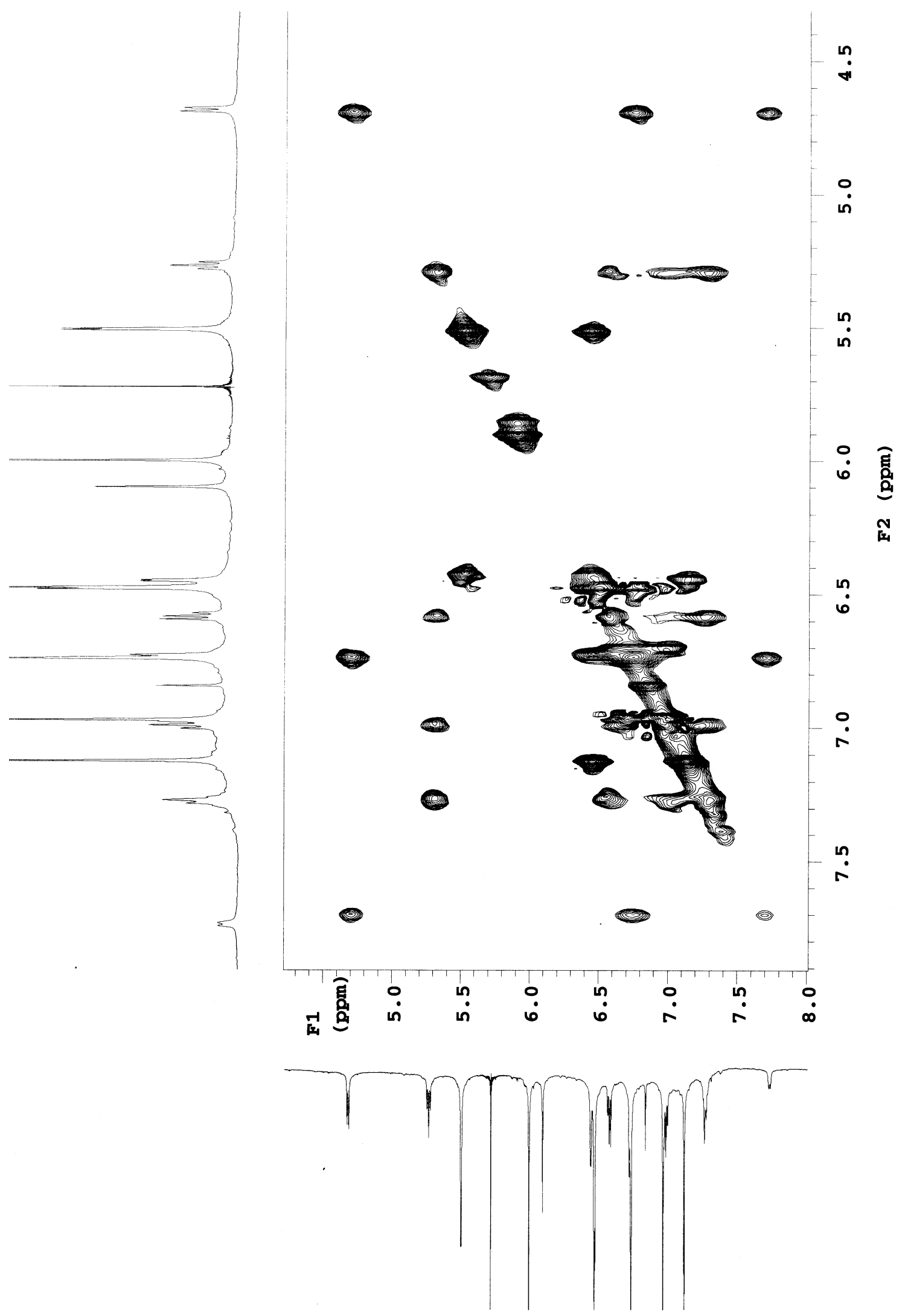

Figure $1600 \mathrm{MHz}{ }^{1} \mathrm{H}$ EXSY spectrum ( $\delta 4.5-8.0 \mathrm{ppm}$ ) of $3 \mathrm{e}$ in DMSO-d $\mathrm{d}_{6}$ at $50^{\circ} \mathrm{C}$. 


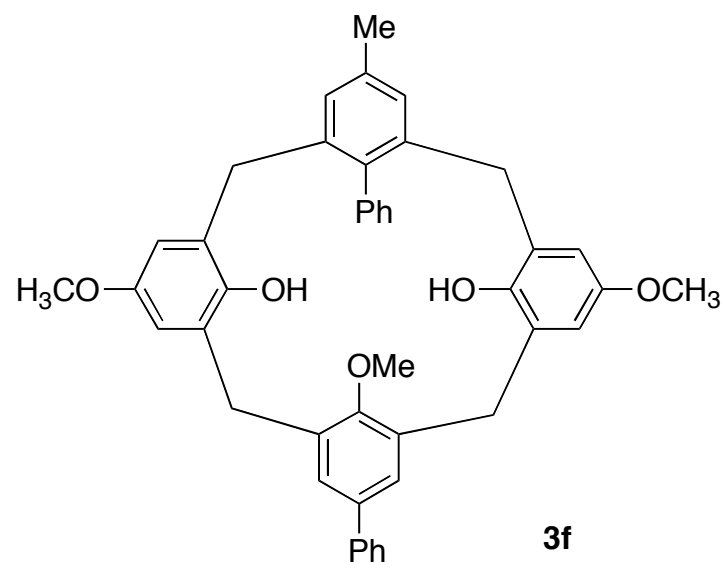

5-methyl-17,28-diphenyl-11,23,26-trimethoxy-25,27-dihydroxycalix(4)arene 3f: The biscarbene complex $1 \mathrm{~d}(0.109 \mathrm{~g}, 0.149 \mathrm{mmol})$ and diyne $2 \mathrm{a}(0.037 \mathrm{~g}, 0.149 \mathrm{mmol})$ were dissolved in $60 \mathrm{~mL}$ of 1,2-dichloroethane and reacted according to the general procedure to give calixarene $\mathbf{3 f}$ as an inseparable 3.3:1 mixture of conformers in $35 \%$ total yield as a white solid after purification by silica gel chromatography ( $25 \%$ ethyl acetate/hexanes). $\mathrm{R}_{\mathrm{f}}=0.45$ (hexanes/ ethyl acetate $=$ 3/1). HPLC analysis showed the presence of single peak at 4.02 min upon gradient elution with mixtures of hexane/iso-propanol varying from $99.5 / 0.5$ to $97 / 3$ over $40 \mathrm{~min}$ at a flow rate of 1 $\mathrm{mL} / \mathrm{min}$ using a silica gel column (R0086100C5). However, the ${ }^{1} \mathrm{H}$ NMR reveals a mixture of two conformers in a $3.3: 1$ ratio. The assignment of the major isomer as a partial cone conformer was made on the basis of the similarity of the proton spectra with that of $3 \mathbf{e}$. The minor conformation was not assigned. The product was further purified by crystallization from hexanes/dichloromethane and the following spectral data was recorded on the mixture of the two conformers. The mass spectrum of $\mathbf{3 f}$ shows a trace peak at $\mathrm{m} / \mathrm{z}=1297(0.13)$. This is attributed to a trace of a calix[8]arene that can not be detected by ${ }^{1} \mathrm{H}$ NMR. A calix[8]arene would not be expected to exists as conformers that could be observed on the ${ }^{1} \mathrm{H}$ NMR time scale. $\mathrm{Mp}=243$ $245{ }^{\circ} \mathrm{C}$. Spectral data for $3 f:{ }^{1} \mathrm{H}$ NMR $\left(\mathrm{CDCl}_{3}, 500 \mathrm{MHz}\right)$ major; $\delta 2.43(\mathrm{~s}, 3 \mathrm{H}), 3.37(\mathrm{~d}, 2 \mathrm{H}, \mathrm{J}=$ $13.0 \mathrm{~Hz}$ ), 3.46 (s, $6 \mathrm{H}$ ), 3.50 (d, $2 \mathrm{H}, \mathrm{J}=16.0 \mathrm{~Hz}$ ), 3.86 (d, $2 \mathrm{H}, \mathrm{J}=16.0 \mathrm{~Hz}$ ), 3.90 (s, $3 \mathrm{H}), 4.18$ $(\mathrm{d}, 2 \mathrm{H}, \mathrm{J}=13.0 \mathrm{~Hz}), 4.86(\mathrm{~d}, 1 \mathrm{H}, \mathrm{J}=7.5 \mathrm{~Hz}), 5.28\left(\mathrm{~s}, \mathrm{CH}_{2} \mathrm{Cl}_{2}\right), 5.50(\mathrm{t}, 1 \mathrm{H}, \mathrm{J}=7.7 \mathrm{~Hz}), 5.66(\mathrm{~d}$, $2 \mathrm{H}, \mathrm{J}=3.0 \mathrm{~Hz}), 5.77(\mathrm{~s}, 2 \mathrm{H}), 6.53(\mathrm{~d}, 2 \mathrm{H}, \mathrm{J}=3.0 \mathrm{~Hz}), 6.61(\mathrm{t}, 1 \mathrm{H}, \mathrm{J}=7.5 \mathrm{~Hz}), 6.83(\mathrm{~d}, 1 \mathrm{H}, \mathrm{J}=$ $7.5 \mathrm{~Hz}$ ), 7.01 (t, $1 \mathrm{H}, \mathrm{J}=7.5 \mathrm{~Hz}$ ), $7.24(\mathrm{~s}, 2 \mathrm{H}), 7.30$ (s, $2 \mathrm{H}), 7.33(\mathrm{~m}, 1 \mathrm{H}), 7.42(\mathrm{t}, 2 \mathrm{H}, \mathrm{J}=7.5$ $\mathrm{Hz}), 7.48(\mathrm{~d}, 2 \mathrm{H}, \mathrm{J}=7.0 \mathrm{~Hz}$ ), minor; $1.60(\mathrm{~s}, 3 \mathrm{H}), 3.27(\mathrm{~d}, 2 \mathrm{H}, \mathrm{J}=14.0 \mathrm{~Hz}), 3.43(\mathrm{~d}, 2 \mathrm{H}, \mathrm{J}=$ 14.0 Hz), 3.74 (d, $2 \mathrm{H}, \mathrm{J}=14.0 \mathrm{~Hz}$ ), $4.21(\mathrm{~d}, 2 \mathrm{H}, \mathrm{J}=14.5 \mathrm{~Hz}), 5.02(\mathrm{~s}, 2 \mathrm{H}), 6.28$ (s, $2 \mathrm{H}), 6.69$ $(\mathrm{d}, 2 \mathrm{H}, \mathrm{J}=3.0 \mathrm{~Hz}), 6.91(\mathrm{~d}, 1 \mathrm{H}, \mathrm{J}=7.5 \mathrm{~Hz}), 6.96(\mathrm{~s}, 2 \mathrm{H})$. The remaining 11 Ar-H's of the minor conformer overlap with the peak resonances of the major isomer and hence their positions could 
not be precisely assigned. ${ }^{13} \mathrm{C} \mathrm{NMR}\left(\mathrm{CDCl}_{3}, 125 \mathrm{MHz}\right) \delta 20.54,21.19,31.33,32.06,36.10$, $39.98,55.33$, 55.68, 63.18, 63.31, 113.11, 113.67, 113.92, 114.64, 125.35, 126.15, 126.65, $126.91,126.95,127.03,127.19,127.66,127.74,127.79,127.91,128.47,128.79,129.53,129.74$, $130.11,130.72,131.28,132.09,132.84,133.52,133.60,134.22,136.48,136.80,137.71,138.38$, 138.50, 138.53, 139.01, 139.26, 139.57, 140.56, 140.64, 146.61, 147.01, 152.47, 152.54, 153.52, 153.70; IR $\left(\mathrm{CH}_{2} \mathrm{Cl}_{2}\right) 3493 \mathrm{br}, 3055 \mathrm{~s}, 2988 \mathrm{~m}, 1653 \mathrm{~m}, 1609 \mathrm{~m}, 1481 \mathrm{~m}, 1421 \mathrm{~m}, 1265 \mathrm{~s}, 1147 \mathrm{w} \mathrm{cm}^{-1}$; mass spectrium (FAB MS in 4-nitrobenzyl alcohol) $\mathrm{m} / \mathrm{z}$ (\% rel intensity) $648 \mathrm{M}^{+}(100), 307$ (30), 154 (100), 136 (60), 107 (20), 77 (20), calcd for $\mathrm{C}_{44} \mathrm{H}_{40} \mathrm{O}_{5} \mathrm{~m} / \mathrm{z}$ 648.2876, measd 648.2874.

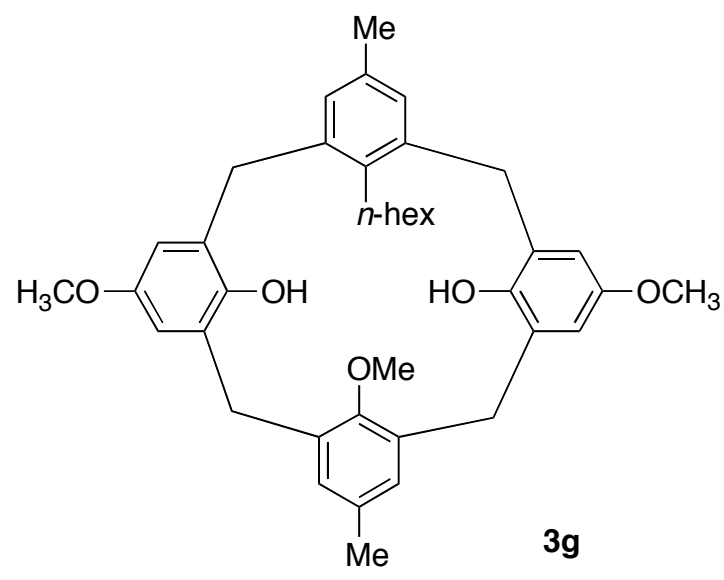

5,17-dimethyl-11,23,26-trimethoxy-25,27-dihydroxy-28-hexylcalix(4)arene $3 g$ : A solution of the bis-carbene complex $1 \mathrm{c}(0.207 \mathrm{~g}, 0.31 \mathrm{mmol})$ and diyne $2 \mathrm{~b}(0.077 \mathrm{~g}, 0.307 \mathrm{mmol})$ in $122 \mathrm{~mL}$ of 1,2-dichloroethane was subjected was allowed to react according to the general procedure described above. The product of this reaction was purified by silica gel chromatography (hexanes/ethyl acetate $=85 / 15$ ) to give calixarene $\mathbf{3 g}$ in $22 \%$ yield as an offwhite solid and as a inseparable 7.9:1 mixture of conformers. $R_{f}=0.31$ (15\% EtOAc / hexanes). $\mathrm{Mp}=172-174{ }^{\circ} \mathrm{C}$. HPLC analysis showed the presence of single peak at $6.02 \mathrm{~min}$ under gradient elution with a mixture of hexane/iso-propanol starting at $99.5 / 0.5$ and decreasing to $97 / 3$ over 40 min at a flow rate of $1 \mathrm{~mL} / \mathrm{min}$ on a silica gel column (R0086100C5). The ${ }^{1} \mathrm{H}$ NMR indicates the presence of a 7.9:1 mixture of conformers as measured by integration of the peaks at $\delta=1.91$ and 2.16. The major isomer was assigned as the cone conformer on the basis of NOE and NOESY experiments (see below). The minor isomer was not assigned. NOESY experiment on 3g: $\mathrm{Tm}=0.5 \mathrm{sec}, \mathrm{nt}=256, \mathrm{ni}=64$, linear prediction along $\mathrm{F} 1$ dimension.

The following spectral data was obtained on a mixture of the conformers. The mass spectrum of $\mathbf{3 g}$ shows a trace peak at $\mathrm{m} / \mathrm{z}=1189(0.20)$. This is attributed to a trace of a calix[8]arene that can not be detected by ${ }^{1} \mathrm{H}$ NMR. A calix[8]arene would not be expected to exists as conformers that could be observed on the ${ }^{1} \mathrm{H}$ NMR time scale. Spectral data for $\mathbf{3 g}$ : ${ }^{1} \mathrm{H}$ 
$\operatorname{NMR}\left(\mathrm{CDCl}_{3}, 500 \mathrm{MHz}\right)$ major; $\delta 0.74(\mathrm{t}, 3 \mathrm{H}, \mathrm{J}=7.2 \mathrm{~Hz}), 1.31(\mathrm{~m}, 4 \mathrm{H}), 1.39(\mathrm{~m}, 4 \mathrm{H}), 1.91(\mathrm{~s}, 3$ H), 2.04 (s, $3 \mathrm{H}$ ), 3.29 (t, $2 \mathrm{H}, \mathrm{J}=7.3 \mathrm{~Hz}$ ), 3.39 (d, $2 \mathrm{H}, \mathrm{J}=14.0 \mathrm{~Hz}$ ), 3.40 (d, $2 \mathrm{H}, \mathrm{J}=14.0 \mathrm{~Hz}$ ), $3.79(\mathrm{~s}, 6 \mathrm{H}), 3.88(\mathrm{~s}, 3 \mathrm{H}), 4.00(\mathrm{~d}, 2 \mathrm{H}, \mathrm{J}=14.0 \mathrm{~Hz}), 4.21(\mathrm{~d}, 2 \mathrm{H}, \mathrm{J}=13.5 \mathrm{~Hz}), 5.78(\mathrm{~s}, 2 \mathrm{H})$, $6.54(\mathrm{~s}, 2 \mathrm{H}), 6.63(\mathrm{~d}, 2 \mathrm{H}, \mathrm{J}=3.0 \mathrm{~Hz}), 6.69(\mathrm{~s}, 2 \mathrm{H}), 6.71(\mathrm{~d}, 2 \mathrm{H}, \mathrm{J}=2.5 \mathrm{~Hz})$, minor; $0.88(\mathrm{t}, 3 \mathrm{H}$, $\mathrm{J}=7.2 \mathrm{~Hz}$ ), 1.24 (bs, $4 \mathrm{H}$ ), 1.48 (bs, $4 \mathrm{H}$ ), 2.16 (s, $3 \mathrm{H}), 2.35$ (s, $3 \mathrm{H}), 3.23$ (d, $2 \mathrm{H}, \mathrm{J}=12.5 \mathrm{~Hz}$ ), 3.69 (s, $6 \mathrm{H}), 3.77$ (s, 3 H), $3.91(\mathrm{~d}, 2 \mathrm{H}, \mathrm{J}=12.5 \mathrm{~Hz}), 3.99(\mathrm{~d}, 2 \mathrm{H}, \mathrm{J}=12.5 \mathrm{~Hz}), 4.08$ (d, $2 \mathrm{H}, \mathrm{J}=$ $12.5 \mathrm{~Hz}$ ), 5.75 (s, $2 \mathrm{H}$ ), 6.52 (d, $2 \mathrm{H}, \mathrm{J}=3.0 \mathrm{~Hz}$ ), 6.58 (d, $2 \mathrm{H}, \mathrm{J}=3.5 \mathrm{~Hz}$ ), 6.87 (s, $2 \mathrm{H}$ ), 7.14 (s,

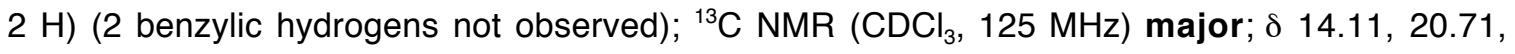
20.72 , 22.72, 28.48, 29.56, 31.96, 32.34, 32.87, 35.68, 55.77, 63.38, 113.35, 114.4, 128.37, $128.98,129.95,131.58,132.16,134.01,134.99$, 137.27, 137.73, 147.10, 150.72, 152.58; IR $\left(\mathrm{CH}_{2} \mathrm{Cl}_{2}\right) 3437 \mathrm{br}, 2924 \mathrm{~s}, 2855 \mathrm{~m}, 1605 \mathrm{~m}, 1482 \mathrm{~s}, 1225 \mathrm{~s}, 1145 \mathrm{~m}, 1053 \mathrm{~s} \mathrm{~cm}^{-1}$; mass spectrum $\mathrm{m} / \mathrm{z}$ (\% rel intensity) $594 \mathrm{M}^{+}$(100), 307 (10), 154 (35), 136 (20), calcd for calculated for $\mathrm{C}_{39} \mathrm{H}_{46} \mathrm{O}_{5} \mathrm{~m} / \mathrm{z}$ 594.3345, measd 594.3344. 


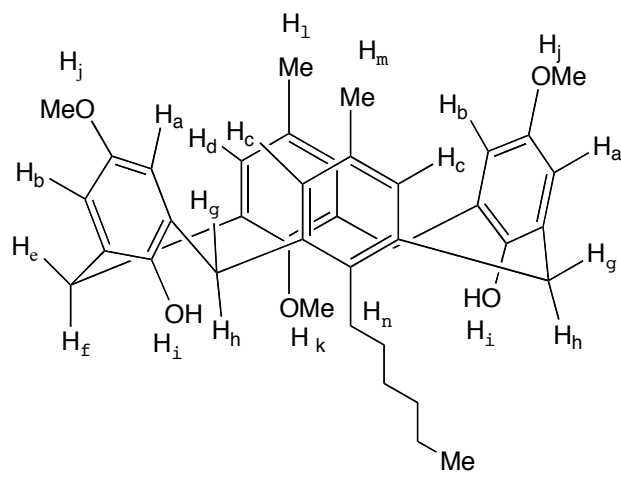

3g Major Isomer (Cone Conformer)

\begin{tabular}{|cr|}
$\begin{array}{c}\text { Chemical Shifts For } \mathbf{H}_{\mathrm{a}}-\mathbf{H}_{\mathbf{n}} \text { Major Isomer of } \mathbf{3 g} \\
\delta \mathrm{ppm}\end{array}$ & \\
\hline $\mathrm{H}_{\mathrm{a}}=6.63(\mathrm{~d})$ & $\mathrm{H}_{\mathrm{j}}=3.80(\mathrm{~s})$ \\
$\mathrm{H}_{\mathrm{b}}=6.71(\mathrm{~d})$ & $\mathrm{H}_{\mathrm{k}}=3.88(\mathrm{~s})$ \\
$\mathrm{H}_{\mathrm{c}}=6.69(\mathrm{~s})$ & $\mathrm{H}_{\mathrm{l}}=1.92(\mathrm{~s})$ \\
$\mathrm{H}_{\mathrm{d}}=6.54(\mathrm{~s})$ & $\mathrm{H}_{\mathrm{m}}=2.04(\mathrm{~s})$ \\
$\mathrm{H}_{\mathrm{e}}=3.39(\mathrm{~d})$ & \\
$\mathrm{H}_{\mathrm{f}}=4.00(\mathrm{~d})$ & \\
$\mathrm{H}_{\mathrm{g}}=3.40(\mathrm{~d})$ & \\
$\mathrm{H}_{\mathrm{h}}=4.21(\mathrm{~d})$ & \\
$\mathrm{H}_{\mathrm{i}}=5.78(\mathrm{~s})$ & \\
\hline
\end{tabular}

Table 5 Results of Homonuclear ${ }^{1} \mathrm{H}-{ }^{1} \mathrm{H}$ NOE Experiments on the major conformer of $\mathbf{3 g}$.

\begin{tabular}{|c|c|c|c|c|c|}
\hline Nuclei irradiated & Adjacent $\mathrm{H}$ & Enhancement & Nuclei irradiated & Adjacent $\mathrm{H}$ & enhancement \\
\hline \multirow[t]{2}{*}{$6.63\left(\mathrm{H}_{\mathrm{a}}\right)$} & $\mathrm{H}_{\mathrm{g}}$ & y & \multirow[t]{2}{*}{$3.40\left(\mathrm{H}_{\mathrm{g}}\right)$ or $\mathrm{H}_{\mathrm{e}}$} & $\mathrm{H}_{\mathrm{h}}$ & y \\
\hline & $\mathrm{H}_{j}$ & $y$ & & $\mathrm{H}_{\mathrm{c}}$ & $\mathrm{y}$ \\
\hline \multirow[b]{2}{*}{$6.71\left(\mathrm{H}_{\mathrm{b}}\right)$} & $W_{j}$ & $y$ & \multirow{3}{*}{$4.21\left(\mathrm{H}_{\mathrm{h}}\right)$} & $\mathrm{H}_{\mathrm{a}}$ & y \\
\hline & $\mathrm{H}_{\mathrm{j}}$ & $\mathrm{y}$ & & $\mathrm{H}_{\mathrm{g}}$ & y \\
\hline \multirow{3}{*}{$6.69\left(\mathrm{H}_{\mathrm{c}}\right)$} & $\mathrm{H}_{\mathrm{e}}$ (or) $\mathrm{H}_{\mathrm{g}}$ & $\mathrm{n}$ & & $\mathrm{H}_{\mathrm{n}}$ & y \\
\hline & $\mathrm{H}_{\mathrm{g}}$ (or) $\mathrm{H}_{\mathrm{e}}$ & $\mathrm{y}$ & & $\mathrm{H}_{\mathrm{k}}$ & $n$ \\
\hline & $\mathrm{H}_{\mathrm{m}}$ & y & $5.78\left(\mathrm{H}_{\mathrm{i}}\right)$ & \multicolumn{2}{|c|}{ NO NOE } \\
\hline \multirow[t]{2}{*}{$6.54\left(\mathrm{H}_{\mathrm{d}}\right)$} & $\mathrm{H}_{\mathrm{l}}$ & y & \multirow[t]{2}{*}{$3.80\left(\mathrm{H}_{j}\right)$} & $\mathrm{H}_{\mathrm{a}}$ & y \\
\hline & $\mathrm{H}_{\mathrm{e}}$ & $\mathrm{n}$ & & $\mathrm{H}_{\mathrm{b}}$ & y \\
\hline $3.39\left(\mathrm{H}_{\mathrm{e}}\right)$ & $\mathrm{H}_{\mathrm{f}}$ & Y & $3.88\left(\mathrm{H}_{\mathrm{k}}\right)$ & $\mathrm{H}_{\mathrm{f}}$ & $y$ \\
\hline \multirow[t]{2}{*}{ or $\left(\mathrm{H}_{\mathrm{g}}\right)$} & $\mathrm{H}_{\mathrm{b}}$ & y & \multirow[t]{2}{*}{$1.91\left(\mathrm{H}_{l}\right)$} & \multirow[t]{2}{*}{$\mathrm{H}_{\mathrm{d}}$} & \multirow[t]{2}{*}{ y } \\
\hline & $\mathrm{H}_{\mathrm{d}}$ & y & & & \\
\hline \multirow{3}{*}{$4.00\left(\mathrm{H}_{\mathrm{f}}\right)$} & & $y$ & $2.04\left(\mathrm{H}_{\mathrm{m}}\right)$ & $\mathrm{Hc}$ & y \\
\hline & $\mathrm{H}_{\mathrm{e}}$ & y & \multirow[t]{2}{*}{$3.29\left(H_{n}\right)$} & $\mathrm{H}_{\mathrm{h}}$ & $y$ \\
\hline & $\mathrm{H}_{\mathrm{k}}$ & $\mathrm{y}$ & & $\mathrm{CH}_{2}$ & y \\
\hline
\end{tabular}




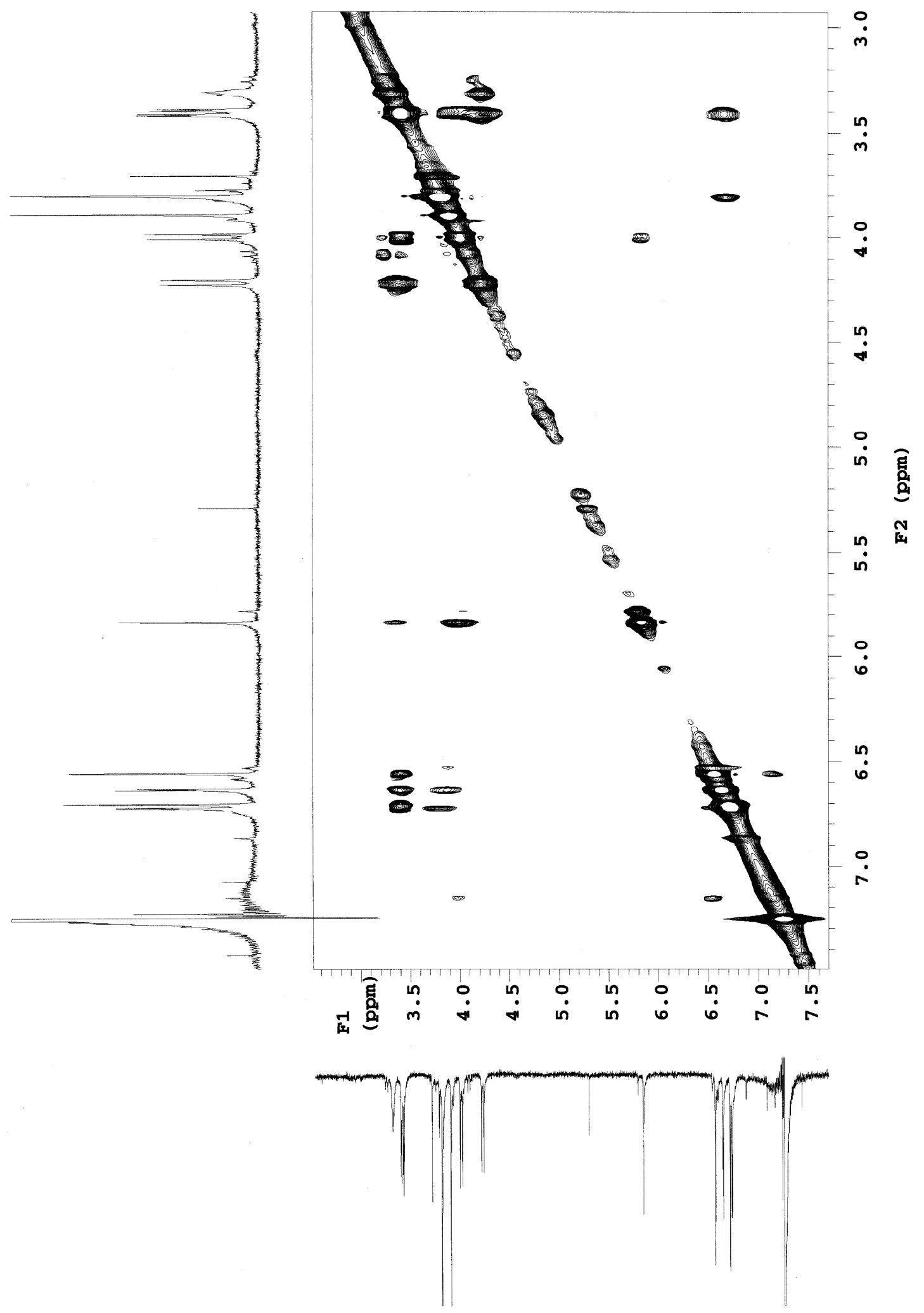

Figure 2. Expanded region of the $600 \mathrm{MHz}$ NOESY spectrum of $\mathbf{3 g}$ in $\mathrm{CDCl}_{3}$ at $25^{\circ} \mathrm{C}$. 


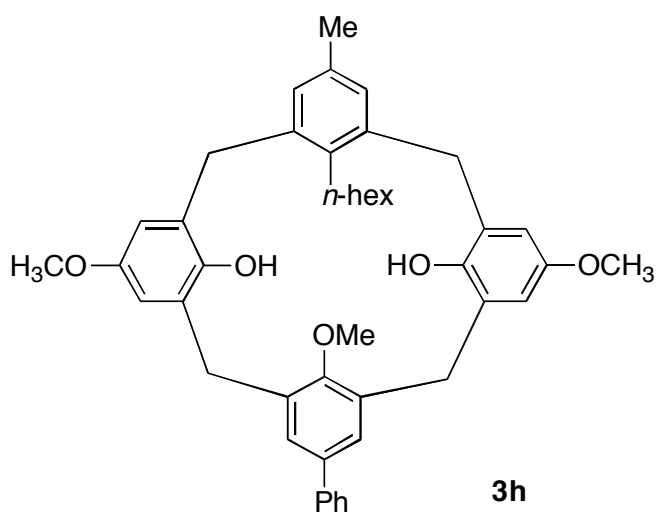

5-phenyl-17-methyl-11,23,26-trimethoxy-25,27-dihydrox-28-hexylcalix(4)arene $3 \mathrm{~h}:$ The reaction of the bis-carbene complex $1 \mathbf{b}(0.103 \mathrm{~g}, 0.142 \mathrm{mmol})$ and diyne $2 \mathrm{~d}(0.037 \mathrm{~g}, 0.142$ $\mathrm{mmol}$ ) in $56 \mathrm{~mL}$ of 1,2-dichloroethane was carried according to the general procedure. The calixarene $3 \mathrm{~h}$ was obtained as an inseparable 7.9:1 mixture of conformers in $35 \%$ yield as an offwhite solid after purification by silica gel chromatography ( $25 \%$ EtOAc / hexanes). $R_{f}=0.38$ (25 $\%$ EtOAc / hexanes). The resulting product was further purified by crystallization from dichlormethane/hexanes $\left(\mathrm{mp}=170-172^{\circ} \mathrm{C}\right)$. HPLC analysis showed the presence of single peak at $4.08 \mathrm{~min}$ with gradient elution with hexane/iso-propanol from $99.5 / 0.5$ to $97 / 3$ over $40 \mathrm{~min}$ at a flow rate of $1 \mathrm{~mL} / \mathrm{min}$ on a silica gel column (R0086100C5). The structure of neither conformer was assigned. The following spectral data was taken on the mixture of conformers of $\mathbf{3 h}:{ }^{1} \mathrm{H}$ NMR $\left(\mathrm{CDCl}_{3}, 500 \mathrm{MHz}\right)$ major; $\delta 0.95(\mathrm{t}, 3 \mathrm{H}, \mathrm{J}=7.2 \mathrm{~Hz}), 1.38(\mathrm{~m}, 4 \mathrm{H}), 1.46(\mathrm{~m}, 4 \mathrm{H}), 1.89(\mathrm{~s}, 3$ H), $3.35(t, 2 \mathrm{H}, \mathrm{J}=7.5 \mathrm{~Hz}$ ), $3.49(\mathrm{~d}, 2 \mathrm{H}, \mathrm{J}=14.0 \mathrm{~Hz}), 3.56(\mathrm{~d}, 2 \mathrm{H}, \mathrm{J}=14.0 \mathrm{~Hz}), 3.87(\mathrm{~s}, 6 \mathrm{H})$, $4.00(\mathrm{~s}, 3 \mathrm{H}), 4.18(\mathrm{~d}, 2 \mathrm{H}, \mathrm{J}=14.0 \mathrm{~Hz}), 4.28(\mathrm{~d}, 2 \mathrm{H}, \mathrm{J}=14.0 \mathrm{~Hz}), 5.63(\mathrm{~s}, 2 \mathrm{H}), 6.58(\mathrm{~s}, 2 \mathrm{H})$, $6.74(\mathrm{~d}, 2 \mathrm{H}, \mathrm{J}=2.5 \mathrm{~Hz}), 6.79(\mathrm{~d}, 2 \mathrm{H}, \mathrm{J}=3 \mathrm{~Hz}), 7.12(\mathrm{~s}, 2 \mathrm{H}), 7.30-7.38(\mathrm{~m}, 5 \mathrm{H})$; ${ }^{13} \mathrm{C}$ NMR $\left(\mathrm{CDCl}_{3}, 125 \mathrm{MHz}\right)$ major + minor; $\delta 13.75,14.11,20.65,20.92,22.48,22.72,28.44,28.66$, 29.38, 29.53, 29.82, 31.27, 31.61, 31.91, 32.38, 32.76, 35.59, 40.42, 55.51, 55.76, 63.01, 63.39, 113.39, 113.83, 114.44, 126.94, 127.07, 127.63, 127.99, 128.54, 128.57, 128.61, 128.87, 128.98, 130.68, 131.35, 131.60, 132.88, 134.29, 134.34, 136.92, 137.72, 138.29, 139.41, 140.41, 147.07, 152.64, 152.75, 152.82 (9 carbons not located); IR $\left(\mathrm{CH}_{2} \mathrm{Cl}_{2}\right) 3485 \mathrm{br}, 2926 \mathrm{~s}, 2855 \mathrm{~s}, 2836 \mathrm{~s}$, 1605s, 1481m, 1236s, 1146s, 1055s cm-1. Anal Calcd for $\mathrm{C}_{44} \mathrm{H}_{48} \mathrm{O}_{5}: \mathrm{C}, 80.46 ; \mathrm{H}, 7.37$. Found: $\mathrm{C}$, $80.50 ; \mathrm{H}, 7.32$. 


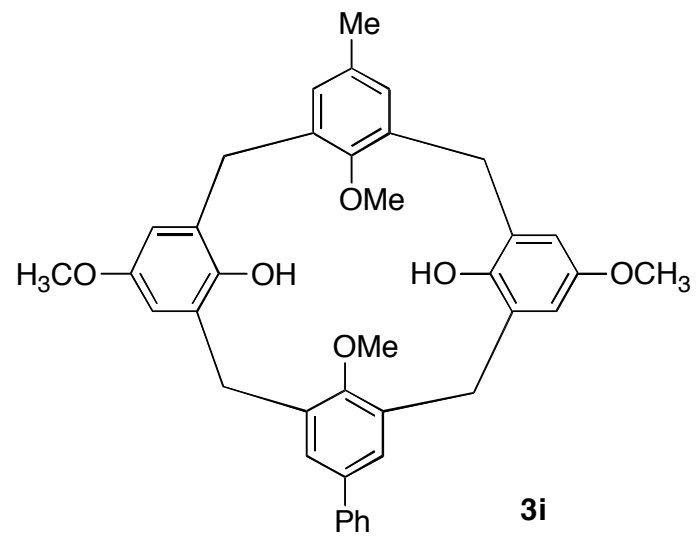

5-phenyl-17-methyl-11,23,26,28-tetramethoxy-25,27-dihydroxycalix(4)arene 3i: The reaction of the bis-carbene complex $1 \mathbf{d}(0.105 \mathrm{~g}, 0.143 \mathrm{mmol})$ and diyne $2 \mathrm{c}(0.029 \mathrm{~g}, 0.145$ $\mathrm{mmol}$ ) was performed as described in the general procedure to give, after purification by silica gel chromatography (25\% EtOAc / hexanes), the calixarene $\mathbf{3 i}$ as a single conformer in $40 \%$ yield. The conformation of the calixarene was assigned as the cone conformer. The resultant product was further purified by crystallization from hexanes/dichloromethane to afford shiny white crystals of 3i. $\mathrm{Mp}=236-239^{\circ} \mathrm{C}$. $\mathrm{R}_{\mathrm{f}}=0.37$ (hexanes $/$ ethyl acetate $=3 / 1$ ). Spectral data for $3 \mathbf{i}:{ }^{1} \mathrm{H}$ NMR $\left(\mathrm{CDCl}_{3}, 500 \mathrm{MHz}\right) \delta 2.02(\mathrm{~s}, 3 \mathrm{H}), 3.31(\mathrm{~d}, 2 \mathrm{H}, \mathrm{J}=13.0 \mathrm{~Hz}$ ), $3.41(\mathrm{~d}, 2 \mathrm{H}, \mathrm{J}=13 \mathrm{~Hz}), 3.76$ (s, 6 H), $3.97(\mathrm{~s}, 3 \mathrm{H}), 4.02(\mathrm{~s}, 3 \mathrm{H}), 4.29(\mathrm{~d}, 2 \mathrm{H}, \mathrm{J}=13.0 \mathrm{~Hz}), 4.39$ (d, $2 \mathrm{H}, \mathrm{J}=13.0 \mathrm{~Hz}), 6.64(\mathrm{~d}, 2 \mathrm{H}$, $\mathrm{J}=3.0 \mathrm{~Hz}$ ), $6.68(\mathrm{~d}, 2 \mathrm{H}, \mathrm{J}=3.0 \mathrm{~Hz}), 6.71(\mathrm{~s}, 2 \mathrm{H}), 7.13(\mathrm{~s}, 2 \mathrm{H}), 7.23(\mathrm{~m}, 1 \mathrm{H}), 7.31(\mathrm{~s}, 2 \mathrm{H}), 7.32$ (s, $2 \mathrm{H}), 7.42$ (s, $2 \mathrm{H}) ;{ }^{13} \mathrm{C}$ NMR $\left(\mathrm{CDCl}_{3}, 125 \mathrm{MHz}\right) \delta 20.82,31.54,31.72$, 55.85, 55.89, 63.52, 113.85, 113.93, 126.89, 127.80, 128.54, 129.08, 129.21, 129.74, 132.64, 133.49, 134.41, 137.72, 140.62, 146.96, 151.35, 152.39, 153.39 (1 aryl carbon not located); $\operatorname{IR}\left(\mathrm{CH}_{2} \mathrm{Cl}_{2}\right) 3354 \mathrm{br}, 2930 \mathrm{~m}$, 2829w, 1599w, 1483s, 1435m, 1244m, 1142w, 1053w cm ${ }^{-1}$; mass spectrum El m/z (\% rel intensity) $602 \mathrm{M}^{+}$(100), 571 (5), 539 (8), 301 (10), calcd for $\mathrm{C}_{39} \mathrm{H}_{38} \mathrm{O}_{6} \mathrm{~m} / \mathrm{z}$ 602.2668, measd 602.2668.

\section{References:}

1. a) Watson, S. C; Eastham, J.F. J.Organometal.Chem.1967, 9, 165

b) Paquette, L.A.; Lin, Ho-Shen. Syn.Comm.1994, 24, 2503

2. Cram, D.J.; Koenig, K.E.; Lein, G.M.; Stuckler, P.; Kaneda, T. J.Am.Chem.Soc.1979, 3553.

3. Weber, E.; Trepte, J.; Gloe, K.; Piel, M.; Czugler, M.; Kravtsov, V.C.; Simonov, Y.A.; Lipkowski, J.; Ganin, E.V. J.Chem.Soc.Perkin.Trans.2, 1996, 2359.

4. Huang, W.; Gou, S.; Meng, Q., Syn. Comm., 2000, 30, 1555.

5. Frantz, D.E.; Weaver, D.G.; Carey, J.P.; Kress, M.H.; Dolling, U.H. Org.Lett. 2002, 4, 4717.

6. Müller, W.; Kipfer, P.; Lowe, D.A.; Urwyler, S. Helv.Chim.Acta.1995, 78, 2026.

7. Suzuki, M.; Kimura, Y.; Terashima, S., Chem. Pharm. Bull, 1986, 34, 1531.

8. Lipshutz, B. H.; Keil, R.; Ellsworth, E. L., Tetrahedron Lett., 1990, 50, 7257.

9. Van Gelder, J. M.; Brenn, J.; Thondorf, I.; Biali, S. E., J. Org. Chem., 1997, 62, 3511. 
${ }^{1} \mathrm{H}$ NMR Spectra of Calixarenes $\mathbf{3 a} \mathbf{-} \mathbf{3 i}$. 

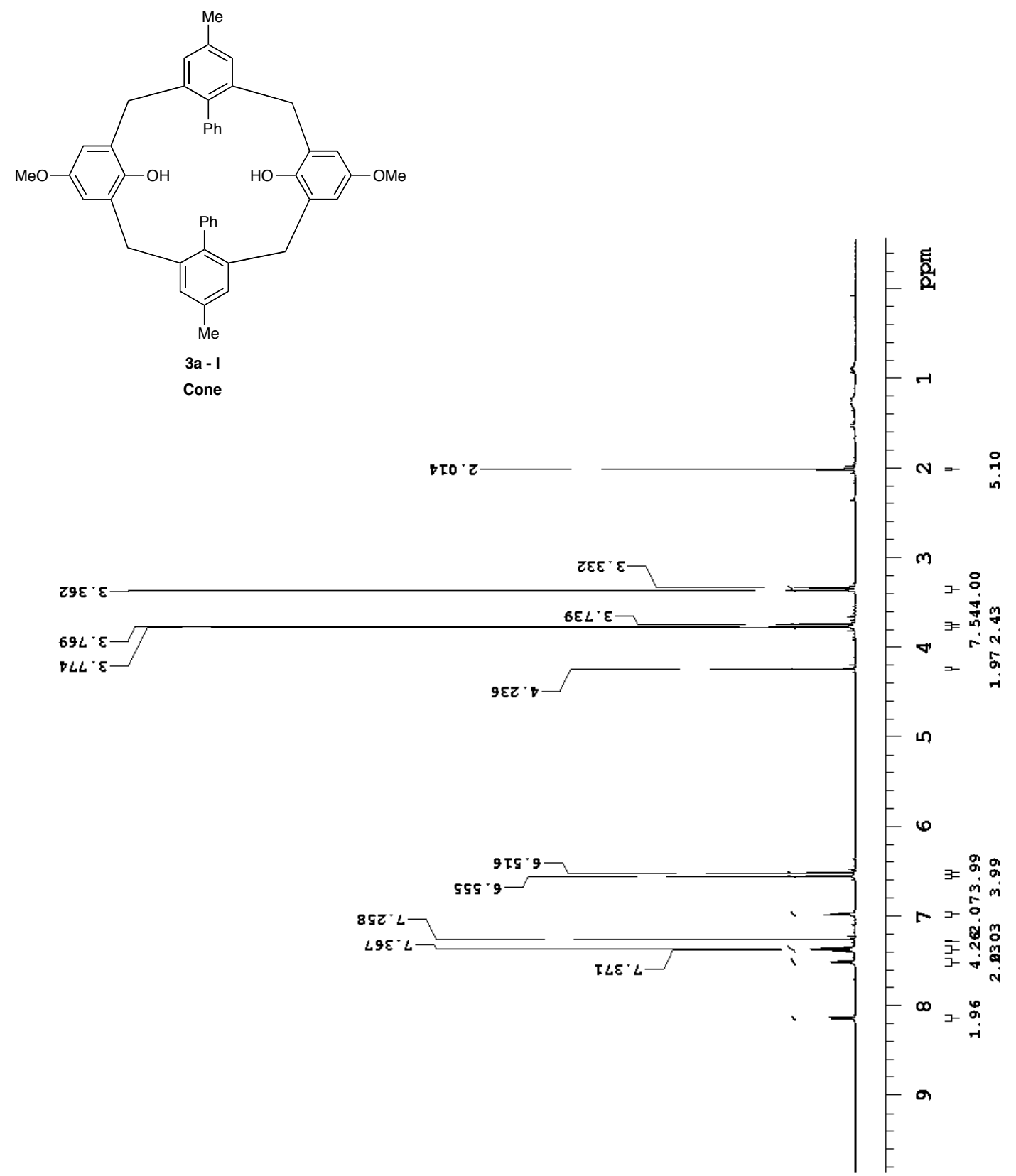

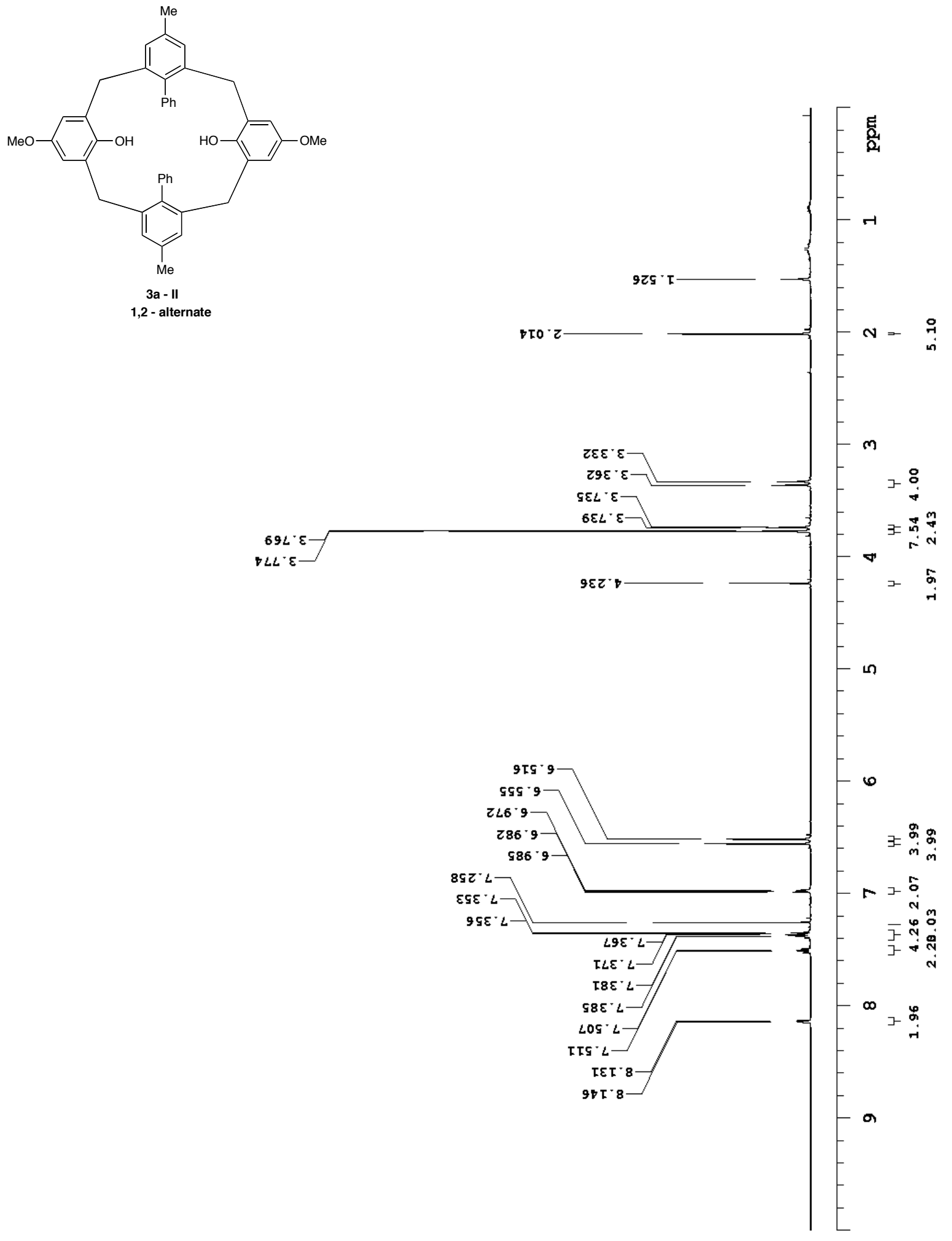

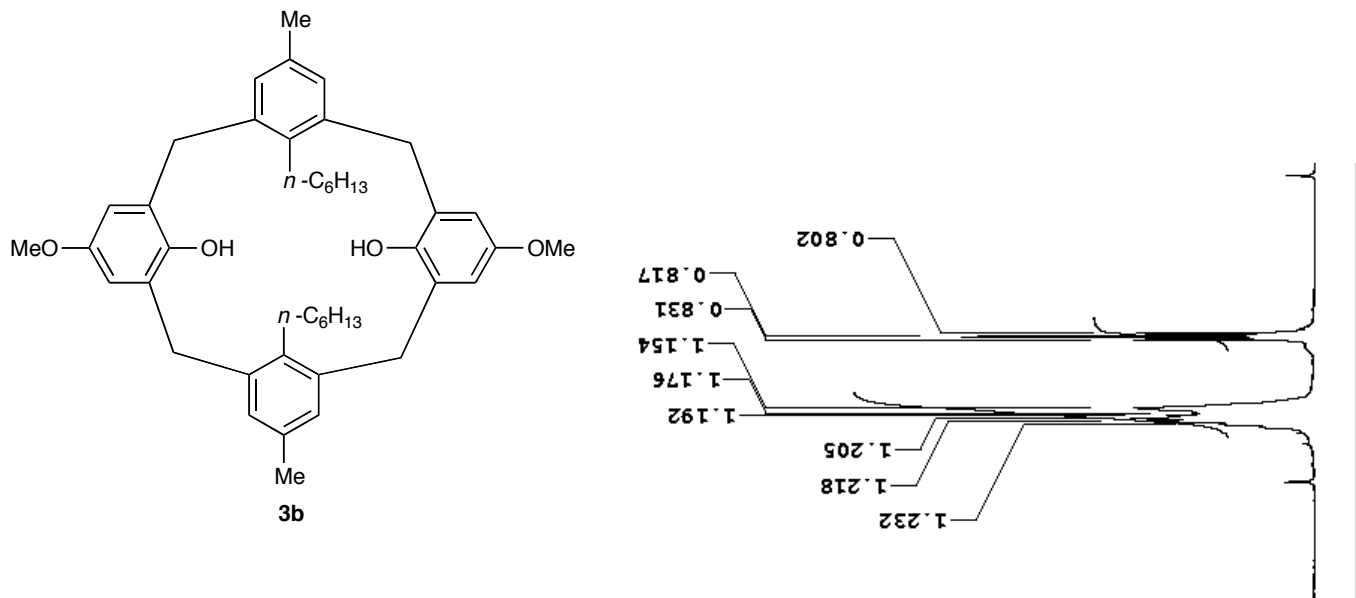

总

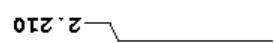
tos $z$

$9 \mathrm{Ts} z$

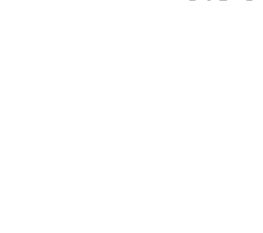

Oโร $\varepsilon$

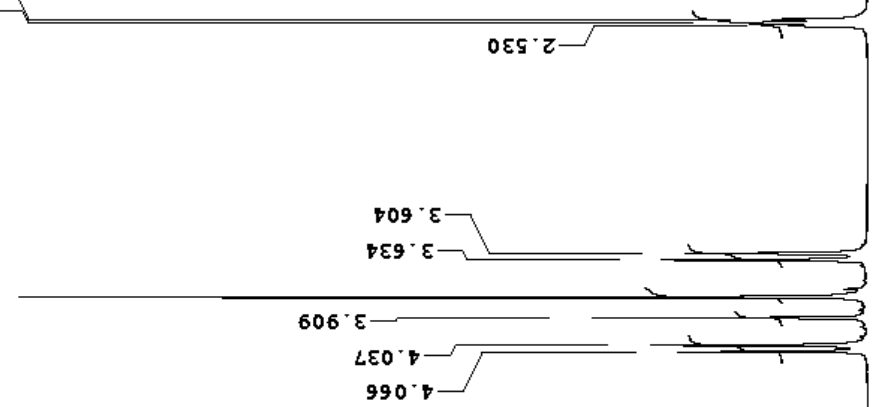

N

ᄀ

了

$m$

了 ถั

J它

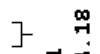

굴

$\nabla \sqrt{-7}$

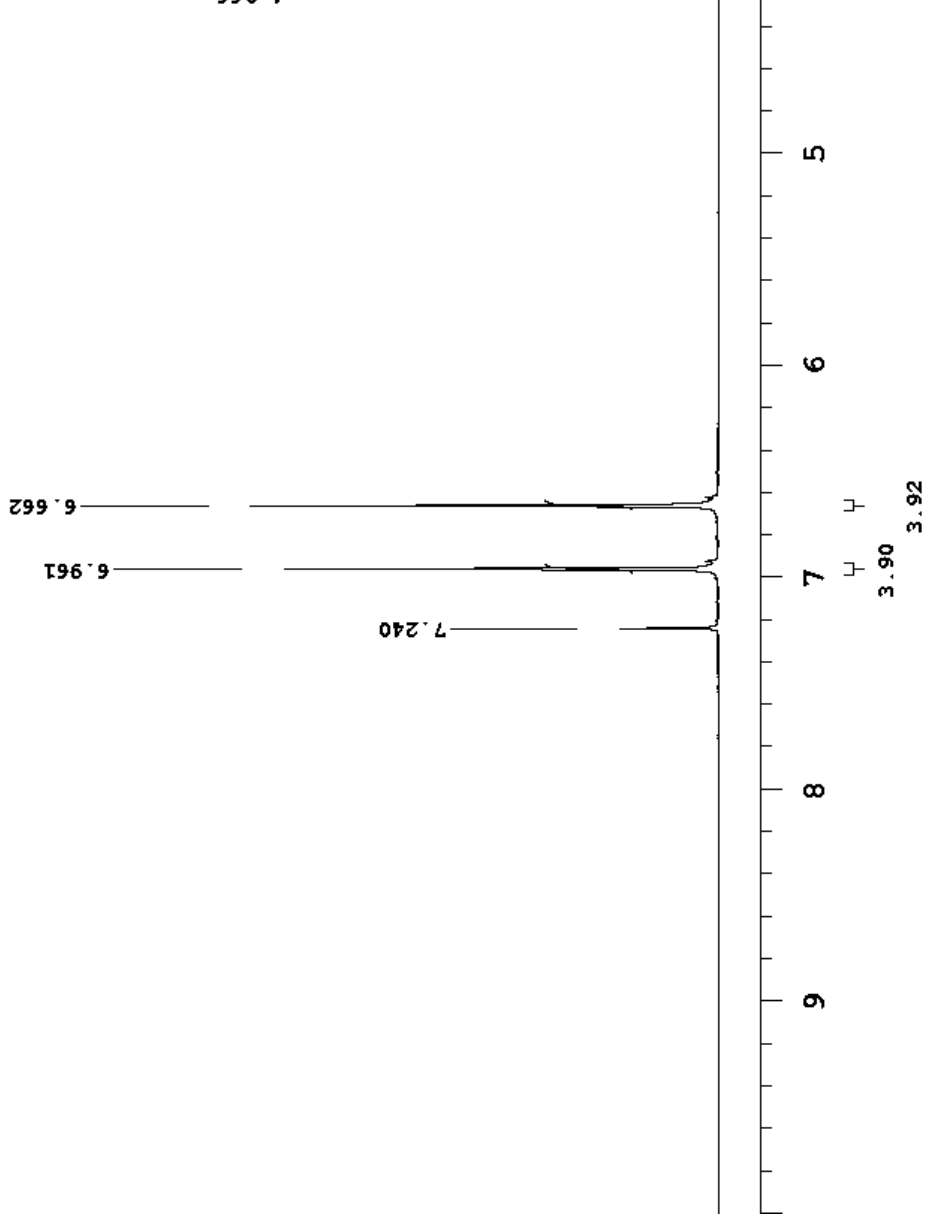




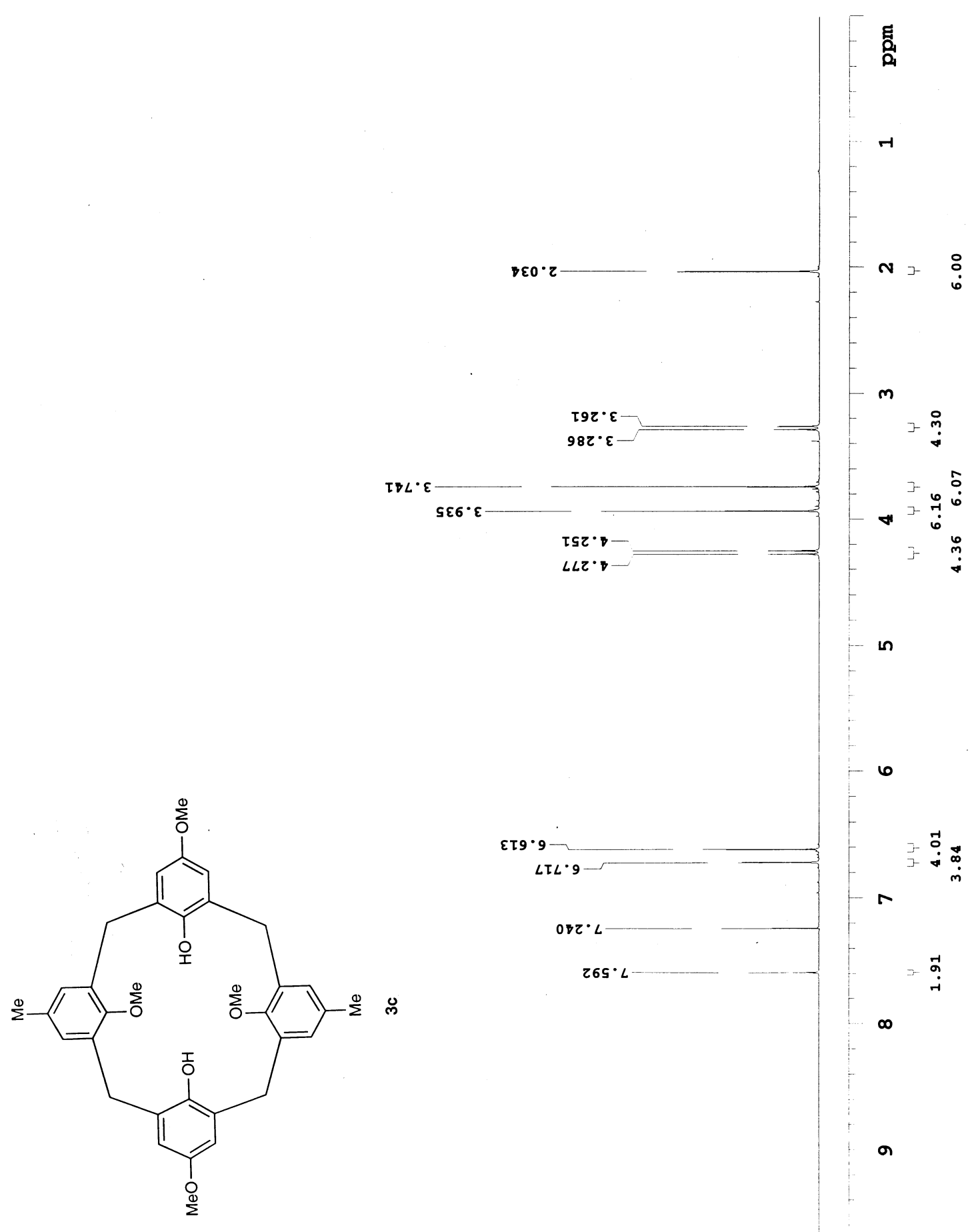



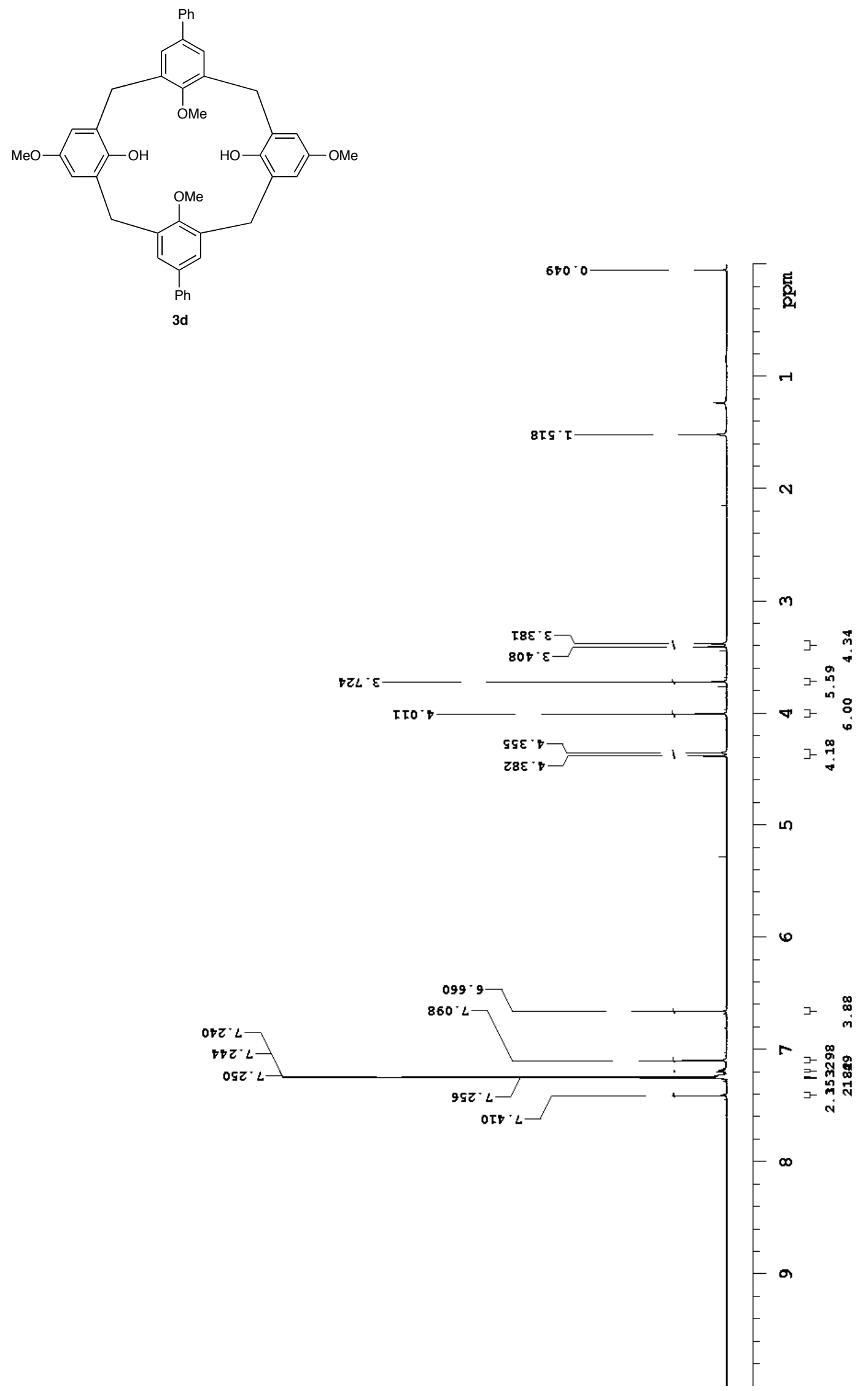

S-36 


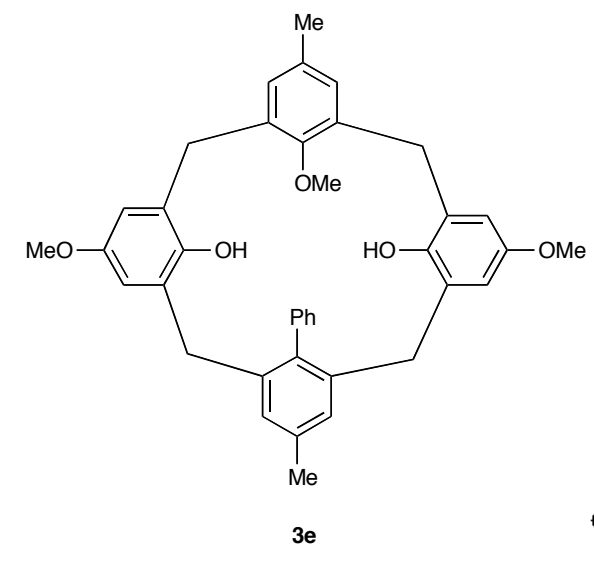

| F

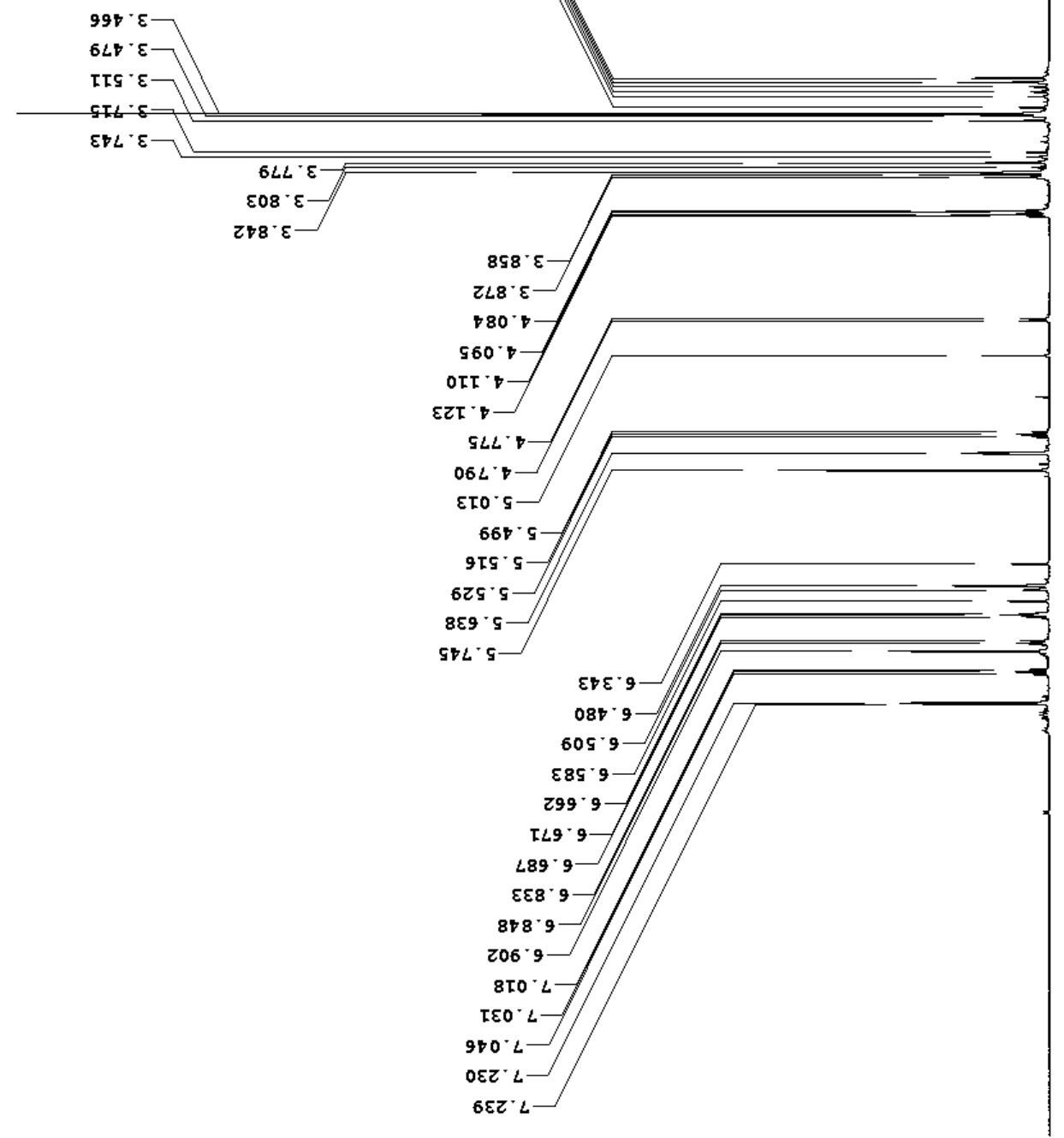

$m$ 

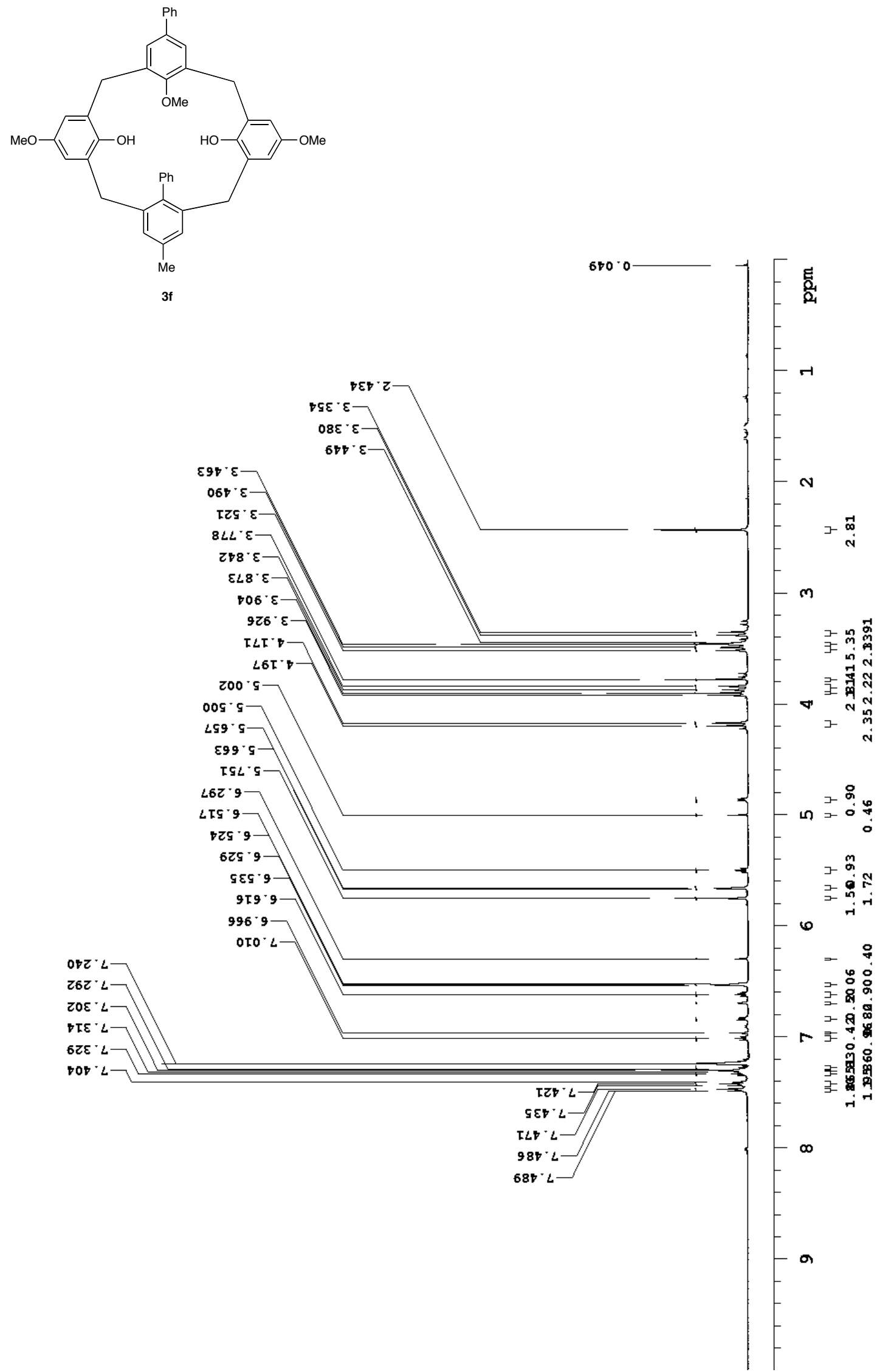

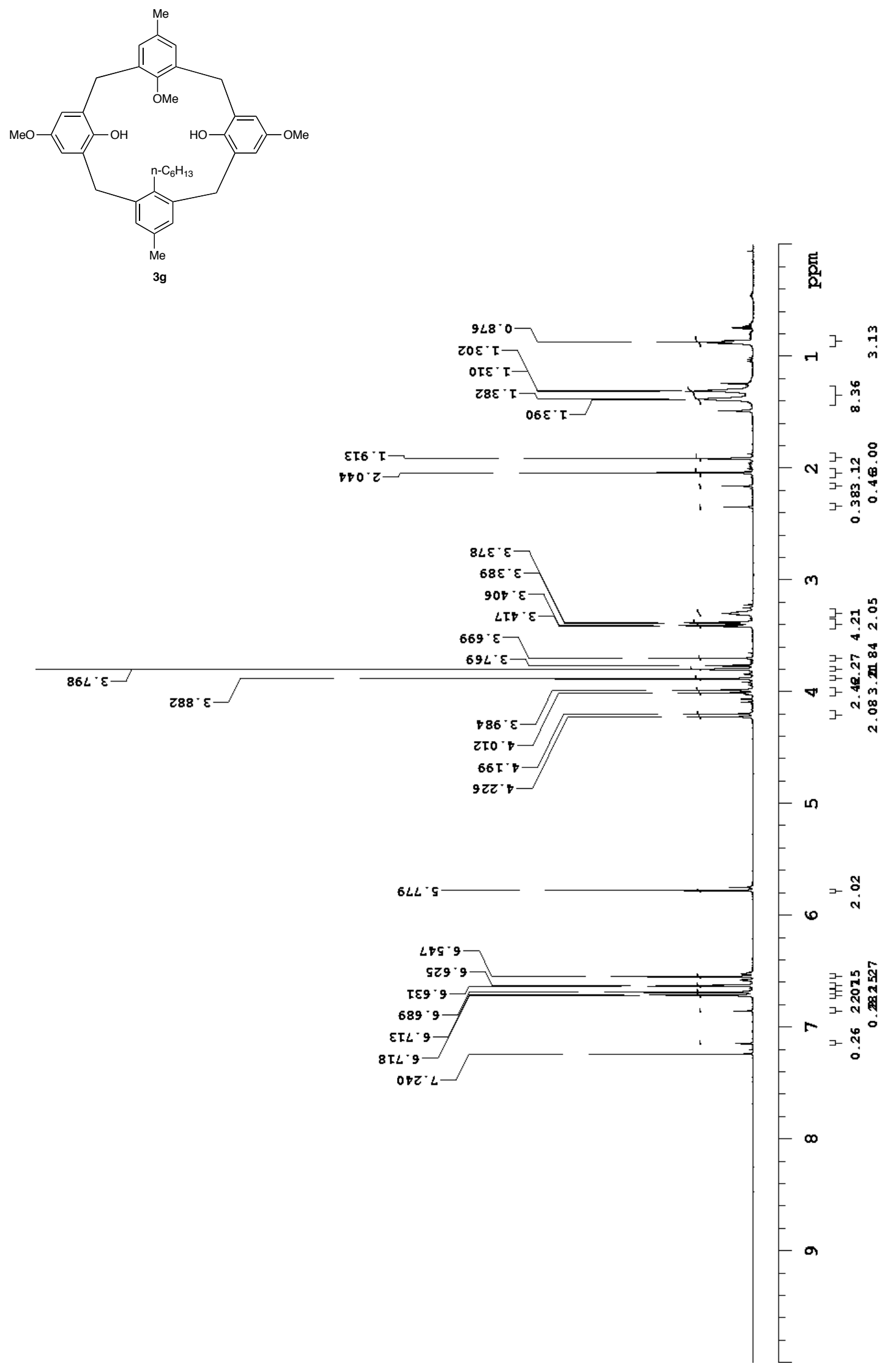

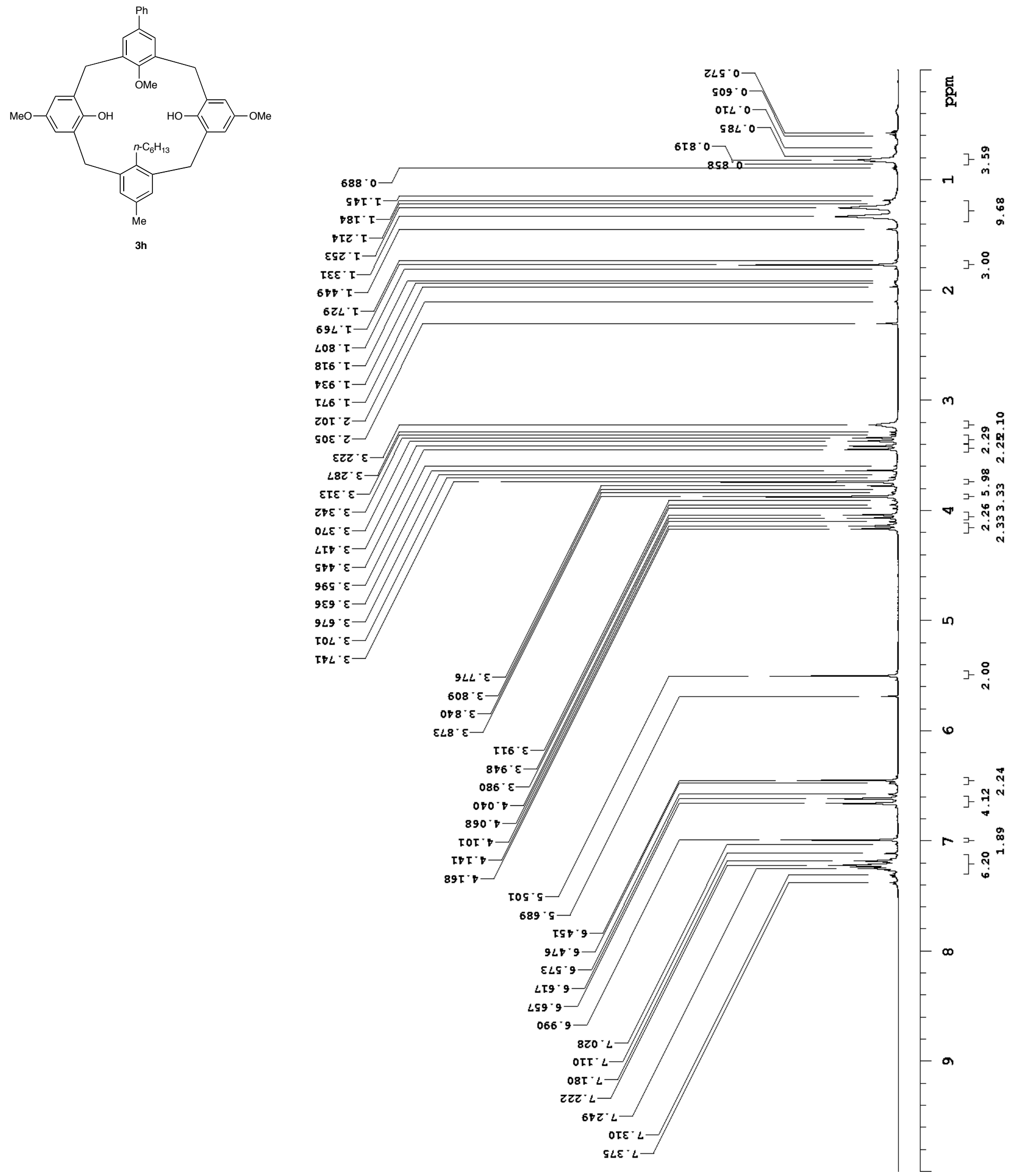


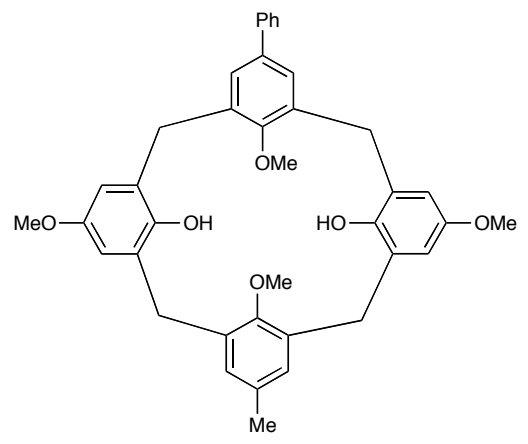

$3 \mathbf{i}$

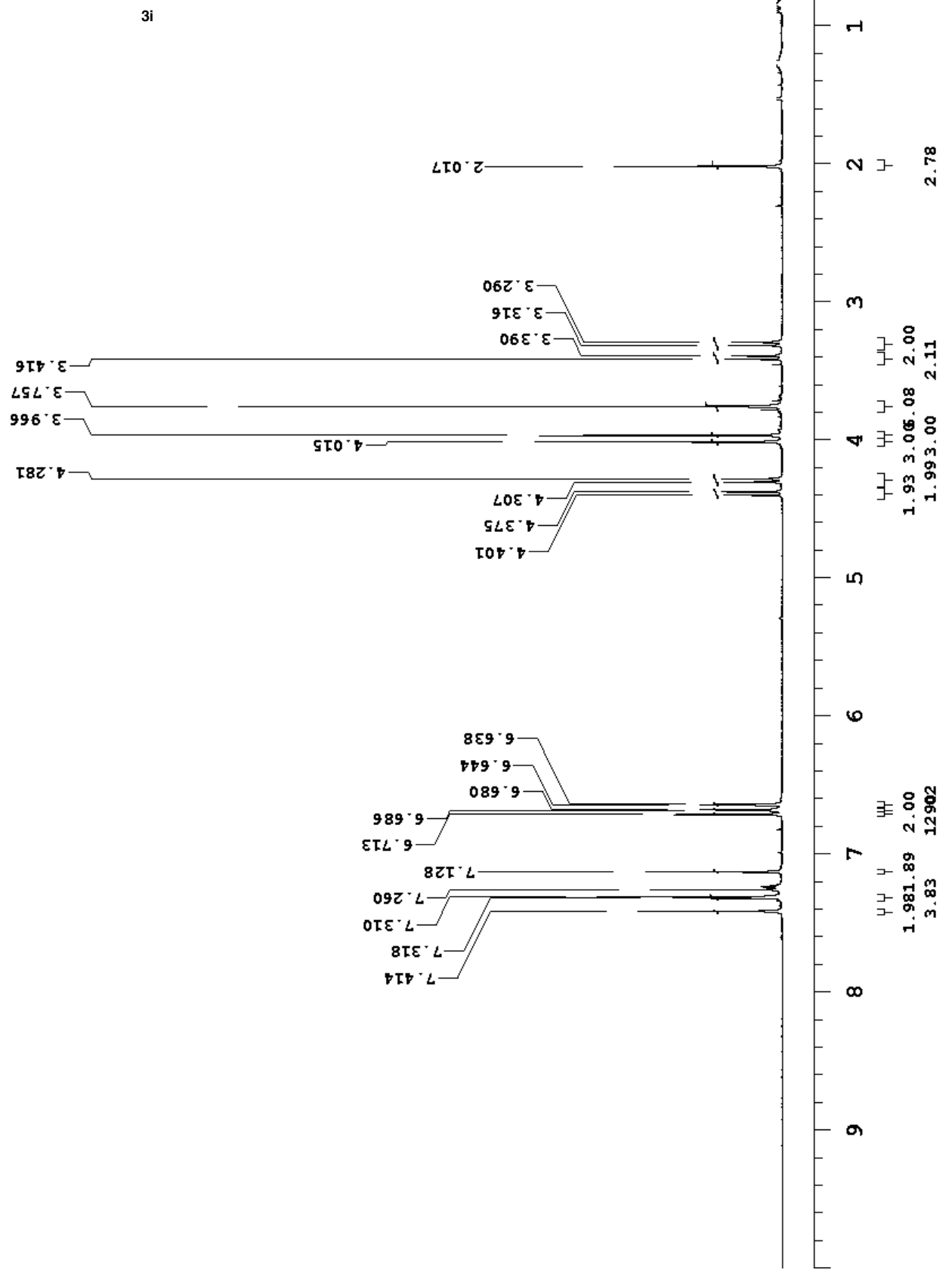

